Meta

Journal des traducteurs

Translators' Journal

\title{
Les prénoms dans la langue imagée
}

\section{Henri Van Hoof}

Volume 43, numéro 2, juin 1998

URI : https://id.erudit.org/iderudit/004531ar

DOI : https://doi.org/10.7202/004531ar

Aller au sommaire du numéro

Éditeur(s)

Les Presses de l'Université de Montréal

ISSN

0026-0452 (imprimé)

1492-1421 (numérique)

Découvrir la revue

Citer cet article

Van Hoof, H. (1998). Les prénoms dans la langue imagée. Meta, 43(2), 262-311.

https://doi.org/10.7202/004531ar

\section{Résumé de l'article}

Cet article est une étude de l'utilisation des prénoms par les langues française et anglaise. L'auteur étudie les la nature des prénoms tant bibliques et mythologiques qu'usuels, étrangers ou fictifs. Il traite de leurs formes puis des principales fonctions qui leurs sont attribuées. Il présente enfin un corpus bilingue de prénoms. 


\title{
ÉTUDES TERMINOLOGIQUES ET LINGUISTIQUES
}

\section{LES PRÉNOMS DANS LA LANGUE IMAGÉE}

\begin{abstract}
Résumé
Cet article est une étude de l'utilisation des prénoms par les langues française et anglaise. L'auteur étudie les la nature des prénoms tant bibliques et mythologiques qu'usuels, étrangers ou fictifs. Il traite de leurs formes puis des principales fonctions qui leurs sont attribuées. Il présente enfin un corpus bilingue de prénoms.
\end{abstract}

\begin{abstract}
This article studies the use of forenames in French and English. The autor studies the nature of Biblical, and mythological forenames as well as foreign and fictive ones. He also examines their forms and main functions. He illustrates with a bilingual corpus of fornames.
\end{abstract}

Déjà du temps que Berthe filait (jadis), le mari jaloux avait beau faire le Roland (menacer), cela n'empêchait pas sa Goton (femme facile) de le faire Jean (cocufier). Très juste, Auguste!

Dans la plupart des langues de culture, les prénoms jouent un rôle multiple et varié : (Fr) Anastasie, Jean-foutre, faire le Jacques (En) Dick, Jack-in-the-box, to look a proper Charlie (De) Otto, Miesepeter, frech wie Oskar sein (El) Maruja, santo Tomás, entrar como Pedro por su casa (N1) Jan, magere Hein, voor Piet Snot staan, etc. Non seulement sont-ils présents à tous les niveaux de langage, mais encore certains ont-ils réussi à se glisser dans les vocabulaires scientifiques et techniques : (Fr) tendon d'Achille (En) Achilles tendon (De) Achillessehne (El) tendón de Aquiles (N1) Achillespees; (Fr) herbe de SaintJean (En) St. John's-wort (De) Johanniskraut (El) hierba de San Juan (N1) Sint Janskruid; (Fr) coquille Saint-Jacques (En) Jacob's shell (De) Jakobsmuschel (N1) Jacobsschelp, etc.

Le plus grand nombre, cependant, se trouve dans la langue courante, conventionnelle ou non, alimentée en permanence par les créations sans cesse renouvelées de l'imaginaire populaire, créations qui vont et viennent au gré des siècles, disparaissant souvent très vite ou survivant parfois longtemps. Le texte en exergue est, de ce point de vue, artificiel dans la mesure où il se compose de locutions relevant d'époques différentes. La datation a donc son importance, même si l'on sait qu'elle ne peut jamais être qu'approximative. Nous nous sommes efforcés d'en tenir compte autant que possible dans cette étude qui se propose de comparer les utilisations de prénoms par les langues française et anglaise.

\section{DE LA NATURE DES PRÉNOMS}

Prénoms bibliques et mythologiques, prénoms usuels et étrangers, tout fait farine au moulin de la création linguistique.

\section{Prénoms bibliques et mythologiques}

Lorsqu'il s'agit de langues culturellement aussi proches que le français et l'anglais, les termes et expressions fondés sur des prénoms bibliques ou mythologiques se correspondent souvent littéralement dans les deux langues : talon d'Achille/heel of Achilles, le vieil Adam/the old Adam, le Benjamin de la famille/the Benjamin, fille d'Ève/daughter of 
Eve, un Hercule/a Hercules, pauvre comme Job/as poor as Job, baiser de Judas/Judas kiss, une Madeleine/a Magdalen, vieux comme Mathusalem/as old as Methuselah, une Pénélope/a Penelope, jugement de Salomon/justice of Solomon, etc., et cela même dans les domaines de spécialisation : figuier d'Adam/Adam's fig, sceau de Salomon/Solomon's seal, etc.

Il peut exister malgré tout quelque différence au niveau de la formulation : ami de Job/Job's comforter, poil de Judas/Judas-coloured hair, moïse/Moses basket, découvrir saint Pierre pour couvrir saint Paul/to borrow from Peter to pay Paul, un saint Thomas/a doubting Thomas, etc., sans qu'il y ait de modification de sens.

Il se peut aussi que la correspondance des prénoms soit incomplète : ne connaître ni d'Ève ni d'Adam/not to know from Adam, costume d'Adam/Adam and Eve's togs, Jésus, Marie, Joseph !/Jesus !, etc., inexistante par absence dans l'une des deux langues : pleurer comme une Madeleine/to cry one's eyes out, arbre de Moïse/Egyptian thorn, verge de Jacob/ yellow asphodel, millepertuis velu/Aaron's beard, être comme un coq en pâte/to feel as in Abraham's bosom, recevoir une bonne raclée/to pass through St. Peter's needle, etc., inexistante par substitution de prénom: étoile de David/Solomon's seal, faire le rébecca/to raise Cain, etc., voire illusoire car le bâton de Jacob (baguette de magicien) n'est pas le Jacob's staff (équerre d'arpenteur), le Jésus français (argot pour jeune homosexuel et pénis) n'a rien de commun avec le Jesus anglais (argot pour tripes, entrailles), l'arche de Noé (lieu de grand public ou de refuge) n'a rien à voire avec Noah's ark (pardessus), etc.

\section{Prénoms usuels}

Parmi les prénoms ordinaires, il en est de complètement désuets, qui ont donné naissance à des acceptions et à des locutions aujourd'hui obsolètes : (Fr) avoir le mal de saint Acaire, tout le Saint-Crépin, musique de saint Innocent, Frère Lubin, c'est un scrupule de saint Macaire, alleluia d'Othon, etc. (En) Abigail, to dine with St. Giles and the Earl of Murray, etc. Quelques-unes pourtant ont survécu à des niveaux de langue différents, soit moins académique soit technique: (Fr) eustache, fiacre, gervais, un vrai Nicaise, Philibert, etc. (En) Ignatius' bean, Prince Rupert's metal, etc.

Contrairement à ce que l'on observe pour les prénoms bibliques et mythologiques, les termes et expressions issus de prénoms ordinaires n'offrent que rarement une correspondance parfaite ou presque : Alphonse/Alphonse, Maître Jacques/handy Jack, Jacquet/ Jack, faire son Joseph/to play Joseph, laissez faire à Georges/let George do it, etc., sauf dans le cas de vocables scientifiques ou techniques : angélique sauvage/wild angelica (Bot.), feu Saint-Antoine/St. Antony's fire (Méd.), herbe de Saint-Christophe/herb Christopher (Bot.), Jean-doré/John Dory (Icht.), martin-sec/Martin dry (Bot.), herbe à Robert/ herb Robert (Bot.), etc.

Souvent les prénoms diffèrent d'une langue à l'autre: Anastasie/Dora, aller chez Bernard/to go to Sir Harry, être bien Claude/to be a proper Charlie, Julie/Jane, Jill, faire sa Julie/to talk Miss Nancy, avoir Marianne dans l'œil/to be as blind as Chloe, Martin/ Dicky, Neddy, il y a plus d'un âne qui s'appelle Martin/there are more Jacks than one at the fair, Mohamed/Betsy, frayer avec Pierre et Paul/to be hail-fellow-well-met with Dick and Harry, Pierre, Paul et Jacques/Tom, Dick and Harry, etc., et cela est vrai aussi pour certaines appellations populaires des domaines botanique et autres : pied d'Alexandre/Peter's wort, petite angélique/herb Gerard, jeannette/poet's narcissus, julienne/Jack-by-thehedge, véronique/Paul's betony, etc.

Plus fréquemment encore, le prénom n'existe pas dans l'une ou l'autre langue. Ainsi, l'anglais n'en a pas pour Bernard/the bottom, coiffer sainte Catherine/to remain unmarried at 25, faire Jean/to cuckold, cravate à Gustave/sanitary towel, mathurins/teeth, roberts/ 
female breasts, etc.; le français n'en a pas pour Benedict/nouveau marié, to turn Catherine wheels/faire la roue, dumb Dora/femme stupide, to wiggle one's fanny/se dépêcher, Gabriel/trompettiste, to cuff Jonas/marcher les genoux en dedans, lusty Lawrence/coureur de jupons, to give s.o. a Roland for an Oliver/rendre à qqn la monnaie de sa pièce, etc. Et cela se vérifie au niveau des vocabulaires spécialisés : augustine/silk cloth (Tex.), GrosGuillaume/variety of table grapes (Bot.), joseph/filter-paper (Chim.), lambert/saury (Icht.), marceline/natural manganese oxide (Chim.), martin rose/rose starling (Orn.), Gros Michel/plantain banana (Bot.), philémon/a genus of passerines (Orn.), etc.; à l'inverse, Barnaby thistle/centaurée du solstice (Bot.), Catherine wheel/rosace (Arch.), Harry-bird/ grand puffin (Orn.), Jack curlew/petit courlis (Orn.), creeping jenny/lysimaque nummulaire (Bot.), Jill/furet femelle (Zool.), John-go-to-bed-at-noon/salsifis sauvage (Bot.), herb Louisa/verveine citronnelle (Bot.), St. Peter's fish/aiglefin (Icht.), Tom-noddy/macareux (Orn.), etc.

\section{Prénoms étrangers}

Le français, comme l'anglais, adopte à l'occasion l'un ou l'autre prénom étranger. Ainsi, en français, dans : allons-y, Alonzo !, Carlos, Fridolin, Fritz, balançoire à Mickey, Mohamed, chanter Ramona à qqn, oncle Sam, Teddy, Tommy; en anglais, dans : Antonio, Fritz, to be on the fritz, Hans, Hans Carvel's ring, Heinie, Ivan, Tony, etc.

\section{Prénoms fictifs}

Par prénoms fictifs, il faut entendre ici des prénoms issus de jeux de mots; ce sont des prénoms qui, certes, existent, mais dont l'emploi est suggéré par une quelconque attraction paronymique, analogie de sens ou de son, et obtenus par une déformation délibérée ou inconsciente.

En français, le prénom casimir, pour désigner un tissu sergé croisé, n'est que la transcription fautive de l'anglais kerseymere, lui-même une corruption de cassimere, cashmere; dans l'exclamation tu es un Charles! (tu racontes des histoires !), le prénom n'est que l'abréviation de charlatan; dans café de sainte Claire, l'allusion à l'adjectif clair est évidente; faire Cléopâtre (faire une fellation) n'aurait aucun rapport avec la reine d'Égypte mais cacherait le jeu de mots «faire une clé au pâtre»; dédé, au sens de postérieur provient du redoublement de dé, anus; diane, dans les acceptions de chant matinal, sonnerie de réveil, papillon diurne, vient de dia, lui-même dérivé du latin dies; dominique, désignant le coffre de paie d'un navire, est inspiré de l'inscription Domine salvum fac regem qui figurait sur les écus; Marie-Jeanne est un phonétisme plaisant de marijuana; dans vieux comme Mathieu-salé, l'attraction de Mathusalem est manifeste; faire la rue Michel (faire le compte, suffire) est le fruit d'un jeu de mots sur la rue Michel-Leconte à Paris; dans chanter Ramona à qqn (réprimander), le prénom est engendré par le verbe de même sens ramoner; tout comme il l'est par l'ancien verbe rebéquer dans faire du rébecca (protester, regimber); quant à la boisson roméo, elle est le résultat physique et phonétique du mélange rhum et eau.

De même, en anglais, Amy-John (lesbienne) est une transcription facétieuse d'amazon, tout comme Maggie Ann, Margery Jane et Mary Jane le sont de margarine, et tout comme Mary Ann l'est de marihuana. L'exclamation san fairy Ann! (peu importe !) est la traduction phonétique du français ça ne fait rien, tout comme billy-doo l'est de billet doux et John Darm (policier) de gendarme. C'est par le même procédé que Bill Harris en est venu à remplacer un terme médical, la bilharziose. Dans benjamin-bush, le prénom n'est qu'une déformation de benjoin; de même, le johnny-cake n'est qu'une corruption de journey-cake. Dans Jimmy Grant, pour immigrant, le décalque phonétique et graphique est quasi total; dans Mona pour moaner (geignarde), il n'est que phonétique. Lizzie est l'apo- 
cope suffixée de Lisbon wine; Louie, travailleur migrant aux États-Unis, est l'abréviation de Louisiana, l'État dont ils étaient originaires dans les premiers temps; Ted, au sens de soldat allemand, est l'apocope de l'italien Tedesco. Peggy (unijambiste) est inspiré de peg, pilon. Rufus (sexe de la femme) subit l'attraction de rufous, roussâtre, d'après la couleur des poils pubiens. William pear est une erreur pour Williams' Bon-Chrétien, d'après le nom du premier distributeur de cette variété de poire.

Dans la catégorie des prénoms fictifs (ou, si l'on préfère, artificiels ou arbitraires), il convient de ranger aussi ceux générés par des procédés argotiques, comme le verlan en français ou le rhyming slang (argot rimé) en anglais. Le verlan, qui consiste à inverser l'ordre des syllabes ou des lettres d'un mot, a ainsi produit luc (cul) et bon Jean (jambon). Dans le rhyming slang (RS), qui remplace un mot par un groupe de deux ou plusieurs autres dont le dernier rime avec lui, la rime choisie est souvent un prénom : Cain and Abel (table), Adam and Eve (to believe), Anna Maria (fire), Big Ben (ten (pounds)), King Dick (brick), Uncle Dick (prick, i.e. penis), Bob, Harry and Dick (sick), Levy and Frank (whank, i.e. masturbation), Uncle Fred (bread), Jack an Jill (bill ou hill), Kate and Sidney (steak-and-kidney (pudding)), mad Mick (pick ou prick), Pat and Mick (lick, i.e. thrashing), clever Mike et Pat and Mike (bike), Uncle Ned (bed), Uncle Willie (silly), etc.

\section{DE LA FORME DES PRÉNOMS}

Les multiples exemples cités jusqu'ici permettent déjà de constater que les prénoms sont utilisés sous différentes formes : tels quels ou abrégés, de manière autonome, dans des composés, dans des locutions.

\section{Les prénoms autonomes}

Nombreux sont les prénoms qui, employés seuls, ont été gratifiés d'un sens imagé. Parmi les prénoms bibliques et mythologiques, le français offre Achille, David, Hercule, jéroboam, Jésus, Joseph, Judas, Mathieu, moïse, Narcisse, Nestor, Pénélope; l'anglais possède Abraham, David, Gabriel, Hector, Hercules, Jacob, Jeremy, jeroboam, Jesus, Job, Jonas, Moses, Narcissus, Nestor, Penelope, etc. Leur sens imagé peut varier d'une langue à l'autre : le David français (argot pour crochet de cambrioleur ou casquette, selon l'époque) n'est pas le David anglais (déclaration sous serment), le moïse français (berceaucorbeille) n'est pas le Moses anglais (homme humble et soumis ou prêteur juif), etc.

De pareils faux amis seraient impossibles en ce qui concerne les prénoms désuets, où le français ne présente guère que les Anastasie, eusèbe, eustache, fiacre, gervais, Lubin et autres Robin, qui ne risquent pas d'être confondus avec les Abigail ou autres Rupert anglais.

Le cas des prénoms ordinaires toujours usités est plus complexe. Ils sont légion tant en français qu'en anglais et bon nombre d'entre eux - Agnès, Arthur, augustine, Basile, Bernard, Bertrand, Gustave, Julien, lambert, Louis, marceline, Philippe, Simon, etc., d'une part, Andrew, Archibald, Benedict, Cecil, Clarence, Ethel, George, Herman, Jonathan, Lawrence, Melvin, Oliver, Richard, Wilfred, etc., d'autre part - diffèrent totalement quant à l'aspect et aux notions qu'ils recouvrent; ils ont donc leurs lacunes respectives dans l'une et l'autre langue. D'autres, au contraire, diffèrent d'une langue à l'autre pour une même acception : Baptiste, Claude, Jacques, etc./Sam, Tony pour nigaud, Caroline/Charley, Molly, Oscar, Willie pour homosexuel, Charlotte, Félix, Gaspard, etc./Dick, Jack, Peter, Roger, Timothy, etc. pour pénis, Gaspard, Monique/Fanny, Rufus pour le sexe de la femme, Jacques/Peter pour coffre-fort, Marcel/John pour ami de cœur, Jacques, Thomas/ Jemima, Jordan pour pot de chambre, Alphonse, Jules, Prosper, etc./Alphonse, Louis, etc. pour proxénète, etc. 
On note ainsi qu'il peut exister des correspondances partielles pour un même prénom dans les deux langues. C'est aussi le cas pour charlotte, qui désigne le plat d'entremets en anglais comme en français, mais dont les autres sens français ne sont pas traduits en anglais, si ce n'est celui de pince-monseigneur par les diminutifs jenny et jimmy. C'est aussi le cas pour Madeleine, qui désigne la pécheresse repentie et la variété de poire dans les deux langues mais dont les sens de biscuit de Savoie et de cépage ne sont pas rendus en anglais.

Enfin, les prénoms ordinaires connaissent eux aussi leurs correspondances illusoires : la Gertrude française (argot de musiciens pour contrebasse) n'est pas la Gertrude anglo-saxonne (grue de prise de vues dans l'argot du cinéma), le guillaume français (rabot) n'est pas le William (thé léger) des Britanniques, le louis (pièce de vingt francs) n'est pas le Louis (proxénète) londonien, le robert (sein) n'est pas le Robert (policier ou shilling), le Thomas (vase de nuit) n'est pas le Thomas (pénis, serveur ou valet) d'OutreManche.

Les formes diminutives de prénoms, qu'elles soient obtenues par aphérèse avec ou sans déformation (Colas, gusse; Fred, Tony), par apocope (Josse; Alec, Ben, Kate), par contraction (Fanchon, Goton; Bess, Jeff, Meg) ou par suffixation (Charlot, Jacquet, Jeannette, Julot; Archie, Johnny, Maggie), sont également très sollicitées par la langue imagée, particulièrement en anglais où elles sont deux fois plus nombreuses qu'en français. Le français, en revanche, se distingue par un procédé hypocoristique supplémentaire, la reduplication : dédé, Dudule, fifine, gugusse, jeanjean, nénette, Popol, etc.

\section{Prénoms et mots composés}

Sous leur forme régulière ou diminutive, les prénoms servent à créer des mots composés de nature diverse. Le procédé le plus classique, qui consiste à les relier à d'autres mots par des traits d'union, a donné naissance en français à Gros-Guillaume, Jean-doré, Jean-foutre, Jean-nu-tête, Petit-Jean, dame-jeanne, larme-de-Job, Gros-Léon, Louisquinze, marie-galante, marie-pisse-trois-gouttes, marie-salope, martin-sec, fesse-mathieu, Gros-René, etc., pour les prénoms réguliers, lili-pioncette pour les diminutifs; en anglais : merry-andrew, benjamin-bush, Harry-bird, John-a-dreams, John-go-to-bed-at-noon, tarry-John, Mary-lily, Robin-run-in-the-hedge, tickle-Thomas, sweet-William d'une part, et, preuve de l'abondance des diminutifs dans cette langue : billy-goat, fore-and-aft Fanny, Jack-by-the-hedge, Jack-in-the box, jumping-jack, Jerry-go-nimble, Joe-pye-weed, Johnny-come-lately, Johnny-on-the-spot, molly-coddle, nanny-goat, Tom-noddy, tommyrot, pee-Willy, etc., d'autre part. Il n'est pas rare que les traits d'union soient omis, comme dans Jean qui de tout se mêle, Jean qui ne peut, etc. en français, what Harry gave Doll, Jack in office, Jack of all trades, dumbjohn, Paddyland, tomfool, etc. en anglais.

Un deuxième type de composés réunit le prénom à d'autres mots par une préposition. Ainsi, en français, talon d'Achille, fourchette d'Adam, café de sainte Claire, prix de Diane, fille d'Ève, cravate à Gustave, danse de Saint-Guy, échelle de Jacob, bâton de Saint-Jacques, Jean de la vigne, pain de saint Jean, femme à Job, Julie du Brésil, fleur de Marie, été de la Saint-Martin, arbre de Moüse, herbe à Robert, sceau de Salomon, etc. pour ce qui concerne les prénoms réguliers, bascule à Charlot, poisse à Dudule, coiffure à la Ninon, boîte à Perrette, imperméable à Popol, etc. pour les diminutifs. L'anglais, par son cas possessif présent ou non dans la graphie, crée Aaron's rod, Adam's ale, Anthony's fire, Barnaby thistle, Catherine wheel, herb Christopher, Dorothy bag, bean of St. Ignatius, Jacob's sword, Jesus's eyes, Job's news, Joseph's coat, Lot's wife, herb Louisa, Moses basket, Oliver's skull, Peter's fish, Robin's plantain, Samson's post, etc. avec des prénoms réguliers, bobby's job, Davy's locker, Dick's hatband, Maggie's drawers, Molly's hole, Paddy's grapes, etc. avec des diminutifs. 
Dans un troisième type de composés, la juxtaposition d'un prénom et d'un nom ou d'un adjectif mène à des syntagmes de qualification où le prénom joue le rôle soit du déterminant soit du déterminé. Le français aligne ainsi : le nouvel Adam, Madame Arthur, grosse Bertha, Jacques Bonhomme, Maître Jacques, bon Jean, double Jean, p'tit Jésus, affreux jojo, Messire Luc, ours Martin, Michel bonhomme, gros Michel, plaisant Robin, Roger Bontemps, un saint Thomas, etc.; l'anglais possède the old Adam, big Bertha, brown George, hopping Giles, Calamity Jane, plain Jane, homely Joan, John dogface, John Law, square John, fancy Joseph, Black Maria, little Mary, Paul Pry, old Roger, simple Simon, man Thomas, etc. et, preuve nouvelle de son fréquent recours aux diminutifs : handy Andy, smart Alec, anytime Annie, brown Bess, puffing Billy, silly Billy, old blind Bob, cheap Charley, fancy Dan, old Davy, clever Dick, dumb Dora, Uncle Fred, alibi Ike, Jack Sprat, Jack Tar, flash Jack, country Jake, creeping jenny, dismal Jimmy, cloudy Joe, holy Joe, Johnny Armstrong, jingling johnnie, skinny Lizzie, long Meg, nice Nellie, old Nick, sneaky Pete, Uncle Sam, simple Sammy, Sister Susie, Long Tom, weary Willie, etc.

Parmi ces derniers exemples, il en est qui relèvent de procédés de création linguistique très prisés de l'anglais, à savoir l'allitération (anytime Annie, brown Bess, cheap Charley, dumb Dora, jingling johnnie, nice Nellie, Paul Pry, simple Sammy, sister Susie, weary Willie) - rare en français (Gautier et Garguille, Gros-Guillaume) - , les mots rimés (handy Andy, silly Billy, plain Jane, skinny Lizzie) et l'argot rimé (Uncle Dick, Uncle Fred). L'allitération a encore produit Burlington Bertie, Dicky Diddle, fore-and-aft Fanny, John-and-Joan, jumping-jack, Kate Karney, lazy Lawrence, Percy-pants, pretty Poll, Richard Roe, Sammy Soft, silent Susan, Tom Tyler, etc. D'autres mots rimés sont Abe's cabe, Anne's fan, Fanny Nanny, Georgy-Porgy, screwy Louie, Roger the Lodger, even-Steven, silly Willy, etc. Quand à l'argot rimé, il accuse une prédilection marquée pour les prénoms comme l'un de ses composants. Outre les exemples cités précédemment (cf. Prénoms fictifs), où le prénom était porteur de la rime, il y a la grande majorité des cas où il ne fait qu'accompagner arbitrairement (mais parfois encore par attraction allitérative ou rimée) un patronyme imaginaire qui rime avec le mot remplacé : Andy Cain (rain), Annie Laurie (lorry), Billy Button (mutton), Charley Howard (coward), Davy Large (barge), Dicky Dirt (shirt), Fanny Blair (hair), Harry Randall (candle), Henry Nash (cash), Jack Horner (corner), Jane Shore (whore), Jenny Lea (tea), Jim Mace (face), Joe Blake (cake), Joe Rocks (socks), John Hall (alcohol), Johnnie Rutter (butter), Mary Blane (train), Mick O'Dwyer (fire), Ned Kelly (belly), Nellie Bligh (eye), Oliver Twist (fist), Paddy Rammer (hammer), Polly Flinder (window), Sandy McNab (cab), Thomas Tilling (shilling), Tom Thacker (tobacco), Tom Tripe (pipe), Tom Tug (bed-bug), Tommy Roller (collar), Willy Lee (flea), etc. Rimées le plus souvent avec des mots du langage courant, ces formations peuvent l'être aussi avec des mots du répertoire argotique, comme dans Charley Brady (cady; chapeau), Charley Ronce (ponce; proxénète), Ellen Terry (jerry; pot de chambre), Harry Wragg (fag; cigarette), Jerry Riddle (to piddle; uriner), John Hop (cop; policier), Maria Monk (spunk; courage ou sperme), Oliver Cromwell (to tumble; comprendre), Tommy Dodd (sod; homosexuel), Tommy Rollocks (ballocks; testicules), etc.

\section{Prénoms et locutions}

Les prénoms, quelle que soit leur forme, entrent dans la composition d'une multitude de locutions de la langue imagée : locutions verbales, adjectivales, adverbiales, interjectives, ainsi que dans celle de proverbes et de dictons.

Dans la famille des locutions verbales, très nombreuses aux différents niveaux de langue, on trouve en français : avoir son Arnaud, appeler Arthur, aller chez Bernard, coiffer sainte Catherine, amuser Charlot, baiser Fanny, avaler le Gaspard, faire le Jacques, faire son petit saint Jean, faire son jojo, faire sa Joséphine, se faire appeler Jules, lâcher 
une louise, pleurer comme une Madeleine, avoir la Marianne dans l'œil, fesser Mathieu, étrangler Popol, chanter Ramona à qqn, faire sa rébecca, faire le Roland, voir Sophie, etc.; en anglais : to cuff Anthony, to hug Brown Bess, to raise Cain, to introduce Charley, to take one's Daniel, to take one's Davy on it, to park one's fanny, to go to Sir Harry, to dine with Duke Humphrey, to get Jack in the orchard, to knock the Jesus out of s.o., to crack a $J u d y$ 's teapot, to have Lawrence on one's back, to stand Moses, to talk Miss Nancy, to give s.o. a Roland for an Oliver, to go through St. Peter's needle, to get the Richard, to stand Sam, etc. Certaines de ces locutions sont construites avec l'appui d'une rime intérieure, par ex. dans être comme Jules, incrédule et, en anglais, to sham Abraham, to have the cheek of old Nick.

Au rayon des locutions adjectivales, le français aligne en costume d'Adam, tranquille comme Baptiste, de la Saint-Jean, beau comme un Jésus en cire, en Jésus, pauvre comme $J o b$, léger comme l'oiseau saint Luc, du même saint-Martin, vieux comme Mathusalem, hardi comme un saint Pierre, gai comme un pierrot, etc.; l'anglais aussi possède as poor as $J o b$ et as old as Methuselah, mais en outre as old as Adam, as blind as Chloe, Dolly Cotton, as drunk as David's sow, on one's Jack, on one's Darby and Joan, as knowing as Kate Mullet, as happy as Larry, as salt as Lot's wife backbone, as long as Meg of Westminster, as Irish as Paddy's pig, on one's Pat, as fat as Sir Roger, etc. Ici encore, l'anglais recourt parfois à la rime intérieure, comme dans as happy as Larry, à l'allitération qui lui est chère, comme dans as meek as Moses, voire à l'argot rimé comme dans on one's Darby and Joan (alone) et on one's Pat (abrégé de on one's Pat Malone, même sens).

Les locutions adverbiales sont représentées en français par à l'hasard, Balthazar, du temps que Berthe filait, au temps de Mathusalem, comme un fiacre, du temps du roi Guillemot, etc.; l'anglais par since Adam was a boy, like billy-o, in the days of Queen Dick, on St. Geoffrey's day, since Hector was a pup, before you can say Jack Robinson, since Nellie had her operation, etc., faisant parfois encore appel à l'allitération comme dans by guess and by Godfrey ou since Pontius was a pilot (avec un jeu de mot évident).

Les locutions interjectives sont celles peut-être qui exploitent le plus l'artifice de la rime intérieure : tu vas me le payer, Aglaé !, ça glisse, Alice !, fonce, Alphonse !, ça colle, Anatole !, tout juste, Auguste !, à l'aise, Blaise !, tu parles, Charles !, à la tienne, Étienne !, recule, Hercule !, tu dis vrai, Jacquet !, relaxe, Max !, cool, Raoul!, en français; whoa, Emma!, have a go, Joe !, believe me, Steve !, en anglais. Sans cet artifice, le français connaît encore t'as le bonjour d'Alfred !, adieu, Berthe !, tu es un Charles !, elle est revenue, Denise !, vous êtes orfèvre, monsieur Josse !, vas-y, Léon !, pas de ça, Lisette !, Nicolas, j't'embrouille !, c'est la toile de Pénélope !, en voiture, Simone !; l'anglais, de même, est riche en plus de Queen Anne is dead !, Archibald, certainly not !, all Betty!, Bob's your uncle !, Charley Brown !, my aunt Fanny !, by George !, not for Joe !, outside, Liza !, for the love of Mike !, holy Moses !, ask my Nancy!, not on your Nellie Duff !, upon my Sam !, come on, Steve !, etc., parfois avec une forme allitérative telle Good Godfrey!, comme d'ailleurs en français allons-y, Alonzo ! ou c'est Perrette et le pot au lait !

Rime et allitération sont des procédés qu'affectionnent aussi les proverbes et les dictons. Ainsi, en français : qui croit guiller Guillot, Guillot le guille; il fait comme le chien de Jean de Nivelle, qui s'enfuit quand on l'appelle; c'est aujourd'hui la Saint-Lambert, qui quitte sa place la perd; à la Sainte-Luce, les jours croissent d'un saut de puce; s'il pleut le jour de la Saint-Médard, il pleut quarante jours plus tard; en anglais : for every Jack there is somewhere a Jill that can do with poor Jack about as she will. À ces formulations mnémoniques, on peut ajouter c'est sainte Geneviève et saint Marceau, à chaque porc vient la Saint-Martin, il y a plus d'un âne (à la foire) qui s'appelle Martin, toujours souvient à Robin de ses flûtes, c'est saint Roch et son chien, pour le français; et, pour l'anglais, there's no leaping from Dahlila's lap into Abraham's bosom, one law for Dick and another for 
Harry, a Jack of all trades is a master of none, all work and no play makes Jack a dull boy, every Jack has his Jill, if Jack's in love he's no judge of Jill's beauty, there are more Jacks than one at the fair, etc.

\section{DE LA FONCTION DES PRÉNOMS}

Parmi les principales fonctions réservées aux prénoms, tant en anglais qu'en français, il y a la personnification de parties du corps, d'animaux et d'objets, la caractérisation de types humains, de nationalités et d'activités ou de professions, la formation de termes scientifiques ou, plutôt, de leurs doublets populaires, la fonction explétive.

\section{Personnification}

Certaines parties du corps ont toujours inspiré l'invention linguistique et la personnification du sexe de l'homme ou de la femme, en particulier, s'exprime dans une riche moisson : cheville d'Adam, Charles le Chauve, Charlotte, Félix, Ferdinand, Gaspard, Jacquot, Pascal, Philibert, Philidor, Popaul/Abraham, old Adam, blind Bob, little Davy, dick, jack, Jack Robinson, Jenny Donnelly, Jezebel, John Thomas, johnnie, mad Mick, Peter, Roger, Rupert, man Thomas, Timothy, Long Tom, Tommy, little Willie pour le premier, Gaspard, mimi, monique, boîte à Popaul/Fanny, Hans Carvel's ring, Jack Nasty-Face, Jack Straw's castle, Aunt Maria, Mary Jane, Molly's hole, mother of St. Patrick, Rufus, tickleThomas pour le second. Le postérieur est appelé Bernard, dédé, fiacre, pétrus/Fanny; les seins féminins deviennent des roberts/Lewis and Witties; la main est la fourchette, le mouchoir ou le peigne d'Adam/Mary Ann. Le français et l'anglais ne personnifient pas toujours les mêmes parties du corps : le français nomme jacques, jacquots les mollets, luc l'anus, fleur de Marie l'hymen, mathurins les dents, nénette le cerveau, épée de Samson les mâchoires; pour l'anglais, les cheveux deviennent Fanny Blair, le nez Harry James, le visage Jim Mace, l'estomac little Mary, le ventre Ned Kelly, l'œil Nellie Bligh, les testicules Tommy Rollocks.

Parmi les animaux, il en est que la langue imagée a personnifié de longue date, tels l'âne Martin/dicky, jackass, Ned(dy) ou le perroquet Jacquot/Poll(y), pretty Poll, tels encore en français le rat Gaspard, l'écureuil Jacquet, la pie Margot, le moineau pierrot, etc. ou en anglais le bouc billy, le renard Charley, l'ânesse jenny, le lapin Molly cotton-tail, la chèvre nanny, le matou tom-cat. Il en est d'autres dont l'appellation relève davantage des vocabulaires spécialisés, tels l'oiseau marin guillemot, le coléoptère géant hercule, le gallinacé pénélope, le pigeon victoria, etc., que l'anglais connaît généralement sous les mêmes noms.

Les objets personnifiés sont d'une infinie variété, allant du couteau de poche achille, eustache, jésus/jack-knife au vase de nuit carlos, jacques, thomas/Ellen Terry, jemima, jerry, jordan, Oliver's skull, de l'argent carlos/jack au coffre-fort jacques/Pete(r), de la pince-monseigneur charlotte, jacques, jacquot/jemmy, jenny, Jim Crow, Jimmy au rossignol David, Joséphine/Bess, Betty, Kate, du grand manteau d'homme lisettelbenjamin, benny, Noah's ark au soleil Mahomet, Mohamed/Betsy, de la marijuana marie-jeannel Mary Ann à la cocaïne Julie du Brésil/Cecil, Charley Coke, etc. Le français baptise encore la nuit bernarde, la baïonnette Charlemagne, Rosalie, la guillotine bascule à Charlot, la serviette hygiénique fifine, cravate à Gustave, la morphine lili-pioncette, le préservatif imperméable à Popaul; en anglais, la table devient Cain and Abel, la margarine Maggie Ann, Margery Jane, Mary Jane, l'eau Adam's ale, la bière Charley Freer, le pain Uncle Fred, l'assiette Harry Tate, le café Joe, la vieille voiture tin Lizzie, la voiture cellulaire Black Maria, la pomme de terre mickey, la lune Oliver, etc. 


\section{Caractérisation}

Le nigaud, le poltron, le bravache, le vantard et bien d'autres types humains ont de tous temps suscité la dérision, dérision qui s'est traduite par des sobriquets divers. Ainsi, Baptiste, Claude, Colas, Jacques, Janot, Jean(not), Mimile, Nicaise, Nicodème, Nicolas, Pierrot/silly Billy, dumbjohn, Sam, Sammy Soft, simple Simon, Tom Cony, Tom Fool, Tony, silly Willie pour le benêt; Charlot, enfant de Marie/Alec pour le naï; Jean/Charley Howard, Ethel pour le poltron; marie-salope/dolly pour la souillon; Mme Arthur, Caroline, Jésus/Mary Ann, Charley, John-and-Joan, (Miss) Molly, Nancy (boy), Oscar, Tommy Dodd, Willie pour l'homosexuel, affreux jojo/Jack Nasty-Face pour un individu peu recommandable, etc. Le français possède en outre des surnoms pour la petite oie blanche, Agnès, le coquin, Bertrand, la bonne fille pas trop farouche, Fanchon, le cocu, Jean, bon Jean, le bon à rien, Jean-foutre, la femme bavarde, Margot, le joyeux drille, Roger Bontemps, etc.; de même, l'anglais nomme Amy-John la lesbienne, Jack Brag le vantard, Jack Sauce l'insolent, Jake, honest John l'honnête homme, plain Jane le laideron, dismal Jimmy le rabat-joie, Jonah, Jonas le guignard, lusty Lawrence le coureur de jupons, red Mike le misogyne, holy Joe, nice Nellie le pudibond, Paul Pry le curieux, Peter Grievous le pessimiste, lazy Lawrence, weary Willie le paresseux, etc.

Le prénom sert aussi de signe d'identité pour une nation ou ses ressortissants : Fridolin, Fritz/Freddy, Fritz, Hans, Heinie, Jerry désignent l'Allemand, Yvan/Ivan le Russe, John Bull/John Bull personnifie la nation britannique ou l'Anglais type, oncle Sam/Brother Jonathan, Uncle Sam les États-Unis ou les Américains, etc. Le français use encore de Michel Bonhomme pour le bourgeois allemand, de Mohamed pour l'Arabe; l'anglais de Antonio, Tony pour l'Italien ou le Portugais, de Abie, clipped Dick, Ike pour le Juif, de Jock pour l'Écossais, de Mick, Mickey, Paddy, Pat pour l'Irlandais.

Nombreuses sont les activités ou professions, honorables ou non, qui ont pris pied dans la langue imagée par le truchement de prénoms. À côté du paysan Jacques Bonhomme, Petit-Jean, Pierrot/Bill Shears, hill-billy, Johnny Whop-Straw, Harry, Jasper, Reuben, Rube et du marin mathurin/Jack, Jack Nasty-Face, Jack Tar, tarry-John, à côté du domestique Jacquet/Jack, Thomas et de l'homme à tout faire Maître Jacques, Jean faittout/Jack of all trades, à côté du voleur Charles/John Family et du bourreau Anatole, Charlot/Jack Ketch, il y a l'usurier fesse-Mathieu/Ike, Moses, le proxénète Alphonse, Carlos, la poisse à Dudule, Jules, Julot, Prosper/Alphonse, Charley (Ronce), fancy Joseph, Louis, Tommy, la prostituée Julie, marie-salope/anytime Annie, brown Bess, cousin Betty, fore-and-aft Fanny, Flossie, easy Jane, Jane Shore, (Miss) Molly, Tom. Le français a personnifié en outre le président d'assises Anatole, Gros-Léon, le petit ramoneur Ramona, le serviteur de confiance Gros-René, etc.; l'anglais a fait de même pour le tailleur Abie, Jack Snip, le juge Barnaby Rudge, l'ouvrier agricole Billy Turniptop, le policier bobby, dick, jack, Robert, le trompettiste Gabriel, Joe Blow, le météorologue cloudy Joe, le prêtre ou aumônier holy Joe, le travailleur manuel Johnny Armstrong, etc.

\section{Formation de termes scientifiques}

Si quelques locutions du genre tendon d'Achille/Achilles tendon, pomme d'Adam/ Adam's apple en médecine, arbre de Diane/arbor Dianae, métal du prince Robert/Prince Rupert's metal en chimie, ont parfois acquis droit de cité dans les vocabulaires spécialisés, c'est principalement dans la création de doublets populaires pour des termes plus savants que les prénoms ont trouvé leur plus large application. Dans le cas du français et de l'anglais, ce sont généralement les mêmes, comme en médecine feu Saint-Antoine/(St.) Anthony's fire, danse de Saint-Guy/St. Guy's dance (mais aussi St. Anthony's, St. John's et St. Vitus' dance), en botanique figuier d'Adam/Adam's fig, herbe de Saint-Christophe/herb Christopher, fève de Saint-Ignace/Ignatius' bean, échelle de Jacob/Jacob's ladder, pain de 
Saint-Jean/St. John's bread, larme-de-Job/Job's tears, herbe à Robert/herb Robert, sceau de Salomon/Solomon's seal, en ichtyologie Jean-doré/ John Dory, etc.

Il arrive cependant que le prénom varie d'une langue à l'autre, comme dans les paires petite angélique/herb Gerard, julienne/Jack-by-the-hedge, véronique/Paul's betony (mais aussi veronica), etc. Plus fréquemment encore, l'une et l'autre langue n'usent pas de prénoms pour désigner les mêmes choses : le français a verge de Jacob (asphodèle jaune), bâton de Saint Jacques (rose trémière), bâton de Saint-Jean (renouée orientale), gros michel (banane plantain), arbre de Moïse (pyracantha) en botanique, etc.; l'anglais, beaucoup plus riche à ce niveau de création, a Aaron's rod (molène commune), Adam's needle (sorte de yucca), creeping jenny (lysimaque nummulaire), Joe-pye-weed (eupatoire pourprée), Noah's night-cap (eschscholzie), Robin-run-in-the-hedge (lierre terrestre) en botanique, Harry-bird (grand puffin), Jack curlew (petit courlis), whiskey Jack (geai commun), Robin snipe (grande maubèche) en ornithologie, Jack-fish (brochet), St Peter's fish (aiglefin) en ichtyologie, spotted Dick (chien dalmatien) en zoologie, etc.

\section{Fonction explétive}

Lorsque le français dit à la tienne, Étienne !, tu parles, Charles !, etc. ou que l'anglais dit have a go, Joe !, believe me, Steve ! etc., le prénom utilisé n'est pas indispensable à la compréhension; il ne sert qu'à renforcer l'interjection tout en lui conférant une tournure plus familière.

On retrouve cette fonction explétive du prénom dans la plupart des autres locutions interjectives : en français, tu vas me le payer, Aglaé !, fonce, Alphonse !, tout juste, Auguste !, recule, Hercule !, tu dis vrai, Jacquet !, relaxe, Max !, cool, Raoul !; en anglais : whoa, Emma !, give me some skin, Flynn !, etc., ainsi que dans certaines locutions adjectivales et adverbiales où le prénom n'est choisi que pour un soutien allitératif ou rimé : en français, à l'hasard, Balthazar, à l'aise, Blaise; en anglais, by guess and by Godfrey, as happy as Larry, as meek as Moses.

On pourrait par analogie considérer comme explétifs les prénoms qui interviennent dans le rhyming slang, partiellement explétifs lorsqu'ils sont porteurs de la rime car dans ce cas la liberté de choix est malgré tout limitée, mais totalement explétifs lorsqu'ils ne font qu'accompagner le mot porteur de rime car alors l'arbitraire est absolu, comme le prouvent les différentes formes qui peuvent exister pour une même notion : Jimmy, Joe et Ned Skinner pour dinner, Harry et Jack Randall pour candle, Dolly et Johnny Cotton pour rotten, Jack et Johnny Horner pour corner, Dicky et Jerry Diddle, Jerry et Jimmy Riddle pour to piddle, etc.

\section{LE CORPUS}

Les entrées du corpus sont définies dans la langue de départ et rendues ensuite soit par un équivalent de la même symbolique soit par une traduction simplement explicative. Puisées tant dans le passé que le présent, elles offrent à côté de créations contemporaines des tournures anciennes, dont certaines sont toujours usitées, d'autres tombées en désuétude. Mais à partir de quel moment peut-on les qualifier d'obsolètes (ob.) puisqu'il en est d'anciennes toujours en usage (jouer les Cassandre, coiffer sainte Catherine, pauvre comme $J o b$, etc.) ? Seules celles se rapportant à des choses ou pratiques disparues peuvent vraiment être considérées comme telles : le bourreau (Jean Guillaume/Jack Ketch), la caisse de bord d'un navire (dominique), la guillotine (bascule à Charlot), être guillotiné (embrasser Charlot), etc. Nous nous bornerons donc à indiquer, chaque fois que faire se peut, la date d'apparition ou la période d'utilisation approximatives de chaque entrée en laissant au lecteur le soin d'apprécier leur éventuelle désuétude en fonction de sa culture 
linguistique propre. De même, nous le laisserons à ses habitudes langagières personnelles pour décider si telle entrée est familière ou populaire, telle autre argotique ou vulgaire, et seules celles que récuse la langue académique seront marquées du signe NC (non conventionnel $\left.{ }^{1}\right)$.

HENRI VAN HOOF

Centre de Terminologie de Bruxelles, Bruxelles, Belgique

\begin{abstract}
Note
1. Dans le sens défini, d'une part, par J. Cellard et A. Rey dans leur Dictionnaire du français non conventionnel et, d'autre part, par E. Partrige dans son Dictionary of Slang and Unconventional English.
\end{abstract}

\title{
SÉLECTION BIBLIOGRAPHIQUE
}

BRUNET, F. et D. MCCAVANA (1996) : Dictionnaire bilingue de l'argot d'aujourd'hui, Paris, Pocket-Langues pour tous.

CELLARD, J. et A. REY (1991) : Dictionnaire du français non conventionnel, Paris, Hachette.

COLIN, J.-P. et J.-P. MÉVEL (1992) : Dictionnaire de l'argot, Paris, Larousse.

DEAK, E. (1962) : Grand dictionnaire d'américanismes, Paris, Éd. du Dauphin.

DE DONY, Y. (1951) : Léxico del Lenguaje figurado, Buenos Aires, Desclée-de Brouwer.

DUNETON, Cl. et S. CLAVAL (1990) : Le Bouquet des expressions imagées, Paris, Le Seuil.

FRANKLYN, J. (1996) : A Dictionary of Rhyming Slang, London, Routledge.

FRIEDRICH, W. (1960) : Moderne deutsche Idiomatik, München, Hueber.

KÜPPER, H. (1955-70) : Wörterbuch der deutschen Umgangssprache (6 Bde), Hamburg, Claasses.

LEITNER, M.J. et J.R. LANEN (1965) : Dictionary of French and American Slang, New York, Crown Publ.

MANSION, J.E. (1980) : Harrap's Standard French-English Dictionary (4 vol.), London, G. Harrap \& C ${ }^{\circ}$.

MARKS, J. (1971) : Harrap's French-English Dictionary of Slang and Colloquialisms, London, G. Harrap \& $\mathrm{C}^{\circ}$. MESSINGER, H. (1959) : Langenscheidts Handwörterbuch Deutsch-English, Berlin, Langenscheidt.

MURET, E., SANDERS, D. und O. SPRINGER (1962) : Langenscheidts Enzyklopädisches Wörterbuch EnglishDeutsch (2 Bde), Berlin, Langenscheidt.

ONIONS, C.T. (1969) : The Shorter Oxford English Dictionary (2 vol.), Oxford, Clarendon Press.

PARTRIDGE, E. (1961) : A Dictionary of Slang and Unconventional English (2 vol.), London, Routledge \& Kegan Paul.

PFOHL, E. und P. BENOÎT (1969) : Brockhaus-Bilderwörterbuch Französisch-Deutsch, Wiesbaden, Brockhaus. PHYTHIAN, B.A. (1986) : A Concise Dictionary of English Slang, London, Hodder \& Stoughton.

SPEARS, R.A. (1991): A Dictionary of Slang and Euphemisms, 2nd ed., New York, Signet. Penguin Books USA.

TAYLOR, R. and W. GOTTSCHALK (1960) : A German-English Dictionary of Idioms, München, Hueber.

\section{ANNEXE 1}

\section{FRANÇAIS}

\section{ACAIRE}

avoir le mal de saint Acaire (1640, ob.) être d'humeur acariâtre; to be badtempered ACHILLE

achille (NC, 1922) couteau; knife

être un Achille $\left(20^{\mathrm{e}}\right)$ être un surhomme; to be a superman talon d'Achille $\left(18^{\mathrm{e}}\right)$ point faible; weak spot, heel of Achilles tendon d'Achille (Anat.)

tendo tricipitis surae; Achilles tendon, tendon of Hector
ADAM

cheville d'Adam (NC, 19)

pénis; the penis, Aaron's rod, Abraham, old Adam, blind Bob, little Davy, dick(y), jack, Jack Robinson, Jenny Donnelly, Jezebel, John (Thomas), johnnie, Lucy, mad Mick, Percy, Peter, Ralph, Roger, Rupert, man Thomas, Timothy, long Tom, Tommy, little Willie

(en) costume d'Adam $\left(19^{\mathrm{e}}\right)$ tout nu; stark naked, Abram, (in) Adam and Eve's togs

figuier d'Adam (Bot.)

bananier du paradis; plantain-tree, Adam's fig 
fourchette d'Adam/du père Adam (1828)

les doigts, la main; the fingers, the hand, Mary Ann

habit du père Adam (1878) nudité; nakedness, Adam and Eve's togs

mouchoir du père Adam (1888) la main; the hand, Mary Ann

ne connaître ni d'Ève ni d'Adam (1752) ne pas connaître du tout; not to know at all, not to know from Adam

nouvel Adam (Rel., 1751)

l'homme en état de grâce; man in a state of grace

peigne d'Adam $\left(19^{\mathrm{e}}\right)$

(cf. mouchoir d'Adam)

pomme d'Adam (Anat., 17 ${ }^{\mathrm{e}}$ ) prominentia laryngea; Adam's apple

se croire sorti de la côte d'Adam

avoir une haute opinion de soi; to have a high opinion of oneself

se mettre dans le costume d'Adam $\left(19^{\mathrm{e}}\right)$

se mettre tout nu; to strip to the buff

vieil Adam (Rel., 1751)

l'homme en état de péché; sinful man, old Adam

AGLAÉ

tu vas me le payer, Aglaé! (1863)

je saurai me venger !; you'll smart for this !

AGNÈS

Agnès $\left(17^{\mathrm{e}}\right.$, ob.)

petite oie blanche; artless young girl

ALEXANDRE

pied d'Alexandre (Bot.)

pyrèthre; feverfew, St. Peter's wort

ALFRED

t'as le bonjour d'Alfred !

formule de refus (1920) ou de congé (1930); a formula of refusal (1920) or leave (1930), Fanny Adams !, my aunt Fanny !, not for Joe !, not for Joseph!

ALICE

ça glisse, Alice ! (1947)

le terrain est glissant (prop. et fig.); it is slippery ground (proper and fig.)

ALONZO

allons-y, Alonzo ! (1950)

mettons-nous au travail; let's set to

partons; let's go

ALPHONSE

Alphonse (NC, 1860)

souteneur; pimp, Alphonse, Charley (Ronce), Joe Ronce, Johnny Ronce, fancy Joseph, Louis, Tommy

fonce, Alphonse ! $\left(20^{\mathrm{e}}\right)$

vas-y!; go ahead

appuie sur le champignon !; step on the gas !

ANASTASIE

Anastasie (1877)

la censure; censorship, Dora

ANATOLE

Anatole

bourreau (NC, 19e); executioner, Jack Ketch président d'assises (NC, 1887); president of an Assize Court

voleur-étrangleur (NC, 1899); thug

ça colle, Anatole ! $\left(20^{\mathrm{e}}\right)$

d'accord !; all right !, O.K. !

c'est parfait !: that's perfect !

ça colle, Anatole ? $\left(20^{\mathrm{e}}\right)$

tout va bien ?, ça marche ?; how's tricks, old dog ?

ANGÉLIQUE

angélique (Bot., $16^{\mathrm{e}}$ )

ombellifère aromatique; an aromatic umbelliferous plant, angelica

angélique sauvage (Bot., $16^{\mathrm{e}}$ )

berce commune; hogweed, wild angelica

petite angélique (Bot., 16 ${ }^{\mathrm{e}}$ )

herbe aux goutteux; goutweed, herb Gerard

ANTOINE

aller de porte en porte comme le pourceau de saint $A n$ toine (1842)

écornifler; to cadge

feu Saint-Antoine (Méd.)

érysipèle; erysipelas, (St.) Anthony's fire

ARLETTE

tu pètes, Arlette ! $\left(20^{\mathrm{e}}\right)$

se dit à qqn qui vient de lâcher un vent; said to s.o. who just broke wind

ARNAUD

avoir son Arnaud (1872)

être de mauvaise humeur; to be grumpy, to be a dismal Jimmy

ARTHUR

Arthur (NC, 1888, ob.)

factionnaire; sentry

appeler Arthur (NC, $20^{\mathrm{e}}$ )

vomir; to vomit, to call for/to cry Charlie, to talk to Earl, to fred, to call/to cry Hughie, to heave up Jonah, to ralph, to call/to cry Ralph, to go to Europe with Ralph and Earl in a Buick, to talk to Ralph on the big white phone, to call/cry Ruth

M(ada)me Arthur (NC, 19)

homosexuel; homosexual, Charley, John-andJoan, Mary Ann, (Miss) Molly, Nancy (boy), Oscar, Tommy Dodd, Willie

se faire appeler Arthur (NC, 1880)

se faire tancer; to get a scolding, to get it where Maggie wore the beads

\section{AUGUSTE}

Auguste

type de clown; sort of clown (1878)

sorte de tulipe ou d'œillet; a kind of tulip or carnation

tout juste, Auguste ! (1900)

c'est bien dit !; you've said it !, Judy!

AUGUSTINE

augustine $\left(19^{\mathrm{e}}\right.$

chaufferette; foot-warmer

pâte à rasoirs; razor paste

étoffe de soie; silk cloth

variété de vigne; a kind of vine

AZOR

Azor 
chien (1771); dog

havresac $(1833$, ob.); knapsack, brown Janet

revolver (1901, ob.); gun, Betsy, John Roscoe,

Maggie

domestique (1926, ob.); man-servant, Jack, John Thomas, Thomas

appeler Azor

huer un acteur (1850); to hiss an actor

siffler pour appeler qqn (1926); to whistle for s.o.

BALTHAZAR

balthazar $\left(19^{\mathrm{e}}\right.$, ob. $)$

repas somptueux; blow-out

à l'hasard, Balthazar ! (1894)

à tout risque; at all hazards

BAPTISTE

baptiste $\left(18^{\mathrm{e}}\right.$, ob. $)$

nigaud; simpleton, silly Billy, Charley, dumbjohn,

Sam, Sammy Soft, simple Simon, Tom Cony, Tom

Fool, Tom Farthing, Tony, silly Willie

tranquille comme Baptiste (1808)

calme et serein; as cool as a cucumber

apathique, indolent; as quiet as a mouse

BASILE

Basile $\left(18^{\mathrm{e}}\right.$, ob.)

calomniateur hypocrite; vile slanderer

BENJAMIN

le benjamin (de la famille) (1808)

l'enfant le plus jeune; the youngest child, the Benjamin

BENOÎT

herbe à Saint-Benô̂t (Bot.) geum urbanum; herb bennet

BENOÎTE

benoîte (Bot.)

(cf. ci-dessus)

BERNARD

Bernard $\left(19^{\mathrm{e}}\right)$

le derrière; the bottom, Fanny, Frances, Nancy, Tom Thumb

aller chez Bernard $\left(19^{\mathrm{e}}\right)$

aller aux toilettes; to go to the toilet, to go to sir Harry, to see Henry, to go to the john, to see John$n y$, to see Lulu

passer par l'arc saint Bernard $(1640, \mathrm{ob}$.) déféquer; to defecate

BERNARDE

bernarde (NC, 1890, ob.) la nuit; the night, Tom Right

BERTHA

grosse Bertha (1918)

canon allemand; German gun, Big Bertha, Long Tom

gros bonnet; bigwig

BERTHE

berthe

collerette; collar, bertha

natte de faux cheveux; flat band of false hair

cruche à lait; milk-can

adieu, Berthe ! (1950)

finies les illusions !, tout est fichu !; it's all over and done with !, all Betty! du temps que Berthe filait (1842)

il y a longtemps; in times bygone, in the days of good Queen Bess, when Adam delved and Eve span

BERTRAND

Bertrand (1825, ob.) coquin; rogue

déchausser Bertrand (1640, ob.) s'enivrer; to get drunk

qui aime Bertrand aime son chien $\left(17^{\mathrm{e}}\right)$

qui m'aime aime aussi les miens; love me, love my dog

BLAISE

à l'aise, Blaise ! doucement !; easy does it !

CARLOS

carlos

cf. Alphonse (NC, 1841)

argent (NC, 1845); money, jack, John Davis, Stephen, Steven

pot de chambre (NC, 1847); chamber-pot, Ellen Terry, jemima, jerry, jordan, Oliver's skull

CAROLINE

Caroline (NC, $20^{\mathrm{e}}$ )

cf. Mme Arthur

travesti; transvestite

CASIMIR

casimir (1790)

tissu sergé croisé; Kerseymere

gilet, waistcoat

CASSANDRE

Cassandre $\left(18^{\mathrm{e}}\right)$ vieillard ridicule; old fogey

jouer les Cassandre $\left(19^{\mathrm{e}}\right)$ prédire des malheurs; to be a bird of ill omen

CATHERINE

coiffer sainte Catherine (1834)

rester vieille fille à vingt-cinq ans; to be unmarried at twenty-five

être Catherine

être pubère; to have attained puberty

prune Sainte-Catherine (Bot.) variété de prune; a variety of plum

CÉLESTIN

voilà un plaisant Célestin ! (1842) en voilà un original !; he's quite a character !

CHARLEMAGNE

Charlemagne (Mil., 1858, ob.)

baïonnette; bayonet, Rosalie

faire Charlemagne $\left(19^{\mathrm{e}}\right)$

se retirer du jeu lorsqu'on gagne; to withdraw from a game when one wins

se croire sorti de la côte de Charlemagne (1640, ob.) (cf. Adam)

CHARLES

Charles (NC)

beau parleur (1640); glib talker

voleur; thief, John Family

Charles le Chauve (NC, 1864)

(cf. cheville d'Adam)

décalotter Charles le Chauve (NC, 1912) 
(se) masturber; to masturbate, Levy and Frank, to fuck Mary Fist

faire sauter la cervelle à Charles le Chauve (NC, 1953) (cf. ci-dessus)

tu est un Charles! (1640)

tu racontes des histoires !;

you're spinning yarns !

tu parles, Charles! (19 $)$

tu l'as dit, bouffi ! now you're talking !, Judy!

CHARLOT

Charlot

cf. Anatole 1 (NC, 1748)

cf. Charles 2 (NC, 1822)

cocard (NC, 1900); black eye

jobard (NC, 1916); dupe, Alec

pauvre type (1970); poor blighter

cf. Charles $1\left(20^{\mathrm{e}}\right)$

grand courlis; great plover (Ornith.)

amuser Charlot (NC, 1977)

(cf. décalotter Charles le Chauve)

bascule à Charlot, boutique de Charlot $\left(\mathrm{NC}, 18^{\mathrm{e}}\right.$, ob.) la guillotine; the guillotine

embrasser Charlot (NC, $18^{\mathrm{e}}$, ob.) être guillotiné; to be guillotined

faire le Charlot $\left(\mathrm{NC}, 20^{\mathrm{e}}\right)$

jouer au plus fin; to try to outwit, to be a smart Alec

soubrettes de Charlot (NC, 1867, ob.)

assistants du bourreau; executioner's aids

CHARLOTTE

charlotte

plat d'entremets (1805); trifle, charlotte

pince à effraction (NC, 1889); crow-bar, jemmy,

jenny, Jim Crow, Jimmy

cf. cheville d'Adam (NC, 1901)

chapeau souple à volants froncés pour femme ou enfant (1905); kind of woman's or children's hat

tu rotes, Charlotte! $\left(20^{\mathrm{e}}\right)$

se dit à qqn qui vient de faire un renvoi; said to s.o. who just belched, nice one Cyril !, way to go Joe ! CHRISTOPHE

herbe de Saint-Christophe (Bot.) actée en épi; baneberry, herb Christopher

CLAIRE

café de sainte Claire café très léger; very light coffee, William

feu Sainte Claire (cf. Elme)

CLAUDE

Claude (1842) (cf. Baptiste)

être bien Claude (1842)

être bien niais; to be quite a ninny, to be a proper Charlie

reine-Claude (Bot.)

variété de prune; greengage

CLÉOPÂTRE

faire Cléopâtre (NC, 1977)

pratiquer la fellation; to fellate

COLAS

Colas cf. Baptiste

corbeau; raven (Ornith.)

sentir la vache à Colas $\left(17^{\mathrm{e}}, \mathrm{ob}\right.$. $)$

être soupçonné d'hérésie; to be suspected of heresy

COLETTE

Jeannot et Colette

couple d'amoureux; loving couple, Edwin and Angelina, Jack and Jill, Darby and Joan

CÔME

heurter à la boutique de saint Côme $(1640$, ob. attraper la vérole; to contract syphilis

patient de saint Côme $(1640, \mathrm{ob}$.) vérolé; syphilitic patient

poivrière de Saint-Côme (NC, 1896) fille vérolée; syphilitic wench

CRÉPIN

c'est tout son saint Crépin (1842, ob.) c'est tout son avoir; it's all his belongings

être dans la prison de saint Crépin $(1640$, ob.) être serré dans ses souliers; to have tight shoes

offre de saint Crépin (1829, ob.) offre qui ne se réalise pas; offer that does not materialize

prendre la voiture de saint Crépin $\left(19^{\mathrm{e}}\right.$, ob. $)$ aller à pied; to foot it

tout le Saint-Crépin (1842, ob.) tout le fourbi; the whole kit and boodle

DAVID

David

crochet de cambrioleur (NC, $15^{\mathrm{e}}$, ob.); picklock Bess, Betty, Kate casquette (NC, 1850-81); cap

étoile de David

étoile des Juifs; Jews' star, Solomon's seal

parent de David qui joue de la harpe $(1640$, ob.) larron; robber

DÉDÉ

dédé (NC, 20

(cf. Bernard)

DENIS

été de la Saint-Denis

derniers beaux jours autour du 9 octobre; last fine days around October 9th, St. Luke's summer

DENISE

elle est revenue, Denise ! (1640, ob.)

exclamation pour marquer le retour d'une femme qui s'était absentée; stock phrase indicating that a woman who had gone away has returned

DIANE

diane

sonnerie de réveil (Mil., $16^{\mathrm{e}}$, ob.); réveille

chant matinal; morning song

papillon diurne; day-butterfly (Ent.)

argent métal; silver (Alch.)

arbre de Diane (Chem.)

dépôt arborescent d'argent (et de mercure); arborescent silver, arbor Dianae

prix de Diane $\left(20^{\mathrm{e}}\right)$

jolie fille; pretty girl, Betty

DOMINIQUE

dominique 


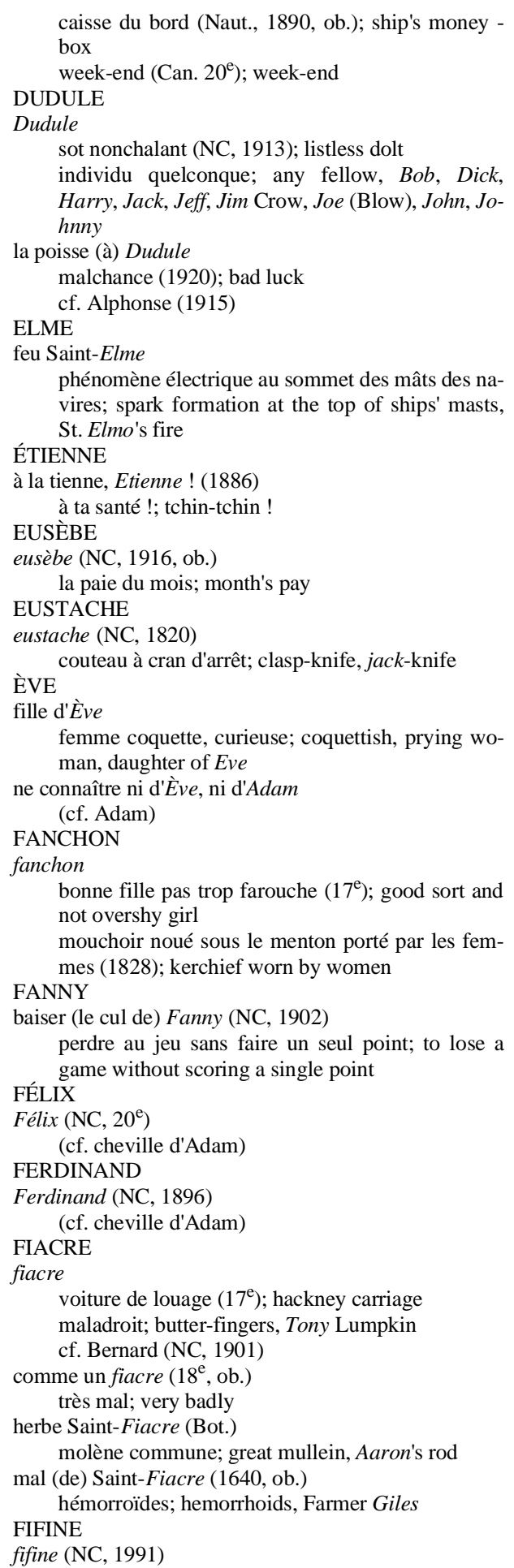

serviette hygiénique; sanitary napkin, fanny pad/ rag, Mickey Mouse mattress, peter-cheater

\section{FRANÇOIS}

le coup du Père François $\left(19^{\mathrm{e}}\right)$

vol à l'étranglement; robbing by throttling coup en traître; treacherous attack

mal Saint-François (1640, ob.)

manque d'argent, pauvreté; shortage of money, poverty

prendre le chariot de saint François

(cf. prendre la voiture de saint Crépin)

FRIDOLIN

Fridolin (NC, 1917)

(soldat) allemand; German (soldier), Freddy, Fritz, Hans, Heinie, Jerry

FRITZ

Fritz (NC, 1914)

(cf. Fridolin)

GALMIER

épaules en bouteille de Saint-Galmier $\left(20^{\mathrm{e}}\right)$ épaules tombantes; sloping shoulders

GASPARD

Gaspard

rat $(\mathrm{NC}, 1833)$; rat

le diable (1842); the devil, old Davy, Saint Nicholas, old Nick

homme malin $(1862$, ob.); cunning fellow, smart Alec

sexe de la femme (NC, 1896); the female genitals, Abraham's bosom, Adam's own, Charley hunt, Eve's custom-house, Fanny, fart-Daniel, Hans Carvel's ring, Jack Nasty-Face, Jack Straw's castle, Lady Jane, Madge, aunt Maria, little Mary, Mary Jane, Molly's hole, mons Meg, mother of St. Patrick, Rufus, tickle-Thomas

cf. cheville d'Adam (NC, 1903)

Dieu dans l'hostie (NC, 1921); God in the host, Jack-in-the-box

avaler le Gaspard (NC, 1953)

communier; to receive Holy communion

GAUTIER

Gautier et Garguille (16 $\mathrm{e}$, ob.) le public; the public at large

ni Gautier ni Garguille $\left(16^{\mathrm{e}}\right.$, ob.) personne; nobody, not a soul

prendre Gautier pour Garguille $\left(16^{\mathrm{e}}\right.$, ob. se tromper de personne; to address the wrong person

se moquer de Gautier et de Garguille $\left(16^{\mathrm{e}}\right.$, ob.) se moquer de tout le monde; not to care for anybody

GENEVIÈVE

c'est sainte Geneviève et saint Marceau (1690, ob.) ce sont des amis inséparables; they are always together, David and Jonathan

pain de sainte Geneviève $(1690$, ob.) guérit de la fièvre; heals fever

GEORGES

laissez faire à Georges, c'est un homme d'âge $\left(17^{\mathrm{e}}\right)$ il faut faire confiance à l'expérience; one must rely on experience, let George do it ! 


\section{GEORGETTE}

(crêpe) georgette (Tex.)

fin tissu de soie; thin silk material, georgette (crepe)

GERTRUDE

Gertrude (NC, Mus., 1906)

contrebasse; double-bass, tuba

GERVAIS

gervais

petit fromage crème; small cream cheese

demi-sel (NC, $20^{\mathrm{e}}$ ); half pimp, half worker

GILLES

Gilles $\left(17^{\mathrm{e}}, \mathrm{ob}.\right)$

(cf. Baptiste)

faire Gilles $(1690, \mathrm{ob}$.

disparaitre, s'enfuir; to abscond, to be off like

Bob's horse

faire banqueroute; to go bankrupt

mal Saint-Gilles (1640, ob.)

cancer; cancer

GINETTE

crêpe Ginette

(cf. Georgette)

GOTON

Goton

fille facile (1809); girl of easy virtue, Kate

fille de campagne, servante (1821); country girl, servant, Joan

GUGUSSE

gugusse $\left(20^{\mathrm{e}}\right)$

1) cf. Auguste

2) crétin; hopeless ass

3) cf. Dudule 2

faire le gugusse

faire le pitre; to play the fool, to make a Judy of oneself, to play the nanny-goat

GUILLAUME

guillaume

pièce de monnaie (ob.); sort of coin

rabot; rabbet-plane

Gros-Guillaume

pain grossier $\left(17^{\mathrm{e}}, \mathrm{ob}.\right)$; coarse bread, brown George

cépage de raisin de table; variety of table grapes

il ressemble au perroquet de Maître Guillaume, il n'en pense pas moins $(1640, \mathrm{ob}$.)

son silence ne signifie pas qu'il n'a pas d'opinion; he has his opinion although he keeps silent

GUILLEMOT

guillemot (Orn.)

oiseau marin; sea-bird, guillemot

du temps du roi Guillemot $(1690$, ob.)

(cf. du temps que Berthe filait)

GUILLOT

être logé chez Guillot le songeur (1640,ob.)

être distrait; to be absent-minded

rêvasser, fantasmer; to indulge in day-dreaming, to be a John-a-dreams

qui croit guiller Guillot, Guillot le guille (1842, ob.)

être le trompeur trompé; harm watch, harm catch
GUSS(E)

guss(e) (NC, 1950)

(cf. Dudule 2)

GUSTAVE

Gustave (NC, 1972)

(cf. Dudule 2)

cravate à Gustave $\left(\mathrm{NC}, 20^{\mathrm{e}}\right)$

GUY

(cf. fifine)

guy (Bot.)

variété de cépage; variety of vine

danse de Saint-Guy (Méd.)

chorée; chorea, St. Anthony's/St. Guy's/St. John's/

St. Vitus' dance

avoir la danse de Saint-Guy $\left(19^{\mathrm{e}}\right)$

s'agiter inefficacement; to play the busybody

HÉLENE

feu Sainte-Hélène

(cf. Elme)

HENRI

chénopode bon-Henri (Bot.) épinard sauvage; blite, good king Henry

\section{HERCULE}

Hercule

homme d'une grande force $\left(16^{\mathrm{e}}\right)$;

a strong man, Hercules, Samson (2) coléoptère géant (Ent.); a gigantic beetle, Hercules beetle

être taillé en Hercule $\left(19^{\mathrm{e}}\right)$

être bien bâti; to be powerfully built

recule, Hercule ! $\left(20^{\mathrm{e}}\right)$ dégage (le terrain) !; make room !

travail d'Hercule $\left(16^{\mathrm{e}}\right)$

entreprise qui exige force et endurance; a task that demands strength and resistance, Herculean task

xanthoxyle massue d'Hercule (Bot.)

xanthoxyle frêne; xanthoxylon, Hercules' club

HUBERT

pain de Saint-Hubert (1690)

guérit de la rage; heals rabies

IGNACE

fève de Saint-Ignace (Bot.)

fève du strychnos ignatier; bean of Strychnos ignatii, Ignatius' bean

INNOCENT

musique de saint Innocent (1640, ob.) cacophonie; cacophony

tulipes de saint Innocent (1640, ob.)

os des morts; bones of buried dead

JACOB

bâton/verge de $J a c o b$

bâton de magicien; conjurer's wand

baudrier d'Orion; Orion's belt (Astr.)

asphodèle jaune; yellow asphodel (Bot.)

échelle de $J a c o b$

échelle céleste; heavenly ladder, Jacob's ladder

échelle de revers (Naut.); leeside ladder, jack-lad-

der

polémonie bleue (Bot.); Greek valerian, Jacob's ladder

JACQUELINE

Jacqueline (NC, 1864) 


\section{JACQUES}

Jacques

sou $(1813$, ob.); small coin

cf. Baptiste (1881)

mollet (1894); calf

cf. Charlotte 2 (NC, 1895)

coffre-fort (NC, 1899); safe, Pete( $r$ )

cf. cheville d'Adam (NC, 19e)

godemiché $\left(\mathrm{NC}, 19^{\mathrm{e}}\right)$; false penis

taximètre (NC, 1935); taximeter

cf. Carlos $3\left(\mathrm{NC}, 20^{\mathrm{e}}\right)$

cf. Jacques Bonhomme

Jacques Bonhomme $\left(14^{\mathrm{e}}\right.$, ob. $)$

sobriquet du paysan français; nickname of the French peasant, Bill Shears, Johnny Whop-Straw, Harry, Jasper, Reuben, Rube

bâton de Saint-Jacques (Bot.) rose trémière; hollycock

battre le Jacques (NC, 1875)

simuler la niaiserie; to feign silliness

coquille Saint-Jacques (Moll.)

pecten; scallop-shell, Jacob's shell

faire le Jacques (1881)

cf. faire le gugusse

se faire valoir; to brag

faire Jacques déloge (1640, ob.)

partir sans payer; to leave without paying

cf. faire Gilles 1

frère Jacques $\left(17^{\mathrm{e}}, \mathrm{ob}\right.$.)

(cf. cheville d'Adam)

herbe de Saint-Jacques (Bot.)

séneçon, jacobée; ragwort, jacobaea

Maître Jacques $\left(17^{\mathrm{e}}\right.$, ob.)

homme à tout faire; bricoleur; handy-man, handy

Andy, Jack of all trades, handy Jack

Pierre, Paul et Jacques

tout le monde; everybody, Tom, Dick and Harry, every Richard

JACQUET

jacquet

domestique; man-servant, Jack, Thomas

écureuil; squirrel

sorte de tric-trac; backgammon

tu dis vrai, Jacquet ! $\left(17^{\mathrm{e}}, \mathrm{ob}\right.$.)

(cf. tu parles, Charles !)

JACQUOT

Jacquot

cf. Jacques 3 (1889)

cf. cheville d'Adam (NC, $19^{\mathrm{e}}$ )

cf. Jacques 4 (NC, 1926)

cf. Jacques 5 (NC, 1926)

cf. Jacques 8 (NC, 1935)

ANOT

perroquet; poll-parrot, Poll, Pretty Poll, Polly

Janot

(cf. Jeannot)

JEAN

Jean $(1640$, ob.)

cf. Baptiste

cocu; cuckold poltron; coward, Charley Howard, Ethel

Jean chouart (NC, 1896)

(cf. cheville d'Adam)

Jean de la vigne $(1867, \mathrm{ob}$.) crucifix; crucifix

Jean des vignes $(1690$, ob. $)$

homme sot et mal avisé; foolish, thoughtless fellow

Jean-doré (Icht.) dorade; dorado, John Dory

Jean-fait-tout (ob.) (cf. Maître Jacques)

Jean farine $(1640, \mathrm{ob}$.) bouffon, comédien; buffoon, comedian, merryAndrew, Jack Pudding

Jean-fesse

bon à rien; good-for-nothing

Jean fichu l'aîné $(1640$, ob.)

(cf. Baptiste)

Jean-foutre (1792)

(cf. Jean-fesse)

Jean Guillaume

(cf. Anatole 1)

Jean-jeudi (NC, 1964)

(cf. cheville d'Adam)

Jean-Lorgne (1842, ob.)

(cf. Baptiste)

Jean-nu-tête (NC, $20^{\mathrm{e}}$ ) (cf. cheville d'Adam)

Jean potage $(1640, \mathrm{ob}$.) (cf. Jean farine)

Jean qui de tout se mêle $(1690$, ob.) importun; bothersome person, Jeff, Melvin

Jean qui ne peut $(1690, \mathrm{ob}$. homme impuissant; impotent male

à la Saint-Jean (cf. en costume d'Adam)

bâton de Saint-Jean (Bot.) renouée orientale; knot-grass

bon Jean $(1640$, ob.)

cf. Jean 2 jambon; ham

c'est comme le bréviaire de Messire Jean (1690, ob.) c'est évident; it's obvious

c'est Gros-Jean qui en remontre à son curé qui ne sait pas veut instruire qui sait; it's like teaching your grandmother to suck eggs

c'est le mariage de Jean des vignes (1690, ob.) concubinage; concubinage

de la Saint-Jean (1829, ob.) sans valeur; worthless

double Jean $(1640, \mathrm{ob}$.

(cf. Jean 2)

employer toutes les herbes de la Saint-Jean $(1690$, ob.) recourir à tous les moyens; to use every means

en saint Jean (1960)

(cf. en costume d'Adam)

être Gros-Jean comme devant $\left(19^{\mathrm{e}}\right)$

n'avoir rien obtenu de ce qu'on espérait; the beggar is back to his hedge again 
faire comme saint Jean, qui donnait le baptême sans l'avoir reçu

(cf. c'est Gros-Jean qui en remontre à son curé)

faire Jean $\left(17^{\mathrm{e}}\right.$, ob.

cocufier; to cuckold

faire Saint-Jean (NC, 1829, ob.)

avertir, donner l'alerte; to warn, to tip off

faire son petit saint Jean

faire le petit saint; to act the little cherub

feu de la Saint-Jean $\left(17^{\mathrm{e}}\right)$

feu de joie (du 24 juin); bonfire (of Midsummernight), St. John's fire

Gros-Jean $\left(18^{\mathrm{e}}\right.$, ob. (cf. Baptiste)

il fait comme le chien de Jean de Nivelle, qui s'enfuit quand on l'appelle $\left(17^{\mathrm{e}}\right)$

il est peu complaisant; he's not very obliging

il ressemble à Messire Jean, qui ne peut lire quand on le regarde $\left(17^{\mathrm{e}}, \mathrm{ob}.\right)$

il est très timide; he's very shy

il est maladroit; he's a butterfingers

mal (de) Saint-Jean (Méd., 1640) épilepsie; epilepsy

pain de saint Jean (Bot.)

fruit du caroubier; fruit of the carob-tree, St. Jo$h n$ 's bread

Petit-Jean $\left(17^{\mathrm{e}}\right.$, ob.)

type du paysan rusé; shrewd peasant type

Saint-Jean (19 ${ }^{\mathrm{e}}$, ob.)

les outils du typographe; the printer's tools

saint Jean bouche d'or (1690, ob.)

bavard (indiscret); telltale

JEANJEAN

Jeanjean

cf. Baptiste (NC)

conscrit niais (NC, 1828, ob.); stupid recruit, Charley

JEANNE

dame-jeanne (1701)

grosse bouteille clissée; carboy, demijohn

marie-jeanne (NC, 1965)

marijuana; marihuana, Mary Ann

JEANNETON

Jeanneton $(1670$, ob.)

servante d'auberge de mœurs peu farouches; serving wench

JEANNETTE

jeannette

petite croix en or (1782); small gold cross

œillet de Pâques (Bot., 1845); pheasant's eye,

poet's narcissus

boîte de botaniste $\left(19^{\mathrm{e}}\right)$; vasculum

bras à repasser (1922); sleeveboard

machine à filer (Tex., ob.); spinning machine, jenny

jeannette blanche (Bot.)

(cf. jeannette 2 )

jeannette jaune (Bot.)

narcisse sauvage; daffodil

JEANNIN

Jeannin (1640, ob.) cf. Baptiste

cf. Jean 2

JEANNOT

Jeannot

(cf. Jeannin)

Jeannot et Colette

(cf. Colette)

JÉROBOAM

jéroboam (1816)

bouteille de la contenance de 4 bouteilles de champagne normales; bottle holding 4 normal champagne bottles, jeroboam

JESSÉ

arbre de Jessé

arbre généalogique du Christ; Christ's family-tree, tree of Jesse

JÉSUS

jésus

format de papier (1633); paper size

jeune homosexuel (NC, 1835); young homosexual, $\operatorname{Nan}(c y)$ boy

jeune enfant innocent (1840); young innocent child

cf. cheville d'Adam (NC, 1919)

couteau de poche (NC, 1919); pocket-knife, jackknife

Jésus, Marie, Joseph !

exclamation de surprise, de répulsion, etc.; an exclamation of surprise, repulsion, etc., Jesus !, (holy) Moses !

beau comme un Jésus de cire $\left(19^{\mathrm{e}}\right)$

d'une beauté délicate; pretty and delicate (features) en Jésus

(cf. en costume d'Adam)

p'tit Jésus (NC, 20 ${ }^{\mathrm{e}}$ )

JOB

(cf. cheville d'Adam)

ami de $\operatorname{Job}\left(17^{\mathrm{e}}\right)$

consolateur pessimiste; pessimistic friend, Job's comforter

femme à $J o b\left(17^{\mathrm{e}}\right)$

femme acariâtre; shrewish woman, Jezebel, Job's wife

larme-de-Job (Bot.)

larmille; species of grass (Coix lacryma), Job's tear

patience de $J o b$ (1690)

extrême patience; great endurance, patience of $J o b$

pauvre comme $J o b\left(15^{\mathrm{e}}\right)$

très pauvre; utterly poor, as poor as $J o b$ ('s cat/turkey)

JOJO

affeux jojo (NC, 1930)

vilain garnement; little monster

individu peu fréquentable; disreputable fellow, Jack Nasty-Face

faire son jojo (NC, 1867)

se montrer puritain; to act the prude, to play Jose$p h$

faire la bête, l'enfant; to act foolishly, childishly 


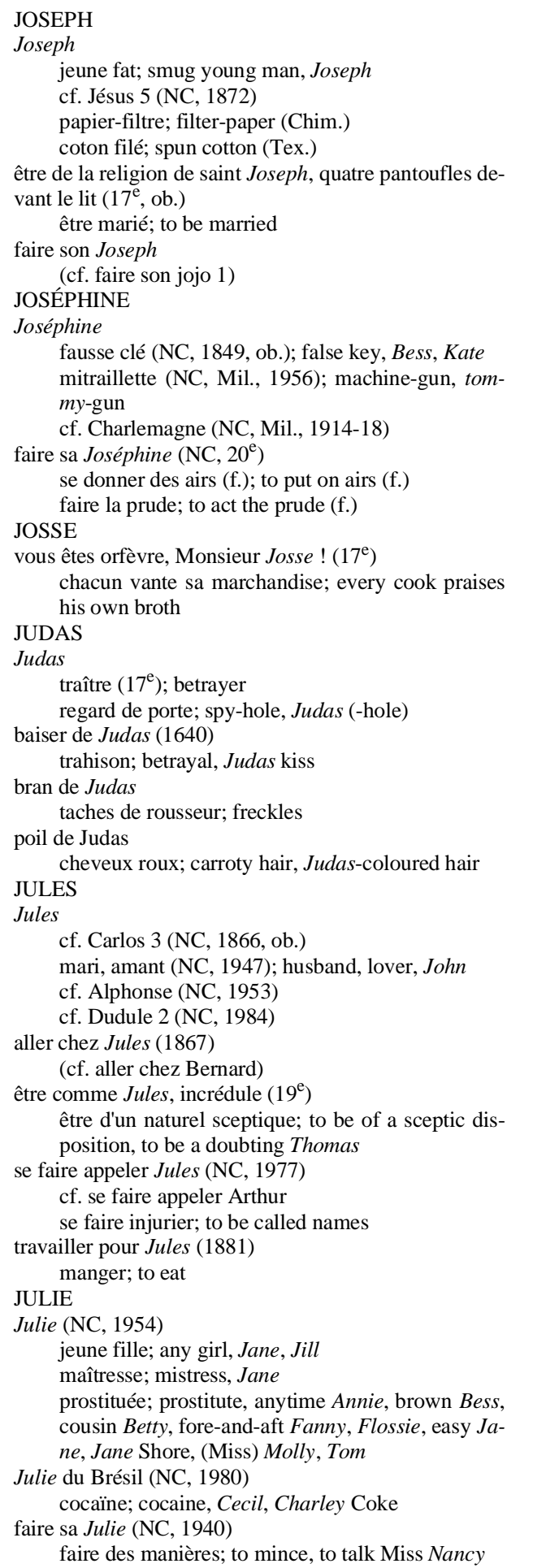

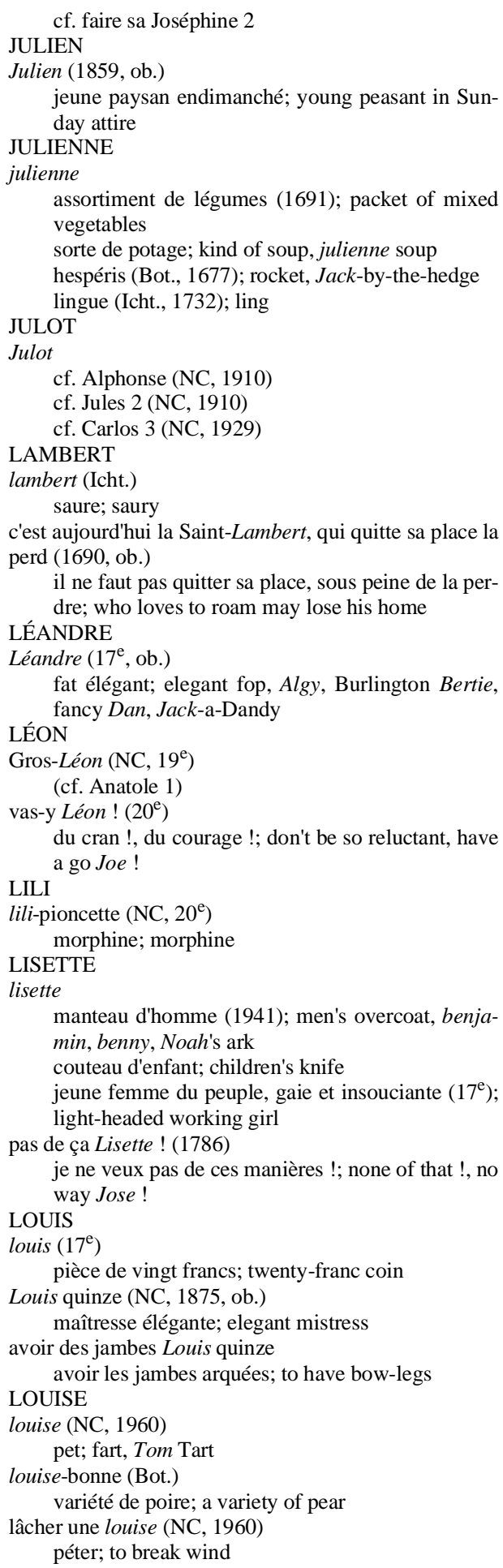




\section{LOUISETTE}

louisette (Bot.)

gesse tubéreuse; vetch

\section{LUBIN}

lubin

domestique rural $(1821, \mathrm{ob}$.$) ; country servant$ bar commun (Icht.); sea-perch

Frère Lubin $(1640, \mathrm{ob}$.

moine niais; simple-minded monk

LUC

luc $\left(\mathrm{NC}, 18^{\mathrm{e}}\right)$

anus; anus

cf. Bernard

cela n'est pas selon saint Luc

ce n'est pas conforme aux règles; that's not cricket

léger comme l'oiseau saint $\operatorname{Luc}(1640, \mathrm{ob}$.)

lourd comme un bœuf; stupid, loutish

Messire $L u c\left(\mathrm{NC}, 18^{\mathrm{e}}\right)$

(cf. Bernard)

LUCE

à la Sainte-Luce, les jours croissent d'un saut de puce (1842)

proverbe relatif à l'allongement des jours après le solstice d'hiver; a saying about the lengthening of days after the winter solstice

LUCETTE

crêpe Lucette

(cf. Georgette)

LUCRÈCE

faire la Lucrèce $\left(17^{\mathrm{e}}\right)$

(cf. faire sa Joséphine 2)

\section{MACAIRE}

c'est un scrupule de saint Macaire $(1842$, ob.)

c'est un scrupule absurde, une bigoterie ridicule; it

is an absurd scruple, ridiculous bigotry

MADELEINE

madeleine

variété de poire (Bot., 1701); a sort of pear, Mag-

dalen pear

biscuit de Savoie (1769); sponge-cake

sorte de cépage (Bot., $18^{\mathrm{e}}$ ); a variety of vine

pécheresse repentie $(1867, \mathrm{ob}$.); reformed prostitute, Magdalen

faire suer la Madeleine (NC, 1878, ob.)

gagner laborieusement au jeu en trichant; to win laboriously by cheating (games)

pleurer comme une Madeleine (1833)

pleurer à chaudes larmes; to cry one's eyes out

MAHOMET

Mahomet

bourse suspendue sur la poitrine (NC, 1855, ob.); purse hanging on the chest

soleil (d'Afrique) (NC, Mil., 1866); the (African) sun, Betsy

mal de Mahomet $\left(17^{\mathrm{e}}\right.$, ob.

la goutte; gout

si la montagne ne vient pas à Mahomet, Mahomet ira à la montagne

faire naître une occasion qui ne se présenterait pas d'elle-même; to raise an opportunity that would not occur by itself, if the mountain will not go to

Mahomet, let Mahomet go to the mountain

MARCEAU

c'est sainte Geneviève et saint Marceau (cf. Geneviève)

MARCEL

Marcel

ami de cœur (NC, 1986); sweetheart, John

maillot de corps ajouré (NC, 1988); net singlet

MARCELINE

marceline

sorte de taffetas (Tex.); sort of taffeta

oxyde naturel de manganèse (Chim.); natural manganese oxide

MARGOT

Margot $\left(17^{\mathrm{e}}, \mathrm{ob}.\right)$

pie (Orn.); magpie

femme bavarde; chatterbox

cf. Goton 1

MARGUERITE

marguerite

bellis vivace (14') ; daisy, herb Margaret

cordage (Naut., $17^{\mathrm{e}}$ ); deck-tackle

outil de corroyeur (19 $)$; graining board

préservatif (NC, 1927); condom, jimmy hat, (rub-

ber) johnnie, johnnie bag, willie

cheveu blanc (NC, 1977); white hair

marguerite de la Saint-Michel

aster annuel; wild aster, Michaelmas daisy

marguerites de cimetière

(cf. marguerite 5)

MARIANNE

Marianne (1860)

la République française; the French Republic

avoir la Marianne dans l'œil (1867)

être ivre; to be drunk, to be blind as Chloe, to be as drunk as David's sow

MARIE

Marie-Chantal $\left(20^{\mathrm{e}}\right)$

jeune femme maniérée; affected young lady, Tottie fie

Marie-couche-toi-là (NC, 1750)

(cf. Goton 1)

marie-galante (Bot.)

quinquina corymbifère; corymbiferous cinchona

Marie-gercée (NC, 1984)

(cf. Marie-Chantal)

Marie-graillon

femme laide et malpropre; plain and slovenly woman

marie-jeanne

(cf. Jeanne)

marie-louise (NC, 1977)

(cf. Louise)

Marie-pisse-trois-gouttes (NC, 1977)

fille très jeune; very young girl

marie-salope

bateau-drague (Naut., 1777); dredge-boat

femme malpropre (NC, 1836); slut, dolly

cf. Julie 3 (NC, 1877)

cuisine roulante (Mil., 20e); field kitchen 
vodka et jus de tomates (NC, 1977); vodka and tomato juice

chardon Marie (Bot.)

chardon argenté; milk-thistle

enfant de Marie (19)

individu naï; guileless fellow

fleur de Marie (1836) virginité; maidenhead

marin de la Vierge Marie (19 ${ }^{\mathrm{e}}$, ob.) marin d'eau douce; land-lubber

violette de Marie (Bot.) sorte de campanule; Campanula medium

MARIETTE

mariette (Bot.)

(cf. violette de Marie)

MARION

marion (Bot.)

(cf. Louisette)

être ensemble comme Robin et Marion (1842)

être en parfaite intelligence; to be on good terms with

MARTIN

Martin

surnom de l'âne $\left(15^{\mathrm{e}}\right)$; nickname given to a donkey, dicky, jackass, Neddy

genre d'étourneau (Orn., 16 ${ }^{\mathrm{e}}$ ); kind of starling

martin-bâton (1640, ob.) gourdin; cudgel, billy

martin-pêcheur (Orn., 1573)

Alcedo ipsida; kingfisher

martin rose(lin) (Orn., 16 ${ }^{\mathrm{e}}$ )

Pastor roseus; rose starling

martin-sec/-sire/-sucré (Bot.)

variété de poire; a variety of pear, Martin dry

à chaque porc vient la Saint-Martin

à chacun son tour; every dog has his day

du même Saint-Martin

du pareil au même; six of one, half a dozen of the other

estaffier de saint Martin (1690, ob.)

(cf. Gaspard 2)

été de la Saint-Martin

derniers beaux jours; Indian summer, St. Martin's summer

faire la Saint-Martin (1690, ob.)

faire ripaille; to feast

il n'y a point de Martin qu'il n'y ait de l'âne (1640, ob.)

les imbéciles sont légion; silly asses are everywhere

il y a plus d'un âne (à la foire) qui s'appelle Martin (1690)

il y a plus d'une personne qui porte le même nom the same name is borne by many, there are more Jacks than one at the fair

mal saint Martin (1690, ob.) ivresse; drunkenness

ours Martin

ourson en peluche; children's toy, Teddy bear

plus d'un âne à la foire s'appelle Martin (1842)

il faut être prudent dans ce qu'on affirme; one must be cautious as to what one asserts pour un point Martin perdit son âne $\left(17^{\mathrm{e}}\right)$

pour peu de chose on perd son fait; for want of a nail the shoe was lost

prendre Martin pour Renard $\left(17^{\mathrm{e}}\right.$, ob.) (cf. prendre Gautier pour Garguille)

prêtre Martin qui chante et qui répond (1842, ob.) (cf. Jean qui de tout se mêle)

tirer saint Martin par l'épaule (1640,ob.)

ôter le manteau; to take off one's coat

MATHIEU

Mathieu

campeur sans chic (NC, 1953); unstylish camper alpiniste novice (NC, 1961); inexperienced mountaineer

fesse-mathieu $\left(17^{\mathrm{e}}\right)$

usurier; shylock, Ike, Ikey

avare; niggard

fesser Mathieu (1640, ob.)

prêter à usure; to lend money at usurious interests

vieux comme Mathieu-salé (1867, ob.)

(cf. Mathusalem)

MATHURIN

Mathurin (NC, 1847)

marin; sailor; Jack (Nasty-Face), Jack Tar, tarry John

mathurins

dés à jouer (NC, 1821); dice

dents (jaunies) (NC, 1878); (yellow) teeth

mathurins plats (NC, 1836)

dominos; dominoes

avoir des tranchées de saint Mathurin (1640, ob.) avoir des accès de folie; to have fits of madness

colique/mal saint Mathurin (1640, ob.) folie; madness

envoyer qqn à saint Mathurin $(1640, \mathrm{ob}$. faire passer pour fou; to give s.o. out for mad

faire trimer les mathurins $(\mathrm{NC}, 1878)$ (cf. travailler pour Jules)

MATHUSALEM

mathusalem $\left(19^{\mathrm{e}}\right)$ bouteille d'une contenance équivalente à huit bouteilles de champagne normales; bottle of champagne containing eight normal bottles

vieux comme Mathusalem $\left(19^{\mathrm{e}}\right)$

très vieux; very old, as old as Adam, as old as Methuselah

du temps de Mathusalem (cf. du temps que Berthe filait)

MAX

$\operatorname{relax}(\mathrm{e}), \operatorname{Max} !\left(20^{\mathrm{e}}\right)$

du calme !, détends-toi !; calm down !, relax !

MÉDARD

faire la mine de saint Médard $(1690, \mathrm{ob}$. grimacer;to make a face

rire de saint Médard (1690, ob.) rire forcé; wry smile

s'il pleut le jour de la Saint-Médard, il pleut quarante jours plus tard (1842)

dicton relatif à l'influence de ce saint sur le temps; a saying about the saint's influence upon the weather 
MICHEL

Michel Bonhomme

le bourgeois allemand; the middle-class German, Fritz

faire la rue Michel (1890, ob.)

faire le compte; to tally

gros michel (Bot.)

banane plantain; plantain (banana)

marguerite de la Saint-Michel

(cf. marguerite)

Saint-Michel (Bot.)

variété de poire; a variety of pear

traquenard saint Michel $(1690$, ob.)

(cf. Gaspard 2)

vendre des coquilles à ceux qui viennent de Saint- $M i$ chel

faire qqch d'inutile; to carry coals to Newcastle, to help uncle Anthony to kill dead mice

\section{MICKEY}

Mickey $\left(\mathrm{NC}, 20^{\mathrm{e}}\right)$

cf. Jean-fesse

boisson frelatée; adulterated drink

balançoire à Mickey (NC, 1965)

$$
\text { (cf. fifine) }
$$

MIMI

$\operatorname{mimi}(\mathrm{NC}, 1931)$

(cf. Gaspard 4)

MIMILE

Mimile

(cf. Baptiste)

MINERVE

minerve

cerveau, esprit $\left(17^{\mathrm{e}}\right.$, ob. $)$;

brain(s)

femme belle et intelligente $(1812$, ob.); beautiful, intelligent woman

collier orthopédique (Méd., 1840); orthopedic collar

avoir de la minerve $\left(17^{\mathrm{e}}, \mathrm{ob}.\right)$

être intelligent; to have brains

porter des chouettes à Minerve

(cf. vendre des coquilles à ceux qui viennent de Saint-Michel)

rimer malgré Minerve

rimailler; to write uninspired verse, to write

"invita Minerva"

MOHAMED

Mohamed

cf. Mahomet (NC, 1955)

Arabe (NC, 1988); Arab, Abdul

MOISE

moïse (1889)

berceau-corbeille; basket cot, Moses basket

arbre de Moïse (Bot.)

pyracantha; Egyptian thorn, pyracanth

parent de Moïse (1640, ob.)

(cf. Jean 2)

MONIQUE

monique $\left(\mathrm{NC}, 20^{\mathrm{e}}\right)$

(cf. Gaspard 4)
NABUCHODONOSOR

nabuchodonosor $\left(19^{\mathrm{e}}\right)$

bouteille d'une contenance de 20 bouteilles de champagne normales; bottle holding 20 normal champagne bottles, nabuchodonosor

NAPOLÉON

napoléon $\left(19^{\mathrm{e}}\right)$

pièce de vingt francs-or; twenty-franc gold piece

NARCISSE

Narcisse

homme amoureux de lui-même $\left(17^{\mathrm{e}}\right)$; male in love with his own beauty, Narcissus

genette (Bot., 14 ${ }^{\mathrm{e}}$ ); daffodil, narcissus narcisse sauvage (Bot.)

(cf. jeannette jaune)

NÉNETTE

nénette

jeune fille attrayante (NC, 1954); attractive girl cf. Minerve 1 (NC, 1930)

NESTOR

Nestor $\left(16^{\mathrm{e}}, \mathrm{ob}.\right)$

vieillard sage et éloquent; wise, eloquent old man

NICAISE

(un vrai) Nicaise

(cf. Baptiste)

NICODÈME

Nicodème (1842)

(cf. Baptiste)

NICOLAS

Nicolas $\left(19^{\mathrm{e}}\right)$

(cf. Baptiste)

Nicolas j't'embrouille ! (1867)

exclamation de défi; an exclamation of challenge

feu Saint-Nicolas

$$
\text { (cf. Elme) }
$$

pain de Saint-Nicolas $(1690$, ob.)

pain censé guérir de diverses infirmités; bread claimed to heal various physical disabilities

NINON

coiffure à la Ninon

NOE

cheveux coupés sur la nuque; bobbed hair

arche de Noé $\left(17^{\mathrm{e}}\right)$

maison abritant plusieurs ménages; house where several families live

lieu à grand public; crowded place

lieu de refuge; place of refuge

il est des enfants de Noé, de la race des " ja fait " (1690,

ob.)

il est dépensier; he's a spendthrift

OTHON

alléluia d'Othon (1842, ob.)

vantardise; piece of bounce

PANCRACE

docteur Pancrace $\left(17^{\mathrm{e}}\right.$, ob.)

faux savant; false scientist

ergoteur; champion arguer

PASCAL

Pascal (NC, 20 ${ }^{\mathrm{e}}$ )

(cf. cheville d'Adam) 


\section{PATRICE}

Patrice et Mario (NC, 20 ${ }^{\mathrm{e}}$ )

équipe de deux policiers qui patrouillent ensemble; team of two policemen making the rounds

PAUL

Paul et Virginie (18 ${ }^{\mathrm{e}}$ (cf. Jeannot et Colette)

découvrir saint Pierre pour couvrir saint Paul $\left(17^{\mathrm{e}}\right)$ dérober à l'un pour donner à l'autre; to steal from one to give to another, to rob Peter to pay Paul

frayer avec Pierre et Paul

être liant avec tout le monde; to make friends with everybody, to be hail-fellow-well-met with Tom, Dick and Harry

Pierre et Paul; Pierre, Paul et Jacques (cf. Jacques)

prendre saint Pierre pour saint Paul (cf. prendre Gautier pour Garguille)

saint-paul (Bot.) variété de cépage noir; a variety of black vine saintpaulia (Bot.) genre de gesnéracées; a genus of gesneracaea

\section{PAULINE}

faire pleurer Pauline (NC, $\left.20^{\mathrm{e}}\right)$

uriner; to urinate, to Dicky

Diddle, to have a Jimmy Riddle,

to Jerry Riddle, to joe, to Mike

Bliss, to point Percy at the porcelain, to train Terrence on the terracotta

PAULO

Paulo $\left(20^{\mathrm{e}}\right)$

(cf. Dudule 2)

vas-y Paulo ! $\left(20^{\mathrm{e}}\right)$

(cf. vas-y Léon!)

PÉNÉLOPE $\left(16^{\mathrm{e}}\right)$

Pénélope $\left(16^{\mathrm{e}}\right)$

femme vertueuse; virtuous woman, Penelope espèce de gallinacé d'Amérique centrale; a genus of gallinaceous birds in Central America, penelope

c'est la toile de Pénélope ! (1842)

c'est un travail sans cesse à recommencer; it is a never-ending task

\section{PERRETTE}

boîte à Perrette $\left(17^{\mathrm{e}}, \mathrm{ob}\right.$. $)$ tirelire; money-box

fonds secrets; secret funds

c'est Perrette et le pot au lait ! $\left(17^{\mathrm{e}}\right)$ (cf. adieu, Berthe!)

PÉTRUS

Pétrus (NC, 1926) (cf. Bernard)

PHILÉMON

philémon (Orn.) espèce de passereau de Malaisie; a genus of passerines in Malaysia

Philémon et Baucis (cf. Jeannot et Colette)

PHILIBERT

Philibert carambouilleur (NC, 1836, ob.); swindler cf. cheville d'Adam $\left(\mathrm{NC}, 20^{\mathrm{e}}\right)$

PHILIDOR

Philidor (NC, $20^{\mathrm{e}}$ )

(cf. cheville d'Adam)

PHILIPPE

philippe

écu $(1821$, ob.); crown (three or five-franc piece)

vol au rendez-moi (NC, 1882, ob.); ringing the change

PIERRE

Pierre et Paul; Pierre, Paul et Jacques (cf. Jacques)

découvrir saint Pierre pour couvrir saint Paul (cf. Paul)

denier de saint Pierre

contribution volontaire des catholiques au trésor papal; voluntary contributions of the Roman $\mathrm{Ca}$ tholics to the papal treasury, Peter('s) penny

hardi comme un saint Pierre $(1842$, ob.)

menteur effronté; shameless liar

prendre saint Pierre pour saint Paul (cf. Paul)

(cf. frayer avec Pierre et Paul)

se lier avec Pierre et Paul

(cf. frayer avec Pierre et $\mathrm{Paul}$ )

PIERROT

pierrot

paysan $(1668$, ob.); bumpkin, Bill Shears, hillbilly, Harry, Jasper, Johnny Whop-Straw, Reuben, Rube

moineau (Orn., 1694); sparrow

personnage de la comédie italienne $\left(17^{\mathrm{e}}\right)$; character of the Commedia dell'Arte

cf. Baptiste $(1834$, ob.)

verre de vin blanc $(1867, \mathrm{ob}$.$) ; glass of white wine$ individu bizarre (1892); queer fellow

recrue (Mil, 1865-88); recruit, Joe Blow, John(nie Raw)

vieux soldat (1890-1932); old soldier

asphyxier le pierrot $\left(19^{\mathrm{e}}, \mathrm{ob}\right.$. $)$

boire un verre de vin blanc; to drink a glass of white wine

un drôle de pierrot

(cf. pierrot 6)

être gai comme un pierrot $\left(17^{\mathrm{e}}\right)$

être de joyeuse humeur; to be in a merry mood, to be as happy as Larry

faire le Pierrot

(cf. faire le Jacques)

PONCE

(r)envoyer qqn de Ponce à Pilate (1640)

ballotter qqn de l'un à l'autre;

to drive s.o. from pillar to post

POPAUL, POPOL

Popaul, Popol (NC, 1975)

(cf. cheville d'Adam)

boîte à Popaul (NC, $\left.20^{\mathrm{e}}\right)$ (cf. Gaspard 4)

étrangler Popaul (NC, 1982)

(cf. décalotter Charles le Chauve)

imperméable à Popaul $\left(\mathrm{NC}, 20^{\mathrm{e}}\right)$

(cf. Marguerite 4) 


\section{PROSPER}

Prosper

cf. Eusèbe (NC, 1889, ob.)

cf. Alphonse (NC, 1946)

ordinateur central de la police (NC, 1975); central police computer

RAMONA

Ramona (NC, 1808, ob.)

petit ramoneur; chimney-boy

chanter Ramona à qqn (NC, 1930, ob.)

réprimander qqn; to take s.o. to task

RAOUL

raoul $\left(\mathrm{NC}, 20^{\mathrm{e}}\right)$

démodé, hors du coup; old-fashioned

cool, Raoul! $\left(20^{\mathrm{e}}\right)$

(cf. relax, Max !)

RAYMOND

raymond $\left(\mathrm{NC}, 20^{\mathrm{e}}\right)$

(cf. Raoul)

RÉBECCA

rébecca $\left(\mathrm{NC}, 18^{\mathrm{e}}\right)$

protestation violente; fierce protest

faire le $(1781) / \mathrm{du}(1807) / \mathrm{sa}\left(20^{\mathrm{e}}\right)$ rébecca

protester, regimber; to kick (over the traces), to raise Cain

\section{RENARD}

Renard

cf. Gaspard $3\left(17^{\mathrm{e}}\right)$

espion, traître (1829); sneak, betrayer

briseur de grève (1909);strike-breaker

compère le renard, maître renard $\left(13^{\mathrm{e}}\right)$

l'animal rusé des fables; the sly fox in tales, Reynard (the Fox)

prendre Martin pour Renard

(cf. prendre Gautier pour Garguille)

RENÉ

Gros-René $\left(17^{\mathrm{e}}\right.$, ob. $)$

serviteur de confiance; confidential servant

se chauffer à la cheminée du roi René $(1842$, ob.) se chauffer au soleil; to bask in the sun

\section{RICHARD}

Richard sans peur $\left(17^{\mathrm{e}}, \mathrm{ob}\right.$. $)$

homme courageux, hardi; courageous, fearless man

la science du Bonhomme Richard $\left(18^{\mathrm{e}}\right)$

conseils de bon sens; advice prompted by common sense, Poor Richard's sayings

\section{ROBERT}

robert

cf. Charlot 3 (NC)

sein de femme (NC, 1928); female breast, Char-

leys, Lewis and Witties, lulus, murphies, Norma Snockers

roberts de chez Michelin (NC, $20^{\mathrm{e}}$ )

faux seins; false breasts

herbe à Robert (Bot.)

géranium, bec-de-grue; crane's bill, geranium, $\mathrm{Ro}$ bert's bill, herb Robert

métal du prince Robert (Chem., $17^{\mathrm{e}}$ )

cuivre raffiné; refined copper, Prince Rupert's metal sauce Robert

sauce relevée à base d'oignons et de moutarde; seasoned sauce made with onions and mustard

ROBIN

robin

cf. Pierrot 1 (ob.)

homme de peu (ob.); low-born fellow

cf. Jean farine

taureau reproducteur; bull for service

chanson de Robin (1640, ob.)

discours ennuyeux; boring speech

faire comme Robin fit à la danse $\left(17^{\mathrm{e}}, \mathrm{ob}\right.$. $)$

faire de son mieux; to do one's best

un plaisant Robin (1690, ob.)

(cf. Célestin)

toujours souvient Robin de ses flûtes

se souvenir toujours de l'important (1606, ob.); always to remember the main thing

on revient facilement à d'anciennes habitudes (1842, ob.); old habits die hard

vivre ensemble comme Robin et Marion

(cf. Marion)

$\mathrm{ROCH}$

c'est saint Roch et son chien (1690)

(cf. c'est sainte Geneviève et saint Marceau)

être comme saint Roch en chapeau (1842, ob.)

être pourvu de tout; to be blessed with everything

ROGER

Roger Bontemps (1690, ob.)

joyeux drille; happy-go-lucky fellow, good-time Charley

ROLAND

mourir de la mort de Roland $\left(17^{\mathrm{e}}\right.$, ob.) mourir de soif; to die of thirst

faire le Roland (1640, ob.)

faire le vaillant; to come the brave menacer; to threaten

ROMÉO

Roméo

amoureux; lover

boisson faite de rhum et d'eau (NC, $20^{\mathrm{e}}$ ); rum and water drink

ROSALIE

Rosalie (NC, Mil., 20 $0^{\mathrm{e}}$

(cf. Charlemagne)

poitrine de Tante Rosalie dessert à la gélatine; gelatin dessert, shivering $\mathrm{Liz}$

SALMANAZAR

salmanazar $\left(19^{\mathrm{e}}\right)$

bouteille d'une contenance de 12 bouteilles de champagne normales; bottle holding 12 normal champagne bottles, salmanazar

SALOMON

Salomon $\left(16^{\mathrm{e}}\right)$

homme sage; wise man, Solomon

il ressemble le sage Salomon, il vient des champs chier à la maison $\left(17^{\mathrm{e}}, \mathrm{ob}\right.$. $)$

il fait le discret; he acts modestly

jugement de Salomon

jugement équitable; fair judgment, justice of Solomon 
sagesse de Salomon

grande sagesse; great wisdom, wisdom of Solomon

sceau de Salomon (Bot.)

muguet anguleux; Polygonatum vulgare, Solomon's seal

SAM

(1') oncle $\operatorname{Sam}\left(19^{\mathrm{e}}\right)$

les États-Unis, le peuple américain; the United States, the American people, Brother Jonathan, Uncle Sam

SAMSON

épée de Samson $\left(17^{\mathrm{e}}\right.$, ob.) machoîres; the jaws

s'escrimer des armes de Samson (1690, ob.) (cf. travailler pour Jules)

SIMON

aller chez Simon $(\mathrm{NC}, 1867)$ (cf. aller chez Bernard)

SIMONE

simone (NC, 1902) police des jeux; police in charge of gambling

en voiture, Simone ! $\left(20^{\mathrm{e}}\right)$ (cf. allons-y, Alonzo !)

faire la simone $(1835, \mathrm{ob}$.) extorquer des aumônes; to extort alms SOPHIE

faire sa Sophie (NC, 1878) (cf. faire sa Julie 1)

voir Sophie (NC, 1867) avoir ses règles; to have one's menses

SYLVESTRE

on le verra à la Saint-Sylvestre

il n'arrivera jamais à l'heure; he wouldn't be in time for his own funeral

TEDDY

Teddy $\left(20^{\mathrm{e}}\right)$

manteau en fourrure synthétique; synthetic fur coat

blouson brodé dans le dos; embroidered jumperblouse

THIBAUT

mal Thibaut mitaine $\left(17^{\mathrm{e}}\right.$, ob. la bêtise; stupidity

THOMAS

Thomas (NC, 1830) (cf. Carlos 3)

aller voir la mère/veuve Thomas $\left(\mathrm{NC}, 19^{\mathrm{e}}\right)$ (cf. aller chez Bernard) avoir avalé Thomas (NC, 1881, ob.)

avoir une haleine fétide; to have a foul breath

être comme (saint) Thomas

(cf. être comme Jules, incrédule)

faire ronfler Thomas (1867) déféquer; to defecate, to george, to tom-tit

la mère (1847)/veuve (1867) Thomas (NC, ob.) chaise percée; night commode, Sir Harry

un saint-Thomas

un incrédule; a sceptic, a doubting Thomas

TOM

tom-pouce

personne de petite taille $\left(19^{\mathrm{e}}\right)$; undersized person, Jack Sprat, Tom Thumb parapluie très court (1924); short umbrella, Tom Thumb umbrella

TOMMY

Tommy (1914-18) soldat britannique; British soldier, Tommy

VÉRONIQUE

véronique

thé d'Europe (Bot., 16e); speedwell, Paul's betony, veronica

suaire $\left(13^{\mathrm{e}}\right)$; shroud, veronica

passe en tauromachie; a pass in bull-fighting

VICTORIA

victoria

variété de pigeon domestique (1879); a species of domestic pigeon, Victoria

voiture découverte à quatre roues (1870); fourwheeled carriage with collapsible hood, victoria

Victoria regia (Bot., 1854)

maïs d'eau; water-maize, Victoria regia

VIOLETTE

violette

genre de violacées $\left(\right.$ Bot., $12^{\mathrm{e}}$ ); a plant of the genus

Viola, violet

type de suivante $\left(18^{\mathrm{e}}, \mathrm{ob}\right.$.); waiting-maid

violette de Marie

(cf. mariette)

faire sa violette

affecter une modestie exagérée; to affect an excessive modesty, to play the shrinking violet

YVAN

Yvan (NC, 20 $\left.{ }^{\mathrm{e}}\right)$

sobriquet du Russe; nickname for a Russian, Ivan

ZACHARIE

mal Saint-Zacharie (1640, ob.)

le silence; silence

\section{ANGLAIS}

AARON

Aaron's beard (Bot.)

Hypericum calycinum; mille-pertuis velu Aaron's rod (Bot., 1834)

great mullein; molène commune, herbe Saint-Fiacre

golden rod; verge d'or

cf. Abraham (19th)
$\mathrm{ABE}$

Abe's cabe (US, NC, 1935)

five-dollar bill; billet de cinq dollars

brown Abe (US, NC, 1935)

penny; pièce d'un penny

ABEL

Cain and Abel (RS, 1857)

table; table 
ABDUL

Abdul (20th)

ABIE

Arab; Arabe, Mohammed

Abie (US, NC, 1920)

any male Jew; Juif de sexe masculin tailor; tailleur

ABIGAIL

Abigail (17th)

lady's maid; soubrette

ABRAHAM

Abraham (19-20th, ob.)

the penis; pénis, cheville d'Adam, Charles le Chauve, Charlotte, Félix, Ferdinand, frère Jacques, Gaspard, Jacquot, Jean chouart, Jean-jeudi, Jean-nu-tête, Pascal, Philibert, Philidor, Popaul, p'tit Jésus

Abraham's balm (Bot.) chaste tree; petit-poivre

Abraham's bosom (1870) the female genitals; sexe de la femme, Gaspard, mimi, monique, boîte à Popaul

Abraham's willing (RS, 1859) shilling; pièce d'un shilling

to feel as in Abraham's bosom (ob.) to live in affluence; vivre dans l'abondance

to sham Abraham (16th, ob.) to feign sickness; faire le malade

there's no leaping from Dahlila's lap into Abraham's bosom (ob.)

one cannot serve two masters; on ne peut servir deux maîtres

ABRAM

abram (17-18th)

naked; nu, en costume d'Adam, en Jésus

ACHILLES

Achilles tendon (Anat.)

tendo tricipitis surae; tendon d'Achille

heel of Achilles

ADA

weak spot, weak side; point faible, talon d'Achille

Frigging Ada ! (NC, 20th)

an exclamation or oath; exclamation ou juron, En-

ADAM fer et Belzébuth! Jésus, Marie, Joseph!

Adam's ale (17-20th)

water; eau, Château-la-Pompe

Adam's apple (Anat.)

laryngeal prominence; cartilage thyroïde, pomme d'Adam

Adam and Eve

type of orchid; sorte d'orchidée (Bot.)

to believe (RS, 1914); croire

to leave (RS, 19-20th); partir

to Adam and Eve it

to coit; coïter (NC, 19th)

to practise nudism (US, NC); faire du nudisme

Adam and Eve on a raft (US, NC, 20th) eggs on toast; œufs sur toast

Adam and Eve's togs $(1909$, ob.) nakedness; nudité, costume d'Adam
Adam's arsenal (NC, 1870)

penis and testicles; pénis et testicules

Adam's fig (Bot.) plantain-tree; bananier du paradis, figuier d'Adam

Adam's flannel (Bot.)

(cf. Aaron's rod 1)

Adam's needle (Bot.) genus of yucca; espèce de yucca shepherd's needle; scandix

Adam's own (NC, 19th)

(cf. Abraham's bosom)

as old as Adam

very old; vieux comme le monde, vieux comme Mathusalem

at the time when Adam delved and Eve span

in times bygone; il y a longtemps,du temps que Berthe filait, du temps de Mathusalem

to cast off the old Adam

to renounce one's former life; dépouiller le vieil homme

not to know better than dead and gone Adam

not to know the first word of; n'en pas savoir le premier mot

not to know from Adam (18-20th)

not to know at all; ne pas connaître du tout, ne connaître ni d'Ève ni d'Adam

(the) old Adam

sinful man; l'homme pécheur, le vieil Adam

cf. Abraham (19-20th)

since Adam was a boy

since the beginning of things; depuis que le monde est monde

we are all Adam's children, but silk makes the differen-

ce

fine feathers make fine birds; c'est l'habit qui fait le moine

what Eve did with Adam (NC, 19th)

copulation; coït

ALEC

Alec (NC, 1925)

dupe; dupe, jobard, Charlot

smart Alec (1873)

sly fellow; finaud, Gaspard, Renard

know-all; monsieur je-sais-tout

ALGY

$\operatorname{Algy}$ (19th, ob.)

swell, dude; jeune gommeux, Léandre

ALICE

knock three times and ask for Alice (20th)

a catch-phrase to cut short long-drawn-out directions (as to location); se dit pour couper court à de longues explications

sweet Alice (US, NC, 20th)

glass of milk; verre de lait

ALPHONSE

Alphonse (RS, 19-20th)

pimp; proxénète, Alphonse, Carlos, poisse à $\mathrm{Du}$ dule, Jules, Julot, Prosper

AMY-JOHN

Amy-John (US, NC, 20th)

lesbian; lesbienne 


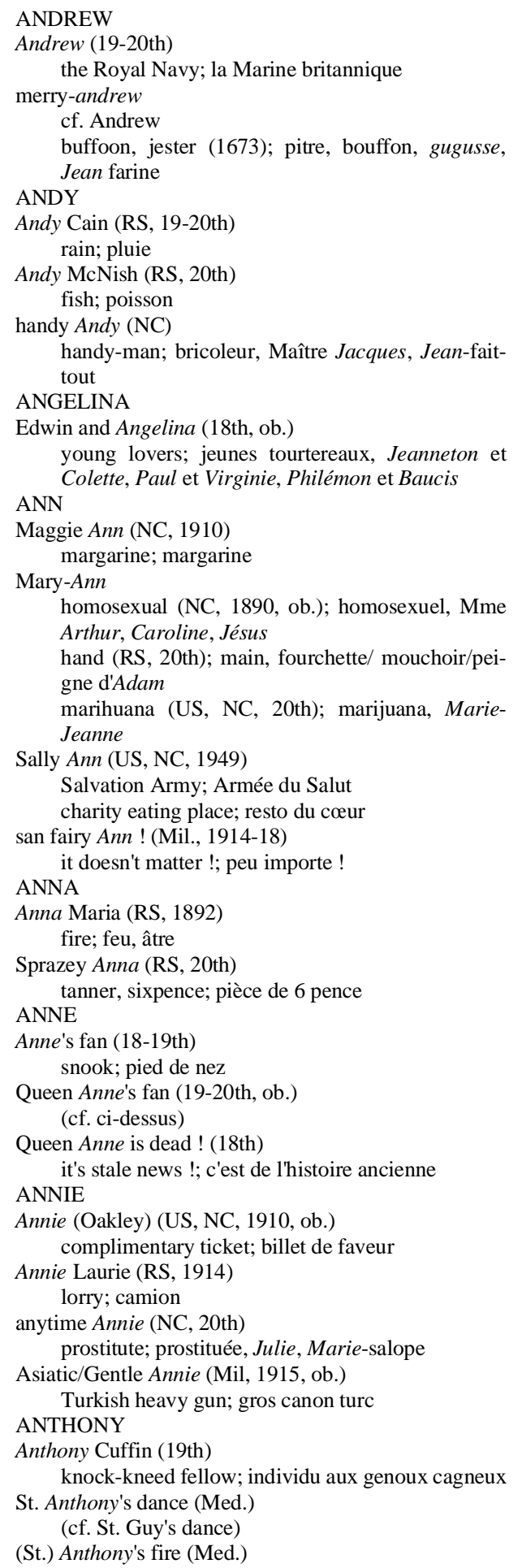

erysipelas; érysipèle, feu Saint-Antoine to cuff/knock Anthony (18-19th)

to walk knock-kneed; marcher les genoux en dedans

to dine with St. Anthony (1749, ob.)

to go without eating; se passer de manger

to help Uncle Anthony to kill dead mice (20th)

to carry coals to Newcastle; faire qqch d'inutile, perdre son temps, vendre des coquilles à qui vient de Saint-Michel, porter des chouettes à Minerve

ANTONIO

Antonio

Portuguese soldier (Mil., NC, 1914, ob.); soldat portugais

Italian (NC); Italien

ARCHIBALD

Archibald, Archie (Mil., NC, 1915)

anti-aircraft gun; canon anti-aérien

Archibald, certainly not! (NC, 1913-20)

definitely not !; certainement pas !, jamais de la vie!

BARNABY

Barnaby Rudge (RS, 20th)

judge; juge

Barnaby thistle (Bot.)

BEN

Centauraea solstitialis; centaurée du solstice

Ben Flake (RS, 1855-1910)

steak; bifteck

Big Ben

the big bell of Parliament (1856); la grosse cloche du Parlement (Londres), Big Ben ten (pounds) (RS, 20th); dix livres

brother Ben, co-pilot Ben (US, NC, 20th)

benzedrine; benzédrine

BENEDICT

Benedict (19-20th)

newly-wed fellow; nouveau marié

BENJAMIN

Benjamin

the youngest (of a family or group); le plus jeune (d'une famille, d'un groupe), le benjamin

benzoin (Bot.); benjoin

overcoat (NC, 1815); pardessus, lisette

husband (NC, 1870); mari

benjamin-bush/-tree (Bot.)

benzoin laurel; styrax benjoin

Benjamin's mess (16th)

the larger part; la plus grosse part

BENNY

Benny

cf. Benjamin 3 (ob.)

hat (ob.); chapeau

amphetamine (NC, 20th); amphétamine

BERTHA

bertha (1856)

collar; collerette (de corsage), berthe

Big Bertha (1), big Bertha (2)

heavy German gun (1917); gros canon allemand, grosse Bertha

fat woman; grosse dondon 
BERTIE

Burlington Bertie (NC, 1909-60)

BESS

$$
\text { (cf. Algy) }
$$

Bess (NC, 17-19th)

picklock; rossignol, David, Joséphine

black Bess (NC, 19th)

(cf. Abraham's bosom)

brown Bess

cf. anytime Annie (17th, ob.)

regulation rifle (Mil., 18-19th); fusil réglementaire yes (RS, 1859, ob.); oui

to hug brown Bess (1780-1850)

to serve in the Army; embrasser la carrière militaire

in the days of good Queen Bess

(cf. at the time when Adam delved)

BETSY

Betsy (US, NC, 1935)

gun; revolver, Azor; rifle, fusil

sun; soleil, Mahomet, Mohamed

Black Betsy (US, NC, 20th)

baseball bat; batte de baseball

BETTY

Betty (NC)

cf. Bess (17-19th)

man assuming a woman's domestic duties (19-

20th); tâte-au-pot

good-looking girl (US, 20th); jolie fille, prix de Diane

cf. Mary-Ann 1 (US, 20th)

Betty Lea/Lee (RS, 20th)

tea; thé

all Betty! (19th)

it's all up !; tout est perdu !, adieu, Berthe !, c'est

Perrette et le pot au lait !

all my eye and Betty Martin! (1785)

nonsense !, rubbish !; des blagues !, foutaises !

cousin Betty

cf. anytime Annie (18-19th, ob.)

BILL

half-witted female (19-20th, ob.); demi-douce

Bill Harris (Mil., 1914-16)

bilharziosis; bilharziose

Bill Shears (US, NC, 1920)

farmer, rustic; paysan, rustaud, Jacques Bonhomme, pierrot

the old Bill (20th)

the police; la police

Uncle Bill (20th)

idem.

BILLY

billy

tin-can, (tea-)kettle (1850); gamelle, bouilloire (à thé)

club, cudgel (1874); gourdin, matraque, martin-

bâton

bowler hat (1887); chapeau melon

slubbing machine (Tex., 18th); boudineuse

he-goat (20th); bouc

goatee (20th); barbichette locomotive; locomotive

Billy Button (RS, 1857-1940)

mutton; mouton

billycock (1862)

(cf.Billy 3)

billy-doo (18-20th)

love-letter; billet doux

billy-goat

cf. Billy 5 (1861)

cf. Billy 6 (1882)

Billy Noodle (NC, 1920)

a fellow who thinks all girls are in love with him; séducteur imaginaire

Billy Turniptop (1890, ob.) agricultural labourer; ouvrier agricole

Billy Webb (Bot.)

a papilionaceous plant; sorte de papilionacée (Swertia panamensis)

hill(-)billy (US, 1956)

(cf. Bill Shears)

like billy-o(h), like old Billy (1894)

like hell; diablement

puffing Billy

cf. Billy 7 (19-20th)

person short of breath (20th); personne à l'haleine courte

silly Billy (NC, 19-20th)

simpleton, ninny; benêt, nigaud, Baptiste, Claude,

Colas, Jacques, Janot, Jean, Jeannin, Jeannot, Nicaise, Nicodème, Nicolas, Pierrot

whistling Billy (19-20th)

(cf. Billy 7)

bob (NC)

any fellow (1700, ob.); individu quelconque, $\mathrm{Du}$ dule, guss(e), Jules

cf. Abraham's willing (1810)

Bob, Harry and Dick (RS, 1868, ob.) sick; malade

Bob's your uncle ! (1890, ob.) everything's all right !; tout va bien !

all is $b o b !(\mathrm{NC}, 17-19$ th $)$

(cf. above)

off like $B o b$ 's horse with nobody to pay the reckoning (1830, ob.)

to decamp with all the money, furniture and personal effects; filer avec l'argent et les meubles, faire Gilles, faire Jacques-déloge

old blind Bob (NC, 20th)

BOBBY

(cf. Abraham)

bobby (1851)

policeman; policier

bobby's job (Mil., 1915-18)

CAIN

easy job; sinécure

Cain and Abel (cf. Abel)

to raise Cain (1840)

to make a hell of a noise; faire un boucan de tous les diables 
to kick; rouspéter, faire du rébecca

to wear the mark of Cain

to be branded with infamy; être marqué du sceau de l'infamie

CATHERINE

Catherine wheel

cart-wheel; roue (Gym.)

rose-window; rosace (Arch.)

to do a Catherine-wheel, to turn Catherine wheels

(Gym., 1850)

to turn cart-wheels; faire la roue

CECIL

Cecil (US, NC, 20th)

cocaine; cocaïne, Julie du Brésil

CHARLES

Charles, charles (US, NC, 20th) (cf. Cecil)

Charles's Wain (Astr.) the Plough; la Grande Ourse

\section{CHARLEY}

Charley

watchman $(1805$, ob. $)$; veilleur

cf. Billy 6 (1830)

fox (1857); renard

joint or muscle stiffness (US, NC, 1910); raideur

articulaire ou musculaire

cf. Alphonse (NC, 1920)

stupid soldier (US, 1945); soldat stupide, Jeanjean

cf. Many-Ann 1 (1945)

cf. anytime Annie (1846)

Charleys (NC)

female breasts (1840); seins, roberts

testicles (20th); testicules

Charley Brady (RS, 19-20th, ob.)

hat, cady; chapeau

Charley Coke (US, NC, 1953, ob.) cf. Cecil

cocaine addict; cocaïnomane

Charley Dilke (RS, 1880-1940) milk; lait

Charley Freer (RS, 1903-30) beer; bière

Charley Frisky (RS, 1860-1910) whisky; whisky

Charley horse (US) (cf. Charley 4)

Charley Howard (RS, 20th) coward; poltron, Jean

Charley Hunt (RS, 1880) (cf. Abraham's bosom)

Charley Lancaster (RS, 1857) handkerchief; mouchoir

Charley Mason (RS, 1880) basin; bassin

Charley Pope (RS, 1914) soap; savon

Charley Prescott (RS, 1857) waistcoat; gilet

Charley Randy (RS, 1860-1910) brandy; eau-de-vie

Charley Ronce (RS, 19-20th) (cf. Alphonse)

Charley Skinner (RS, 1860-1910)

dinner; dîner

Charley Wiggins (RS, 19-20th)

lodgings; logement

cheap Charley

travelling hawker; camelot

good-time Charley (US, NC)

happy-go-lucky fellow; joyeux drille, Roger Bontemps

to introduce Charley (NC, 20th)

to coit; coïter

to poke Charley at s.o. (NC, 1935)

to make fun of s.o.; se payer la tête de qqn

CHARLIE (cf. also Charley)

Charlie (US, NC) (cf. Cecil)

Charlie Brown! (US, NC, 20th)

(cf. Frigging Ada !)

Mr. Charlie (US, NC, 19-20th)

any white man in Negro slang; tout homme blanc dans l'argot des Noirs

to call for/cry Charlie (NC, 20th)

to vomit; vomir, appeler Arthur

proper Charlie (cf. Silly Billy)

to look a proper Charlie to look stupid; avoir l'air fin, être bien Claude

CHARLOTTE

charlotte (Cul., 1855)

trifle; plat d'entremets, charlotte

good time Charlotte

(cf. anytime Annie)

CHLOE

blind (1780-1860)/drunk (1815-20th) as Chloe extremely drunk; complètement ivre, avoir $M a$ rianne dans l'œil

CHRISTOPHER

Christopher Columbus ! (US, 19-20th) (cf. Frigging Ada !)

CLARENCE

Clarence (20th, ob.)

a government employee shirking military service; fonctionnaire embusqué pour échapper au service militaire

COLLEEN

Colleen Bawn (RS, 1862) erect penis; érection

CORINE

Corine (US, NC, 20th)

(cf. Cecil)

CYRIL

nice one, Cyril ! (20th)

said to s.o. who just belched; se dit à qqn qui vient de faire un renvoi, tu rotes Charlotte!

DAHLILA

there's no leaping from Dahlila's lap into Abraham's bosom

(cf. Abraham)

DAISY

daisy 
Bellis vivace (16th); marguerite

a first-rate person or thing (1876); personne ou chose remarquable

daisy-beaters (NC, 19th) feet; pieds

daisy-cutter (18-19th, ob.)

horse; cheval

Daisy Dormer (RS, 20th)

warmer; plus chaud

daisy recruits (RS, 1855-70), daisy roots (RS, 1874) boots; souliers

Gert and Daisy (RS, 19-20th)

lazy; paresseux

Michaelmas daisy (Bot.) wild aster; aster annuel, marguerite de la SaintMichel

to pick a daisy (NC, 1860)

to defecate in the open air or to retire to urinate (esp. f.); déféquer en plein air ou se retirer pour

DAN uriner (spéc. f.)

Dan Tucker (RS, 1859-1950)

butter; beurre

fancy Dan (US, NC)

cf. Algy (1950)

skilful boxer; boxeur habile

DANIEL

Daniel

upright judge; juge intègre

man with an unerring judgement; homme au jugement infaillible

fart-Daniel (NC, 19th)

(cf. Abraham's bosom)

good Godfrey Daniel! (US, 19-20th) (cf. Frigging Ada!)

to take one's Daniel (1860-1900)

to depart or decamp; partir ou décamper

DAVID

David, david (19-20th)

affidavit; déclaration sous serment

David and Jonathan

inseparable friends; amis inséparables, c'est sainte Geneviève et saint Marceau, c'est saint Roch et son chien

David Jones (1751) the spirit of the sea; le génie de la mer

as drunk as David's sow (17th, ob.) (cf. blind as Chloe)

DAVY

Davy, davy (1764) (cf. David)

Davy Crockett (RS, 20th) pocket; poche

Davy's dust (Naut., 1830) gunpowder; poudre à canon

Davy's/Davy Jones' locker (Naut., 18th) the bottom of the sea; le fond de la mer

Davy Jones (cf. David Jones)

Davy Large (RS, 19-20th) barge; chaland to go to Davy Jones' locker (18th) to get drowned at sea; périr en mer little Davy (NC, 19th)

(cf. Abraham)

old Davy (1870)

the devil; le diable, Gaspard

to take one's davy on it (18th)

to give one's word for it; en donner sa parole

DICK

dick (NC)

dictionary (US 1860, GB 1870); dictionnaire

cf. Abraham (1860)

cf. David (1861)

cf. Bob 1 (16-20th)

detective (1930); détective

cf. Bobby (1939)

to dick around (US, NC, 20th)

to idle, loaf about; fainéanter, perdre son temps

Dick's hatband (19-20th, ob.)

makeshift; expédient, pis-aller

dick-sucker (US, NC, 20th)

(cf. Mary Ann 1)

Dick, Tom and Harry (16-20th)

everybody; tout le monde, Pierre et Paul, Pierre, Paul et Jacques

any Tom, Dick or Harry

anybody; le premier venu, n'importe qui

Bob, Harry and Dick

(cf. Bob)

clever Dick (NC, 1923)

(cf. smart Alec 1)

clipped Dick (US, NC, 19-20th)

Jew; Juif

every Tom, Dick and Harry

(cf. Dick, Tom and Harry)

every swinging dick (US, 20th)

(cf. Dick, Tom and Harry)

in the days/reign of Queen Dick (17th, ob.) never; jamais

King Dick (RS, 19-20th)

brick; brique

one law for Dick and another for Harry one law for the rich and another for the poor; deux poids et deux mesures

spotted Dick

currant pudding (1849); pudding aux raisins

dalmatian dog; dalmatien

Tom, Dick and Harry (1920)

(cf. Dick, Tom and Harry)

Uncle Dick (RS)

cf. Bob, Harry and Dick (19-20th)

cf. Abraham (20th)

DICKY

dicky

male ass (1793); âne, martin

little bird (1851); petit oiseau

cf. Abraham

Dicky Diddle (RS, 20th)

to urinate; uriner, faire pleurer Pauline

Dicky Dirt (RS, 19-20th)

shirt; chemise 


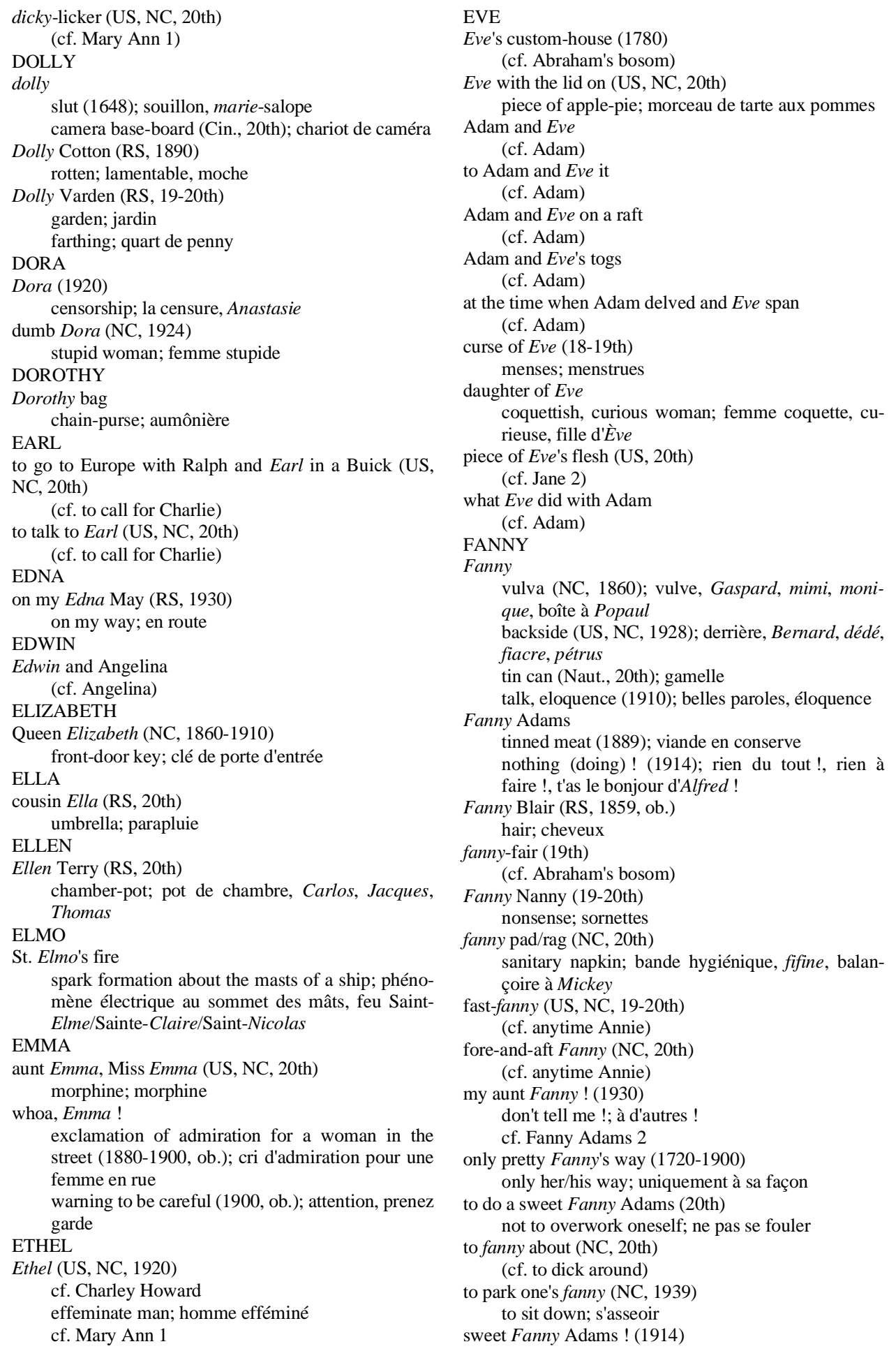


nothing (doing) !; rien du tout !, rien à faire !, t'as le bonjour d'Alfred !

to wiggle one's fanny (NC, 20th)

FLO

to hurry up; se dépêcher

aunt Flo (US, 20th)

(cf. curse of Eve)

visit from Flo (US, 20th)

(cf. curse of Eve)

FLOEY

drunk as Floey (19-20th)

(cf. blind as Chloe)

FLOSSIE

Flossie (NC, 20th)

(cf. anytime Annie)

FRANCES

Frances (US, NC, 20th)

(cf. Fanny 2)

FRANK

Levy and Frank (RS, 1880)

masturbation, whank; onanisme, décalotter Charles-le-Chauve, faire sauter la cervelle à Charlesle-Chauve, étrangler Popaul

\section{FRED}

Fred (US, NC, 20th)

social outcast; paumé

to fred (US, NC, 20th)

(cf. to call for Charlie)

Uncle Fred (RS, 20th)

bread; pain

FREDDIE

blind Freddie would see it ! (1930)

it's staring you in the face !; cela crève les yeux !

ready for Freddie (US, NC, 20th)

ready for the unexpected or the unusual; s'attendre à tout, être prêt à tout

FREDDY

Freddy (1914-18, ob.)

German soldier; soldat allemand, Fridolin, Fritz any German; tout Allemand

FRITZ

Fritz (1914-18)

(cf. Freddy)

to be on the fritz (NC, 1928)

out of order; hors service, en panne

GABRIEL

Gabriel (US, NC, 20th) trumpet player; trompettiste

GEOFFREY

on St. Geoffrey's day (1786-1850)

(cf. in the days of Queen Dick)

GEORGE

George, george

penny or guinea $(1870, \mathrm{ob}$.$) ; penny ou guinée$ aviator (1915); aviateur

automatic pilot (1928); pilote automatique

householder; chef de famille

cf. Daisy 2 (1950)

to george (US, NC, 20th)

to defecate; déféquer; faire ronfler Thomas

(by) George ! (18-20th) (cf. Charlie Brown!)

big George (US, NC)

a quarter; pièce de 25 cents

brown George

coarse brown bread (17-19th); pain gris grossier,

Gros-Guillaume

brown wig (19th); perruque brune

jug (1860); cruchon

let George do it (1910)

leave it to the expert; laissez faire les connaisseurs, laissez faire à Georges

on the George Robey (RS, 1910)

on the road, on the toby; sur la route

yellow George (1785, ob.)

(cf. George 1)

GEORGETTE

georgette (crepe) (Tex., 1920)

thin silk material; fin tissu de soie, crêpe georgette/ginettellucette

GEORGY

Georgy-Porgy (1870)

any plump boy; garçonnet un peu boulot

GERARD

herb Gerard (Bot.)

goutweed; herbe aux goutteux, petite angélique

GERT

Gert and Daisy (RS, 19-20th)

GERTIE (cf. Daisy)

Gertie Gitana (RS, 19-1950)

banana; banane

giddy Gertie (US, 20th)

stupid girl; fille stupide

GERTRUDE

Gertrude (Cin., 1930)

steel crane with a camera at its head; grue de prise de vues

GILBERT

over the Gilbert (NC, 20th)

gone wrong; (cela a) mal tourné

GILES

to dine with St. Giles and the Earl of Murray (18-20th, ob.)

(cf. to dine with St. Anthony)

Farmer Giles (RS, 20th)

piles; hémorrhoïdes, mal de Saint-Fiacre

hopping Giles (1785, ob.)

cripple, limping fellow; infirme, boiteux

GODFREY

by guess and by Godfrey (1909)

at random; au hasard, à l'aventure

(good) Godfrey!, Godfrey mighty !, Gum Godfrey!

(US, 1880-20th)

(cf. Frigging Ada!)

good Godfrey Daniel! (US, 19-20th)

(cf. Frigging Ada !)

GORDON

Gordon and Gotch (RS, 20th)

watch; montre

GUSSIE

Dear Gussie ! (US, 20th) 


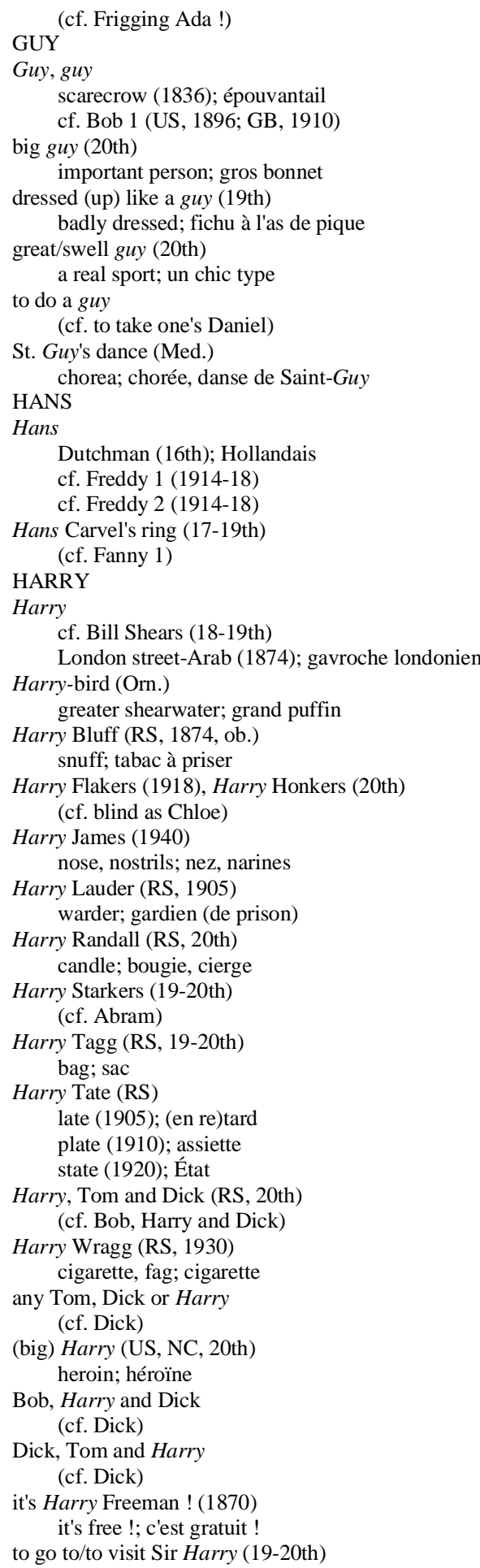

to go to the toilet; aller aux toilettes, aller chez Bernard/Jules/Simon

old Harry $(1740$, ob.)

(cf. old Davy)

one law for Dick and another for Harry (cf. Dick)

to play old Harry (with s.o.) (1837)

to give s.o. a rough time; en faire voir de grises à qqn

cf. to raise Cain 2

Sir Harry

night-commode, toilet (19-20th); chaise percée, la mère Thomas, WC, Jules

constipation (1923, ob.); constipation

Tom, Dick and Harry (cf. Dick)

what Harry gave Doll (18-19th)

(cf. what Eve did with Adam)

HARVEY

Harvey Nichol (RS, 1932)

pickle; situation difficile

HAZEL

aunt Hazel (US, NC, 20th)

(cf. big Harry)

HECTOR

Hector (1720)

bully, swashbuckler; bravache, matamore

Hector's pecking (RS, 20th) necking; bécotage, pelotage

since Hector was a pup

(cf. since Adam was a boy)

tendon of Hector (Med.)

(cf. Achilles tendon)

to wear Hector's cloak (17-18th)

to be rightly rewarded for one's treachery; être puni pour sa traîtrise

HEINIE

Heinie (NC, 1914-18) (cf. Freddy)

HELEN

go to Helen B. Happy ! (US, 1910) go to hell (and be happy) !; allez au diable !

HENRY

Henry (US, NC, 1920)

Ford car; automobile Ford

Henry, henry (US, NC, 20th)

(cf. big Harry)

Henry Nash (RS, 20th)

cash (money); argent comptant

good King Henry (Bot.)

blite; épinard sauvage, chénopode bon-Henri

Hooray Henry (20th)

fast-living toff; jeune noceur de la haute

to look for Henry (NC, 1920)

to look for a dupe; chercher un pigeon (au jeu)

to see Henry (US, 20th)

(cf. to go to sir Harry)

HERBERT

Herbert (20th)

(cf. Bob 1) 


\section{HERCULES}

Hercules

strong man (1567); homme d'une grande force,

Hercule

cf. Hercules beetle

Hercules beetle (Ent., 1816)

a gigantic lamellicorn beetle; coléoptère géant, hercule (des Antilles)

Hercules' club (Bot., 1882)

a species of xanthoxylon; xanthoxyle frêne, xanthoxyle massue d'Hercule

HERMAN

Herman (US, NC, 1950) (cf. Bob 1)

Herman Fink (RS, 20th)

ink; encre

HUGHIE

to call for/cry Hughie (NC, 20th)

(cf. to call for Charlie)

HUMPHREY

to dine with Duke Humphrey (16-20th, ob.) (cf. to dine with St. Anthony)

IGNATIUS

Ignatius' bean, bean of St. Ignatius (Bot., 1751) bean of Strychnos ignatii; fève du strychnos ignaIKE tier, fève de Saint-Ignace

Ike (US, NC, ob.)

cf. Abie 1 (1864)

cf. silly Billy (1895)

usurer (20th); usurier, fesse-mathieu

alibi Ike (US, 20th)

one who always offers excuses for his actions; qqn qui trouve toujours des excuses à ses agissements

Mike and Ike (US, NC, 20th)

salt-box and pepper-box; salière et poivrier

IKEY

Ikey

cf. Ike 1

cf. Ike 3

conceited fellow; prétentieux

ISABELLA

Isabella (RS, 1855)

(cf. cousin Ella)

IVAN

Ivan (Mil., 1914-20)

Russian (soldier); (soldat) russe, Yvan

JACK

Jack, jack

sailor (1659); marin, mathurin

knave (of cards) (1674); valet (aux cartes)

cf. Bob 1 (1746, ob.)

man-servant (1836); valet, Jacquet

cf. Sir Harry (18-19th, ob.)

cf. Abraham (19-20th, ob.)

money (1937); argent, carlos

cf. big Harry

cf. bobby

informer; indicateur

Jack Adams (17-19th)

(cf. silly Billy)
Jack-a-dandy (1630-1920)

(cf. Algy)

Jack afloat (18th)

(cf. Jack 1)

Jack's alive (RS, 20th)

five (pounds); cinq livres

Jack and Jill (RS)

bill (1890); note, facture

hill (20th); colline

pill (20th); pilule, cachet

cf. Edwin and Angelina

jackass (1727)

(cf. dicky 1)

Jack Blunt (1898-1910) blunt fellow; individu bourru

Jack Brag (16-20th, ob.) braggart; vantard

Jack-by-the-hedge (Bot.) hedge garlic; alliaire, julienne

Jack curlew (Orn) whimbrel; petit courlis

Jack Dandy (RS, 1857) (cf. Charley Randy)

jackdaw (Orn., 1543) a bird of the crow type; corneille des clochers

Jackdaw in peacock's feathers one who takes pride in what he has borrowed from others; geai paré des plumes du paon

Jack-fish (Icht.) pike; brochet

Jack Frost (1825, ob.) frosty weather; Bonhomme Hiver

Jack-go-to-bed-at-noon (Bot.) goat's beard; salsifis des prés

Jack Horner (RS, 20th) corner; coin

Jack in office (17th) imperious petty official; petit bureaucrate autoritaire

Jack-in-the-basket (Naut.) danger-buoy; vigie

Jack-in-the-box consecrated host (1546, ob.); hostie consacrée, Gaspard

sharper, cheat (1570-1830); escroc

toy (17th); boîte à surprise

mere puppet (1702); fantoche

child in woman's womb; enfant porté dans le ventre

syphilis, pox (RS, 1870); syphilis

cf. Abraham (19-20th, ob.)

Jack-in-the-bush (Bot.) (cf. Jack-by-the-hedge)

Jack-in-the-cellar (1750-1900) (cf. Jack-in-the-box 5)

Jack-in-the-pulpits (US, Bot.) Arisaema triphyllum; liliacée sauvage

Jack Johnson (Mil., 1914-18, ob.) heavy German gun or shell; gros canon ou obus allemand

Jack Jones (RS, 20th) 
alone; seul

Jack Ketch (1705-1880)

executioner; bourreau, Charlot, Jean Guillaume

jack-knife

clasp-knife; couteau (à cran d'arrêt), achille, eustache, jésus

jack-ladder (Naut. 1840)

one with wooden steps and side ropes; échelle de corde, échelle de Jacob

Jack-Nasty-Face

cf. Fanny 1 (1820-70)

cf. Jack 1 (18-19th)

dirty fellow (19-20th, ob.); sale individu, affreux jojo

ugly man; homme laid

Jack of all trades (17-20th)

one who (thinks he) can do everything; homme à tout faire, bricoleur, Maître Jacques, Jean-fait-tout

Jack of legs (1770-1890) very tall man; homme de grande taille

Jack-o'-lantern

will-o'-the -wisp (1673); feu follet

Halloween pumpkin-lantern (US); potiron-lanterne de Halloween

Jack Poke (US, NC, 20th) slowcoach; lambin

Jack-pudding (1648, ob.) (cf. merry-andrew 2)

Jack Randall (RS, 1859) (cf. Harry Randall)

Jack Robinson (NC, 19-20th, ob.) (cf. Abraham)

Jack Sauce (1560-1750) impudent fellow; insolent

Jack Shit, jack shit (US, NC, 20th) good-for-nothing, bon à rien nonsense; sottises, balivernes

Jack Snip (NC, 19-20th, ob.) (bad) tailor; (mauvais) tailleur

jack-snipe (Orn., 1663) a small species of snipe; petite bécassine

Jack Sprat (16-20th) a little fellow; petit bout d'homme, Tom Pouce

Jack Straw

man of straw $(1565, \mathrm{ob}$.$) ; homme de paille$ nonentity (1590-1910); homme insignifiant, nullité

Jack Straw's castle (NC, 19th) (cf. Fanny 1)

Jack Surpass (RS, 1851) glass (of sthg); verre (de qqch)

Jack Tar

cf. Jack 1 (1781)

bar (RS, 20th); bar

Jack the Dandy (RS, 20th)

cf. Charles Randy randy; émoustillé

Jack-the-lad (US, NC) show-off; m'as-tu-vu

Jack the Ripper (RS, 19-20th) kipper; hareng doux fumé a Jack of all trades is master of none, a Jack of all trades is of no trade

to be a dabbler in everything is no good; qui est propre à tout n'est propre à rien

all work and no play makes Jack a dull boy distractions are necessary; il faut aussi se distraire

before you can say Jack Robinson (18-20th) very quickly; très vite

cheap Jack (cf. cheap Charley)

every Jack has his Jill

there's a woman for every man; à chacun sa chacune

every man Jack (of them) (1840) everybody; tout le monde sans exception

fat Jack of the bone house (1850-1910) very fat man; homme très gros

flash Jack (1934) cf. Jack-the-lad cf. Jack Brag

for every Jack there is somewhere a Jill that can do with poor Jack about as she will

like pot, like lid; il n'est si méchant pot qui ne trouve son couvercle

to get Jack in the orchard (19-20th)

(cf. to introduce Charley)

gunnery Jack (Naut., 19-20th) gunnery lieutenant; lieutenant d'artillerie

handy Jack (19-20th) (cf. handy Andy)

high, Jack! (US, NC, 1920, ob.) hands up !; les mains en l'air !

the house that Jack built $(1860$, ob. $)$ prison; prison

if Jack's in love, he's no judge of Jill's beauty love is blind; l'amour est aveugle

to jack around (NC, 20th)

(cf. to dick around)

to jack s.o. around (NC, 20th)

to chaff s.o.; taquiner qqn

to badger s.o.; importuner qqn

to jack off (NC, 20th)

(cf. to Levy and Frank)

jumping-jack

cf. Jack-in-the-box 4

click-beetle (Ent., US); scarabée à ressort

sea-gull (Orn., 1896); mouette

on one's Jack (RS, 20th)

alone, without assistance; tout seul, sans aide

spring-heeled Jack (Naut., 19-20th)

racket-propelled torpedo; torpille-fusée

there are more Jacks than one at the fair

the same name is borne by many; il y a plus d'une personne qui porte le même nom, il y a (à la foire) plus d'un âne qui s'appelle Martin

whiskey Jack (Orn., 18th)

common green jay; geai commun

JACKY

Jacky

gin (NC, 1784); gin

Turk (Mil., 1915, ob.); Turc 
to sit up like Jacky (1930)

to sit up straight, to be on one's best behaviour; bien se tenir, bien se conduire

JACOB

Jacob

cf. silly Billy (1810-60)

ladder (18-20th); échelle

cf. Abraham (19th)

cf. whiskey Jack (18-19th)

Jacob's ladder

heavenly ladder (Bib.); échelle céleste, échelle de

Jacob

cf. jack-ladder

Greek valerian (Bot.); polémonie bleue, échelle de

Jacob

ladder in stocking (1859, ob.); échelle (dans un bas)

cf. Fanny 1

Jacob's shell (Moll.)

scallop-shell; pecten, coquille Saint-Jacques

Jacob's staff

cross staff; équerre d'arpenteur

cf. Aaron's rod 1 (Bot.)

Jacob's sword (Bot.)

JAKE

yellow iris; iris des marais

Jake

cf. Jack 2

methylated spirits (NC, 1920); alcool méthylique drunkard (US, NC); ivrogne

honest man (US, 1925); honnête homme

country jake (US)

(cf. Bill Shears)

JANE

Jane

sovereign (NC, 1860-1910); pièce de 20 shillings girl, woman (1915); fille, femme, Julie, nénette girlfriend (1922); bonne amie, Julie ladies' toilet; toilette pour dames

Jane Shaw (RS, 1930)

(cf. anytime Annie)

Jane Shore

cf. Fanny Adams (20th)

cf. anytime Annie (RS, 20th)

aunt Jane

cf. Jane 4

black woman who adopts a white lifestyle (US,

20th); femme noire qui adopte le style de vie des

blanches

Calamity Jane (US, 20th)

croaker; broyeuse de noir

good-time Jane (US, NC)

(cf. anytime Annie)

Lady Jane (1840)

(cf. Fanny 1)

Margery Jane (1900-1920)

(cf. Maggie Ann)

Mary Jane

cf. Fanny $1(1840$, ob. $)$

cf. Mary Ann 3 (US, NC, 20th)

plain Jane ill-favoured girl or woman; laideron

JANET

brown Janet (Naut., 19-20th, ob.)

knapsack; sac d'ordonnance, Azor

JASPER

Jasper (US, NC)

pious person (1926); personne pieuse

cf. Bob 1 (1953)

cf. Bill Shears (20th)

cf. Amy John (20th)

JEFF

Jeff

cf. Bob 1 (19-20th)

boring fellow (US, NC, 20th); raseur, Jean qui de tout se mêle

Mutt and Jeff (RS, 20th)

deaf; sourd

JEMIMA

Jemima

made-up tie (1899); nœud de cravate tout fait

cf. Ellen Terry (19-20th, ob.)

servant girl $(1887, \mathrm{ob}$.$) ; servante$

JEMMY

Jemmy

cf. Algy (1752-1900)

cf. Benjamin 3 (1830-1910)

burglar's crow-bar (1811); pince-monseigneur,

Charlotte, Jacques, Jacquot

Jemmy o'Goblin (RS, 1895)

sovereign; pièce de $20 \mathrm{sh}$.

all jemmy ! (1860-1910)

(cf. all my eye and Betty Martin !)

Jemmy Squaretoes (Naut., 19-20th)

(cf. old Davy)

JENNY

Jenny

cf. Jemmy 3 (17-19th)

hot-water bottle $(1880, \mathrm{ob}$.$) ; bouillotte$

she-ass (1808-20th); ânesse

wren (Orn., 1808-20th); roitelet

aircraft (US, NC); avion

spinning machine (Tex., 1796); machine à filer,

jeannette

cf. silly Billy (20th)

cf. Mary Ann 1

cf. anytime Annie (1946)

Jenny Hills (RS, 19-20th)

pills; pilules

cf. Charleys 2

Jenny Lea (RS, 19-20th)

cf. Betty Lea

flea; puce

Jenny Linda (RS, 1857)

window; fenêtre

jenny owl (Orn., 17th)

female owl; hibou femelle

jenny robin (Orn., 17th)

female redbreast; rouge-gorge femelle

Jenny Willocks (19-20th)

cf. Mary Ann 1

cf. Ethel 2 


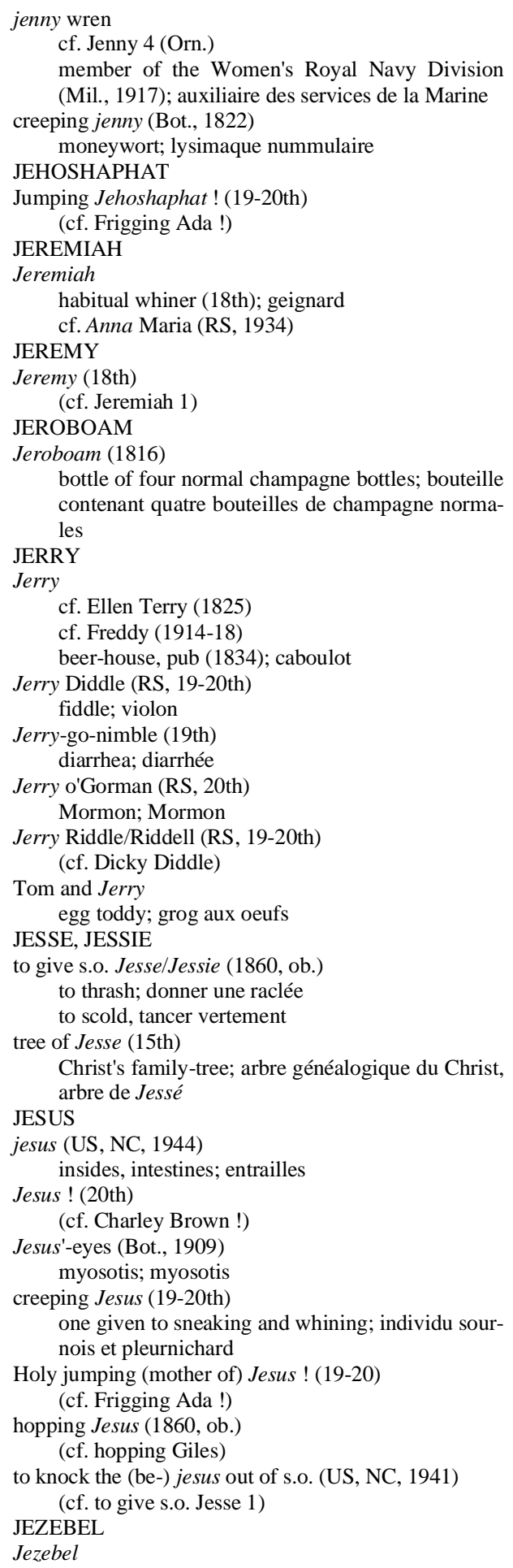

shrewish woman (16th); femme acariâtre, femme à $J o b$

JILL

cf. Abraham (19-20th)

Jill

cf. Jane 2 (1945)

female ferret; furet femelle

every Jack has his Jill

(cf. Jack)

for every Jack there is somewhere a Jill

(cf. Jack)

if Jack's in love, he's no judge of Jill's beauty

JIM (cf. Jack)

Jim Brown (RS, 1893)

town; ville

Jim Crow

cf. Bob $1(1850$, ob. $)$

cf. Jemmy 3 (1863)

negro (US, 1725); nègre

Jim Dandy (US, NC, 1880)

(cf. Daisy 2)

Jime Mace (RS, 19-20th)

face, visage

Jim Prescott (RS, 20th)

(cf. Charley Prescott)

JIMMIE

Jimmie (US, NC, 1955)

motor or car built by the GMC group; moteur ou voiture construits par le groupe GMC

JIMMY

Jimmy

cf. Jemmy 3 (19th)

immigrant (1859-97); immigrant

Jimmy Britts (RS, 20th)

(cf. Jerry-go-nimble)

Jimmy dog (US, NC, 20th)

marihuana cigarette; cigarette de marijuana

Jimmy Grant (RS, 1850)

(cf. Jimmy 2)

Jimmy hat (US, NC, 20th)

condom; préservatif, marguerite, imperméable à Popaul

Jimmy Riddle (RS, 19-20th)

(cf. Dicky Diddle)

Jimmy Skinner (RS, 1896)

(cf. Charley Skinner)

Bo Jimmy (US, NC, 20th)

(cf. Mary Ann 3)

dismal Jimmy (19-20th)

kill-joy; rabat-joie

to have a Jimmy Riddle (RS, 19-20th)

(cf. to Dicky Diddle)

JOAN

Joan (16th)

female rustic; paysanne, rustaude, Goton

Joan is as good as my lady in the dark when the candles are away, all the cats are gray; la nuit, tous les chats sont gris

Joan of Arcs (RS, 1945)

sharks; requins 
bobbin and Joan (Bot.)

lords and ladies; arum tacheté

Darby and Joan

cf. Edwin and Angelina

telephone (RS, 19-20th); téléphone

cf. Jack Jones

homely Joan (17-18th) coarse, ordinary woman; femme vulgaire

John-and-Joan (18-19th) (cf. Mary Ann 1)

on one's Darby and Joan (RS, 20th) (cf. on one's Jack)

JOANNA, JOHANNA

Joanna, Johanna (RS, 1912)

JOB piano; piano

$\operatorname{Job}(1846$, ob. $)$ henpecked husband; mari mené par sa femme

$J o b$ 's comforter (16th) one who aggravates distress; consolateur pessimiste, ami de $J o b$

$J o b$ 's news (16th) news of disaster; nouvelles de malheur

$J o b$ 's tears (Bot.) a species of grass (Coix lacrima); larmille, larmede-Job

Job's wife (19th) (cf. Jezebel 1)

as poor as $J o b$ ('s cat/turkey) (1820-1910) utterly poor; extrêmement pauvre, pauvre comme $\mathrm{Job}$

to have the patience of $J o b$

to be very enduring; être d'une grande patience, avoir la patience de $J o b$

laid up in $J o b$ 's dock (18-19th, ob.) in hospital for a venereal disease; hospitalisé pour une maladie vénérienne

\section{JOCK}

Jock

Scotchman (1870); Écossais

Scottish soldier (1914-18); soldat écossais

to look like Jock Blunt (18-19th)

JOE

to look disappointed; avoir l'air dépité

Joe, joe

cf. Sir Harry (1850)

cf. Bob 1

soldier (1940-45); soldat

coffee (US, NC, 20th); café

to joe (US, NC, 19-20th)

(cf. to Dicky Diddle)

Joe Beeswax ! (US, 19-20th)

(cf. Frigging Ada !)

Joe Blake (RS)

cake (19-20th); gâteau

cf. Ben Flake (1933)

snake (20th); serpent

delirium tremens, the shakes (20 th); delirium tremens

Joe Bloggs (US, NC, 20th)

Mr. Everyman; M. Tout-le-Monde
Joe Blow (NC)

cf. Joe Bloggs

cf. Bob 1

cf. Jack Brag

cf. Gabriel

recruit (1945); recrue

Joe Brown (RS, 1893)

(cf. Jim Brown)

Joe Buck (RS, 20th)

(cf. to Adam and Eve it)

Joe Doakes (US, NC)

So-and-so; Untel, Tartempion

Joe Erk (RS, 1917)

(cf. silly Billy)

Joe Goss (RS, 1910)

boss; patron

Joe Gurr (RS, 1930) stir; prison

Joe Hook (RS, 20th)

book; livre

crook; escroc

Joe Hunt (RS, 20th)

cf. silly Billy

wretched man; pauvre type

Joe Marks (RS, 1945)

(cf. Joan of Arcs)

Joe Miller (1816)

stale joke; plaisanterie éculée

Joe o' Gorman (RS, 19-20th)

foreman; contremaître

Joe-pye-weed (Bot., US)

kidney root; eupatoire pourprée

Joe Rocks (RS, 20th)

socks; chaussettes

Joe Ronce (RS, 1910)

(cf. Alphonse)

Joe Rook (RS, 20th)

(cf. Joe Hook)

Joe Rourke (RS, 19-20th)

fork, pickpocket; pickpocket

Joe Savage (RS, 1859)

cabbage; chou

Joe Skinner (RS, 19-20th)

(cf. Charley Skinner)

Joe Soap

cf. Alec (RS, 1930)

cf. Joe Bloggs

Joe Tank (RS, 20th)

bank; banque

Joe Trots (US, 19-20th)

(cf. Jerry-go-nimble)

Joe-wad (US, 19-20th)

toilet paper; papier de toilette

brown Joe ! (RS, 1855, ob.)

no !; que non!

cloudy Joe (Mil., 1940)

weather officer; officier météo

good Joe (US)

decent sort; brave type

have a go, Joe! (1935) 
go at it !; du cran, vas-y !, vas-y, Léon !, vas-y, Paulo!

holy Joe (NC)

army chaplain (Mil., 1900); aumônier militaire priest (1945); prêtre

prudish person (20th); pudibond

not for Joe ! (1867)

(cf. Fanny Adams! 2)

not to see the Joe Miller of it (1830-95)

not to see the joke; ne pas voir la plaisanterie

old Joe (US, NC, 1910)

(cf. Jack-in-the-box 6)

rolling Joe (1830-90)

smartly dressed fellow; gandin

sloppy Joe (US, NC, 1940)

loose-fitting sweater; pull-over lâche

sloppy Joe's (US, NC, 1940)

cheap eating-place; gargote

Uncle Joe (1942)

Joseph Staline

way to go, Joe ! (20th)

(cf. nice one, Cyril !)

JOHN

John, john (NC)

cf. Sir Harry 1 (1735)

Chinese (1870); Chinois

cf. Bob 1 (1906)

cf. bobby (1916)

cf. Joe Blow 1 (US, 1936)

boy friend (20th); bon ami, Jules

harlot's patron (1945); client d'une prostituée

cf. Abraham (20th)

John-a-dreams

dreamy fellow; rêveur, être logé chez Guillot le songeur

John -and-Joan

(cf. Joan)

John Barleycorn (17-20th)

(cf. Charley Frisky)

John Bates (US, NC)

(cf. Alec)

John Bluebottle (20th)

(cf. bobby)

John Blunt (1898)

(cf. Jack Blunt)

John Bull (1772)

Englishman; Anglais, John Bull

England; l'Angleterre, John Bull

John Chinaman

(cf. John 2)

John Citizen

the man in the street; M. Tout-le-Monde

John Cotton (RS, 1840)

(cf. Dolly Cotton)

johndarm (1917)

(cf. bobby)

John Davis (NC, 1926)

(cf. Jack 7)

John Doe

(cf. Bob 1)

John dogface (US, NC, 1940-45) (cf. Joe Blow 4)

John Dory (Icht., 1754)

dorado; dorade, Jean-doré

John Family (US, NC, 20th)

professional thief; voleur professionnel, Charles grafter; politicien véreux

John-go-to-bed-at-noon (Bot.) goat's beard; salsifis sauvage

John Hall (US, RS, 20th) alcohol; alcool

John Hancock, John Henry (US, NC) signature; signature

John Hop (RS, 1932, ob.) (cf. bobby)

John Law (US, NC, 1892) the police; la police

John o'Groat (RS, 1800) coat; manteau

John Q. Public (US, NC)

(cf. Joe Doakes)

John Roscoe (US, NC, 1935)

(cf. Betsy 1)

John Thomas (NC)

cf. Abraham (1840)

cf. Jack 4 (1860-1910)

John Willie (NC, 1910)

(cf. Abraham)

cheap John

(cf. cheap Charley)

dear John (letter) (US, Mil., 1940-45)

breaking-off letter; lettre de rupture

Donny John (19-20th)

Don Juan; Don Juan

dumbjohn (US, NC)

(cf. silly Billy)

to go to the john

(cf. to go to sir Harry)

honest John (US, 1943 GB) (cf. Jake 4)

long John (US, NC, 20th)

cf. Abraham

cf. Jack-of-legs

Sir John (19-20th)

(cf. Sir Harry 1)

square John (US, NC, 1930)

cf. Jake 4

cf. holy Joe

St. John's bread (Bot.)

fruit of the carob-tree; fruit du caroubier, pain de Saint-Jean

St. John's dance (cf. Guy)

St. John's fire

bonfire (of Midsummernight); feu de joie (du 24 juin), feu de la Saint-Jean

St. John's wort (Bot.)

(cf. Aaron's beard)

tarry-John (1888-1930)

(cf. Jack 1)

whiskey John (18th)

(cf. whiskey Jack) 
JOHNNIE, JOHNNY

Johnnie, Johnny

cf. Bob 1 (1670, ob.)

cf. bobby (1850)

cf. Sir Harry 1 (1880)

cf. Algy (1880)

penguin (1898); manchot

cf. Jacky 2 (Mil., 1914-18)

Greek (Naut., 19-20th); Grec

cf. John 2 (19th)

cf. Abraham (19th)

Johnny Armstrong (1920)

manual work; travail manuel hand-power; force des bras

johnnie bag (NC, 20th) (cf. jimmy hat)

Johnny-cake (18th)

maize or wheat meal cake; galette de farine de maïs ou de froment

Johnny cocks (Bot.) Orchis mascula

Johnny-come-lately (NC, 1850) newcomer; nouveau venu upstart; parvenu

Johnny Cotton (RS, 20th) (cf. Dolly Cotton)

Johnny Crapose (19-20th) Frenchman; Français

Johnny Head-in-the-air scatterbrains; tête en l'air

Johnny Horner (RS, 1909)

cf. Jack Horner

round the corner, i.e. at the pub; au cabaret

Johnny jumper, Johnny jump-up (Bot.) pansy; pensée, violette tricolore

Johnny Newcome

new-born child (1830, ob.); nouveau-né unexperienced youth (1850, ob.); novice

Johnny-on-the-spot (US, 1895)

one alert to his opportunities or present when needed; opportuniste

Johnny Randall (RS, 1860-1900) (cf. Harry Randall)

Johnny Raw $(1813$, ob.)

cf. Johnny Newcome 2

cf. Joe Blow 4

Johnny Ronce (RS, 1938)

(cf. Alphonse)

Johnnie Rutter (RS, 1880)

(cf. Dan Tucker)

Johnny Squarehead (Mil., 1914-18) (cf. Freddy)

Johnny Whop-Straw (19-20th) (cf. Bill Shears)

blue johnnies (19th) (cf. Joe Blakes)

jingling johnnie (Mus.) Chinese pavilion; chapeau chinois

rubber johnnie (NC, 20th)

(cf. jimmy hat)

to see Johnny (US, 20th)

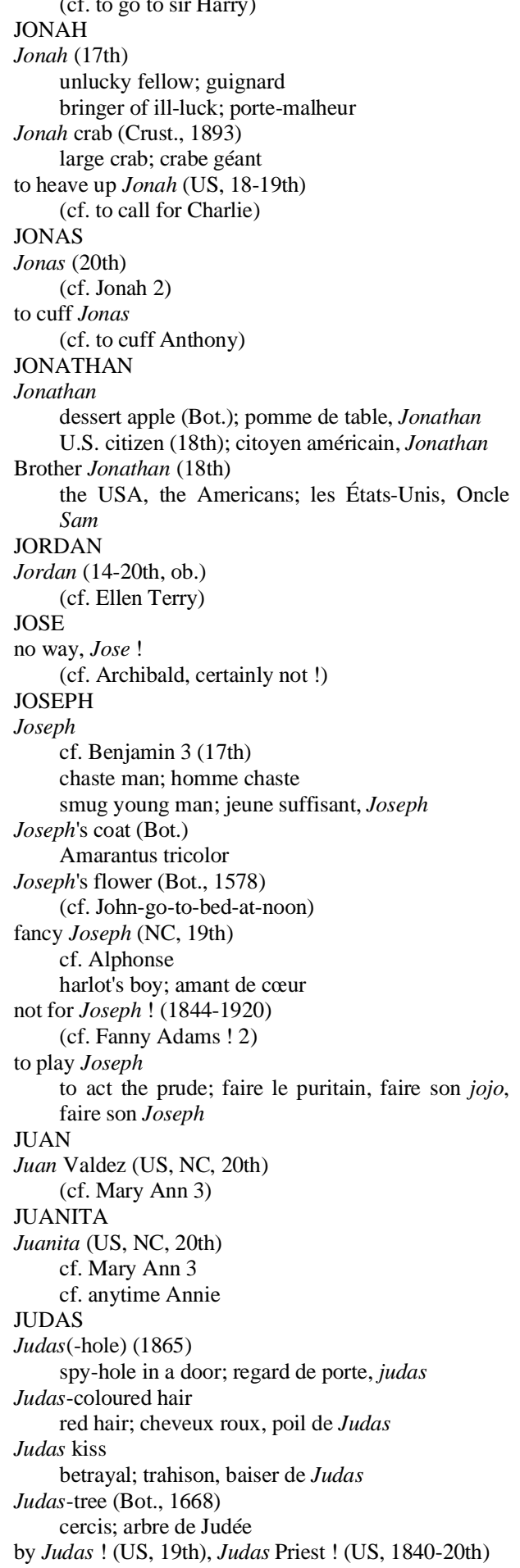




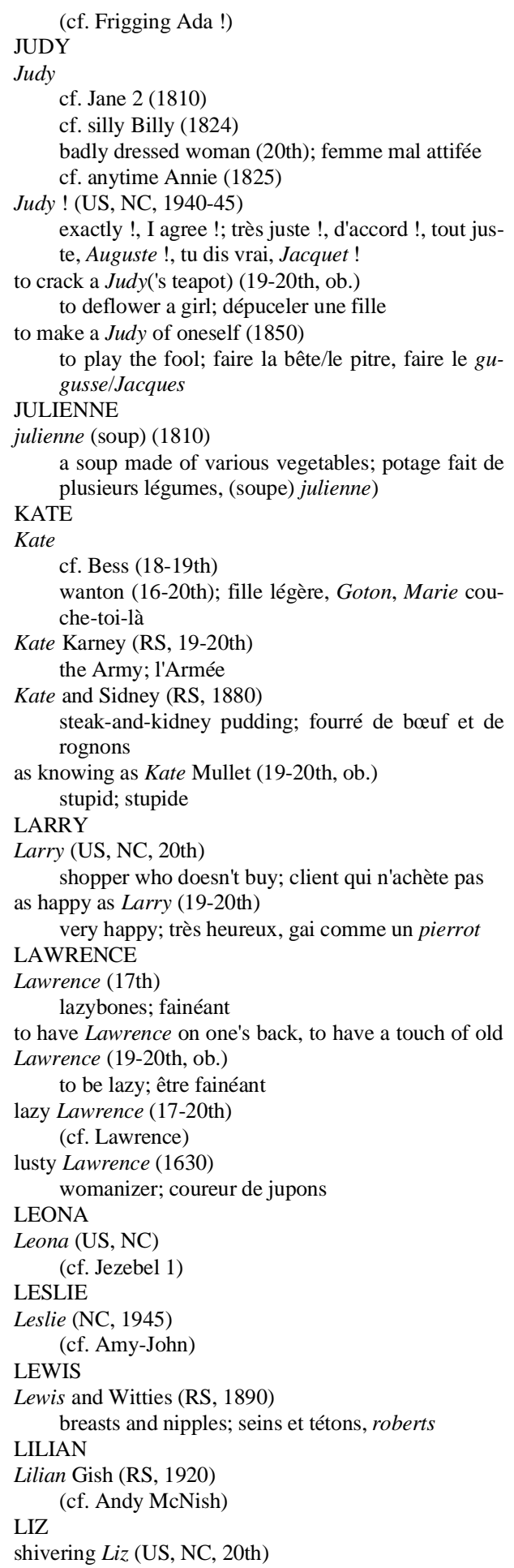

gelatin dessert; dessert à la gélatine; poitrine de Tante Rosalie

LIZA

Liza (US, 19-20th)

young black woman; jeune noire

he's saving them all for Liza! (1900, ob.)

said of a young man who will not use oaths or strike blows; se dit d'un jeune homme qui ne jure pas et ne frappe pas

outside, Liza ! (1880-1906)

be off !; allez, ouste !

LIZZIE

Lizzie (NC)

(cheap) car (1920); voiture (à bon marché)

(cheap) Lisbon wine (1920); petit vin portugais

cf. Amy-John (1928)

cf. Ethel 2 (US, 1928)

red Lizzie

(cf. Lizzie 2)

skinny Lizzie

skinny girl or woman; maigrichonne

tin Lizzie (1917, ob.)

cf. Henry

old car; vieille voiture

LOT

Lot's wife (19-20th)

salt; sel

as salt as Lot's wife backbone (1909) extremely salt; très salé

LOUIE

Louie (US, NC)

lieutenant (Mil., 1914-18); lieutenant migratory worker (1948); travailleur migrant

brass Louie (US, Mil, 1914-18) second-lieutenant; sous-lieutenant

to hang a Louie (US, NC, 20th)

to turn left; tourner à gauche

screwy Louie (US, NC, 20th)

(cf. Johnny Head-in-the-air)

LOUIS

Louis (1935)

(cf. Alphonse)

LOUISA

herb Louisa (Bot.)

lemon(-scented) verbena; verveine citronnelle

LUCILLE

Lucille (US, NC, 20th)

amphetamine; amphétamine

LUCY

Lucy (US, 19-20th)

(cf. Abraham)

Lucy in the sky with diamonds (US, NC, 20) LSD; LSD

Lucy Locket (RS, 20th) (cf. Davy Crockett)

sweet Lucy (US, NC, 20th)

(cf. Mary Ann 3)

LUKE

St Luke's bird (1780-1850)

ox; bœuf

St. Luke's summer 
last fine days around October 9th; derniers beaux LULU jours autour du 9 octobre, été de la Saint-Denis

lulu

gonorrhea (US, NC, 20th); blennoragie

breast (US, 20th); sein, robert

cf. sir Harry

to see Lulu (US, 20th)

(cf. to go to sir Harry)

MADGE

Madge

cf. Fanny 1 (18th)

cf. anytime Annie (US, 19-20th)

MAGDALEN

Magdalen

reformed prostitute (1693); fille repentie, Madelei-

ne

kind of pear (Bot., 1706); sorte de poire, madeleine

MAGGIE

Maggie

cf. Betsy 1 (US, NC, ob.)

dishonest peddler; colporteur marron

shot that hits the target (US, NC, Mil., 1884); tir au but

Maggie Ann

(cf. Ann)

Maggie's drawers (US, Mil.)

missed shot; tir à côté de la cible

Maggie Moores (RS, 1942)

drawers; culotte (fém.)

to get it where Maggie wore the beads (1905-25)

to catch it, to get the blame; se faire attraper, se

faire appeler Arthur, se faire appeler Jules

\section{MAHOMET}

if the mountain will not to go to Mahomet, let Mahomet go to the mountain

to raise an opportunity that would not occur by itself; faire naître une occasion qui ne se présenterait pas d'elle-même, si la montagne ne vient pas à Mahomet, Mahomet ira à la montagne

MALCOLM

Malcolm Scott (RS, 20th)

hot; chaud

MARGARET

herb Margaret (Bot., 1640, ob.) (cf. Daisy)

MARGE

Marge (US, NC, 20th) (cf. Amy-John)

MARGERY

Margery (1850-1900) (cf. Ethel 2)

Margery Jane (cf. Jane)

MARIA

Maria Monk (RS, 19-20th) spirit, pluck, spunk; entrain, courage seminal fluid, spunk; sperme

Anna Maria

(cf. Anna)
Aunt Maria (1903)

(cf. Fanny 1)

Black Maria

prison van (1847); voiture cellulaire

heavy shell (Mil., 1914-1918); gros obus hearse (US, NC); corbillard

MARIE

Marie Corelli (RS, 20th)

television, telly; télévision

MARTIN

martin (Orn., 15th)

bird of the swallow family; oiseau proche des hirondelles, martinet

Martin dry (Bot., 1664)

a kind of pear; espèce de poire, martin-sec

Martinmas

November 11th; le 11 novembre, la Saint-Martin

Martinmas summer of love

the last flame of love; la dernière flambée d'amour

sand-martin (Orn.)

cotile; hirondelle de rivage

St. Martin's summer

days of fine weather about Martinmas; beaux jours autour du 11 novembre, été de la SaintMartin

Sir Martin Wagstaff (17th)

(cf. Colleen Bawn)

MARY

Mary (US, NC)

cf. Jane 2 (1945)

cf. Amy-John (20th)

cf. Mary Ann 1 (20th)

cf. Mary Ann 3 (20th)

Mary and Johnnie (US, NC, 20th) (cf. Mary Ann 3)

Mary Ann (cf. Ann)

Mary Blane (RS) cf. Andy Cain (1860-1910)

train (1880-1914); train

Mary Jane

(cf. Jane)

Mary-lily (Bot.)

Madonna lily; lis blanc

Mary Warner (US, NC, 20th)

(cf. Jimmy dog)

Mary Weaver (US, NC, 20th)

(cf. Mary Ann 3)

to be married to Mary Fist; to fuck Mary Fist (US, NC, 20th)

(cf. to Levy and Frank)

bloody Mary

tomato juice and gin (1944); jus de tomates et gin, bloody Mary

cf. curse of Eve (US, 20th)

I'm willing, but Mary isn't (20th)

my stomach won't stand it; mon estomac ne le permet pas

little Mary

cf. Fanny 1 (US, 20th)

stomach; estomac (1903) 
to kiss Mary (US, NC, 20th)

to smoke marihuana; fumer de la marijuana

\section{MATHURIN}

malady of St. Mathurin (17th, ob.)

madness; folie, colique/mal (de) Saint-Mathurin

MATILDA

to carry/waltz Matilda (1893)

MAUD

to carry one's swag; porter son baluchon

Maud (NC, 1930)

MEG

(cf. Mary Ann 1)

Meg

guinea $(1742$, ob.); guinée

hoyden $(1538, o b$.$) ; garçon manqué$

as long as Meg of Westminster (16-18th)

said of a very tall woman; se disait d'une femme de grande taille

long Meg (16-19th)

tall woman; grande perche

Mons $\mathrm{Meg}$

the great gun of Edinburgh Castle (1575); le canon

du château d'Édimbourg

cf. Fanny 1 (19th)

MELVIN

Melvin (US, NC, 1950)

(cf. Jeff 2)

METHUSELAH

as old as Methuselah (17-20th)

(cf. as old as Adam)

MICHAEL

Michaelmas

September 29th; le 29 septembre, la Saint-Michel

Michaelmas daisy (cf. Daisy)

to extract/take the Michael out of s.o. (NC, 20th) (cf. to jack s.o. around)

(hip) Michael, your head's on fire ! (18-19th) said to a red-haired man; quolibet à l'adresse d'un rouquin

MICK

Mick

Irishman (1872); Irlandais

Roman Catholic (US, NC, 1924); catholique

Mexican (US, 20th); Mexicain

cf. silly Billy

Mick O'Dwyer (RS, 19-20th)

(cf. Anna Maria)

mad Mick (RS)

pick (19th); pioche

cf. Abraham (1910)

old Mick (RS, 19-20th)

(cf. Bob, Harry and Dick)

Pat and Mick (RS)

thrashing, lick; raclée

prick (cf. Abraham)

MICKEY

Mickey

cf. Mick 1

potato (US, 1945); pomme de terre

cf. Abraham (19-20th) cf. Mickey Finn (US, NC, 1938)

Mickey Finished (US, 20th)

(cf. blind as Chloe)

Mickey Finn (US, 1930)

any drug given to an unsuspecting person; potion administrée à qqn à son insu

Mickey Mouse

house (RS, 1930); maison

vividly sentimental (adj., US, 1945); d'un sentimentalisme exagéré

cf. Fanny 1 (US, 20th)

easy (NC, 20th); facile

low-grade, useless (NC, 20th); de qualité inférieure, sans valeur

Mickey Mouse is kaput (US, 20th)

(cf. curse of Eve)

Mickey Mouse mattress (US, 20th)

(cf. fanny pad)

to take the Mickey out of s.o. (NC, 20th)

MIKE

(cf. to jack s.o. around)

Mike (1873)

(cf. Mick 1)

Mike and Ike

(cf. Ike)

Mike Bliss (RS, 19-20th)

(cf. Dicky Diddle)

Mike Malone (RS, 19-20th)

(cf. Jack Jones)

clever Mike (RS, 20th)

bike; vélo

for the love of Mike! for God's sake !; pour l'amour du ciel !

Pat and Mike (RS, 19-20th) (cf. clever Mike)

red Mike (US, NC, 20th) woman-hater; misogyne

to take the Mike out of s.o. (NC, 20th)

MOLLY

(cf. to jack s.o. around)

Molly

cf. Ethel (18th)

milksop (18th); poule mouillée

cf. anytime Annie (1719)

cf. Mary Ann 1 (1709)

molly-coddle (1833)

(cf. Molly 1 \& 2)

Molly cotton-tail (US)

common rabbit; lapin commun

Molly's hole (NC, 19-20th, ob.)

(cf. Fanny 1)

Molly Malone (RS, 20th)

(cf. Darby and Joan 2)

Molly O'Morgan (RS, 19-20th)

organ; orgue

Miss Molly $(1750, o b$.

(cf. Molly 1 \& 2)

MONA

Mona (RS, 1919)

female moaner; geignarde 


\section{MOSES}

Moses

meek, obedient man; individu humble et soumis leader (16th); guide, chef

Jewish money lender; prêteur juif

Moses! (1858, ob.), holy Moses! (1853), walking

Moses! (1920)

dear me!, bless my soul !; grands dieux !, par exemple !

Moses basket

basket cot; berceau-corbeille, moïse

(holy) jumping Moses ! (19-20th)

(cf. Frigging Ada !)

meek as Moses

meek as a lamb; doux comme un agneau

to be taken in like Moses at the fair

to give gold dust for glass beads; faire l'échange de l'Indien

to stand Moses (19-20th)

for a man, to adopt a child; pour l'homme, adopter un enfant

MURPHY

murphies (US, NC, 20th)

(cf. Lewis and Witties)

NANCY

Nancy

cf. Ethel 2-3 (19-20th, ob.)

cf. Mary Ann 1 (19-20th)

Nancy boy (19-20th)

(cf. Mary Ann 1)

Nancy Dawson (1887-1910)

(cf. Ethel 2)

Nancy Lee (RS)

cf. Jenny Lee 2 (1860)

cf. Jenny Lee 1 (20th)

ask my Nancy ! (1810-1910)

I don't know !; je n'en sais rien !

Miss Nancy

(cf. Nancy $1 \&$ 2)

to talk Miss Nancy (1910)

to mince; minauder, faire sa Julie

NANNY

Nanny

cf. anytime Annie (17-19th)

nurse (1864); nounou

she-goat (1890); biquette

Nanny berry (Bot., US)

wayfaring-tree; viorne cotonneuse

nanny-goat (1788)

(cf. Nanny 3)

to play the nanny-goat (1905)

(cf. to make a Judy of oneself)

Fanny-Nanny

(cf. Fanny)

NARCISSUS

Narcissus

male in love with his own beauty; homme épris de sa propre beauté, Narcisse

daffodil (Bot., 1548); genette, narcisse

poet's narcissus (Bot., 16th)

pheasant's eye; œillet de Pâques, jeannette
NEBUCHADNEZZAR

to take Nebuchadnezzar out to grass (19th)

(cf. to Adam and Eve it)

NED

Ned

cf. Meg 1 (1750-1890)

cf. Dicky 1 (1830)

ten-dollar gold piece (US, ob.); pièce d'or de dix dollars

Ned Kelly (RS, 1920)

belly; ventre

Ned Skinner (RS, 1900)

(cf. Charley Skinner)

Uncle Ned (RS, 19-20th)

bed; lit

NEDDY

Neddy

cf. Dicky 1 (17-20th)

cf. Meg 1 (1760-1850)

cf. silly Billy (1820)

life-preserver (NC, 1864); casse-tête

NELLIE

Nellie

giant petrel (Orn., 1823); pétrel géant

cheap wine; piquette

Nellie Bligh (RS, 20th)

eye; œil

Nellie Duff (RS, 1939)

puff, breath; souffle, vie

Nellie fag (US, NC, 20th)

(cf. Mary Ann 1)

nice Nellie

cf. holy Joe 3 (1952)

cf. Mary Ann 1 (US, NC, 1910)

not on your Nellie (Duff) ! (1939)

(cf. Archibald, certainly not !)

pussy Nellie (NC, 1910)

(cf. Mary Ann 1)

since Nellie had her operation (1910)

(cf. since Adam was a boy)

NELLY

Nelly (US, NC, 20th)

cf. Amy-John

cf. Mary Ann 1

aunt(ie) Nelly (RS, 19-20th)

(cf. Ned Kelly)

NESTOR

Nestor (1588)

a wise old man; vieillard plein de sagesse, Nestor

NICHOLAS

clerk of St. Nicholas (1570-1820)

highwayman; voleur de grand chemin

Saint Nicholas (16-19th)

(cf. old Davy)

NICK

old Nick (1668)

(cf. old Davy)

to have the cheek of old Nick

$\mathrm{NOAH}$

to be very impudent; être d'une grande insolence

Noah's ark 
small bivalve mollusc (Moll., 1713); arche cf. Benjamin 3 (1858-1920)

fun, lark (RS, 1887); amusement, plaisir dark (RS, 20th); sombre nark (RS, 1920) (cf. silly Billy) nark, informer (RS, 20th); indicateur (de police) Noah's boy (US, NC) ham, as served to be eaten; jambon servi en plat Noah's night-cap (Bot.) eschsholtzia; eschscholtzie (de Californie)

NORMA

Norma Snockers (RS, 20th) (cf. Lewis and Witties)

OLIVER

Oliver

the moon (1780-1900); la lune cf. Oliver Twist (1909)

Oliver Cromwell (RS, 20th) to understand, to tumble; comprendre

Oliver's skull (1690-1870) (cf. Ellen Terry)

Oliver Twist fist (RS, 19-20, ob.); poing twist; escroquerie

do you Oliver? (20th) do you understand ?; tu comprends ?

to give s.o. a Roland for an Oliver to pay s.o. back in his own coin; rendre à qqn la monnaie de sa pièce to put the Oliver on it (1910)

to act dishonestly; procéder de façon malhonnête OSCAR

Oscar

cf. Mary Ann 1 (19-20th)

Japanese plane (Mil., 1942); avion japonais Hollywood movie award; récompense cinématographique américaine, Oscar

Oscar Asche (RS, 20th) cash (money); argent liquide

OWEN

Owen Nares (RS, 1910) chairs; chaises, sièges

PADDY

Paddy, paddy

cf. Mick 1 (1748) rage, temper (1894); colère

Paddy-bird (Orn.) Java sparrow (1727); moineau de Java sheathbill (1894); chionis

Paddy's grapes (NC, 1905) (cf. Mickey 2)

Paddy Kelly (19-20th, ob.) (cf. bobby)

Paddyland, Paddy('s) land (1820) Ireland; l'Irlande

Paddy's lantern (19-20th) (cf. Oliver 1)

Paddy Quick (RS) thick $(1859$, ob.); gros stick (1859); bâton kick (1910); coup de pied
Paddy Rammer (RS, 19-20th) hammer, marteau

Paddy Whack, paddywhack (1881) (cf. Paddy 1 \& 2)

as Irish as Paddy's pig (1890) staunchly Irish; Irlandais bon teint

PAT

Pat

cf. Mick 1 (1825)

cf. John 2 (1923)

Pat and Mick (cf. Mick)

Pat and Mike (cf. Mike)

Patland (19-20th) (cf. Paddyland)

Patlander (1820)

(cf. Mick 1)

Pat Malone (RS, 20th) (cf. Jack Jones)

as Irish as Pat's pig (1890) (cf. as Irish as Paddy's pig)

on one's Pat (Malone) (1916) (cf. on one's Jack)

PATRICK

as Irish as Patrick's pig (1890) (cf. as Irish as Paddy's pig)

mother of St. Patrick (1811, ob.) (cf. Fanny 1)

St. Patrick (1650-1850) the best whisky; le meilleur whisky

PAUL

Paul's betony (Bot.) wood speedwell; thé d'Europe, véronique

Paul Pry (1825) nosy person; personne curieuse, fille d'Ève, Jean qui de tout se mêle

to borrow from/to rob Peter to pay Paul to borrow to pay old debts; faire un trou pour en boucher un autre, décoiffer/ découvrir/déshabiller Pierre pour coiffer/couvrir/habiller Paul

PEGGY

Peggy

pied wagtail (Orn., 1836); bergeronnette grise one-legged (beggar) (US, NC, 20th); (mendiant) unijambiste

\section{PENELOPE}

Penelope

virtuous woman (1581); femme vertueuse, Pénélope

a genus of gallinaceous birds in Central America (1605); espèce de gallinacé d'Amérique centrale, pénélope

PERCY

Percy

cf. Abraham (NC, 20th)

cf. Mary Ann 1 (1925)

studious, educated fellow (1940); esprit cultivé

Percy boy (US, NC, 1952)

(cf. Ethel 2)

Percy pants (US, NC, 1945) 
(cf. Ethel 2)

to point Percy at the porcelain (NC, 20th)

(cf. to Dicky Diddle)

silent Percy (Mil., 1914-18, ob.)

German high-velocity gun; canon à tir rapide allemand

PETE

Pete (US, NC, 1920)

safe; coffre-fort, Jacques, Jacquot

for the love of Pete

(cf. for the love of Mike)

sneaky Pete (US, NC)

any illegal alcoholic beverage (1940); toute boisson alcoolisée illégale

bad whisky (1958); whisky de mauvaise qualité cf. Nellie 2 (1952)

PETER

Peter

bundle, swag (17-19th); sac, baluchon

cf. Pete $(1915$, ob. $)$

cf. Abraham (19-20th, ob.)

(St.) Peter's bark/boat (Rel.)

the Roman Catholic Church; l'Église catholique

peter-cheater (US, NC, 20th)

(cf. fanny pad)

(St.) Peter's fish (Icht.)

haddock; aiglefin

Peter Grievous

fretful child (19-20th, ob.); enfant grognon croaker (1850); pessimiste

peter pansy (US, NC, 20th)

(cf. Mary Ann 1)

Peter's penny/pence (Rel., 1860)

voluntary contribution(s) of Roman Catholics to the papal treasury; contribution(s) volontaire(s) des catholiques au trésor papal, denier de saint Pierre

(St.) Peter's wort (Bot.)

cowslip; primevère, commune

feverfew; pyrèthre, pied d'Alexandre

blue Peter (Naut.)

flag of immediate sailing; pavillon de partance

herb Peter

(cf. Peter's wort)

to go/pass through St. Peter's needle (19-20th) PHIL

to get a sound thrashing; recevoir une bonne raclée

Phil McBee (RS, 1870-1940)

(cf. Jenny Lee 2)

PHILIP

Philip (NC, 1860, ob.)

(cf. bobby)

PHYLLIS

Phyllis (20th)

(cf. Jack-in-the-box 6)

PIERROT

Pierrot (1741)

a character of the Commedia dell'Arte and of the

French pantomime; personnage de la comédie italienne et de la pantomime française, Pierrot
POLL

Poll (1630)

parrot; perroquet, Jacquot

Pretty Poll

(cf. Poll)

POLLY

Polly (17th)

(cf. Poll)

Polly Flinder (RS, 19-20th)

(cf. Jenny Linda)

PONTIUS

since Pontius was a pilot (1944)

(cf. since Adam was a boy)

\section{RACHEL}

Rachel (US, NC, Mil.)

high gear on motor vehicle; vitesse supérieure d'un véhicule

RALPH

Ralph

cf. silly Billy (18th)

cf. Abraham (US, NC, 20th)

Ralph Spooner (17th)

(cf. silly Billy)

to call/cry Ralph (NC, 20th)

(cf. to call for Charlie)

to go to Europe with Ralph and Earl in a Buick (US,

NC, 20th)

(cf. to call for Charlie)

to hang a Ralph (US, NC, 20th)

to turn right; tourner à droite

to ralph (US, NC, 20th)

(cf. to call for Charlie)

to talk to Ralph on the big white phone (US, NC, 20th) (cf. to call for Charlie)

\section{REUBEN}

Reuben (US, NC, 1890); (cf. Billy Shears)

RICHARD

Richard (NC)

cf. Dick 6 (18-20th, ob.)

hunchback (1948, ob.); bossu

cf. Dick 4 (1952)

Richard's himself again! he's got over it !; le voilà retapé !

Richard Roe

(cf. John Doe)

Richard the Third (RS) bird (19-20th); oiseau

turd (19-20th); étron

word (20th); mot

every Richard (US, 20th)

(cf. Dick, Tom and Harry)

to get the (ripe) Richard (1915, ob.)

to get hissed; se faire huer

to get the cold shoulder; se faire snober

Poor Richard's sayings (18th)

advice prompted by common sense; conseils de bon sens, la science du Bonhomme Richard

Rob Roy (RS, 1800-1930)

boy; jeune garçon 


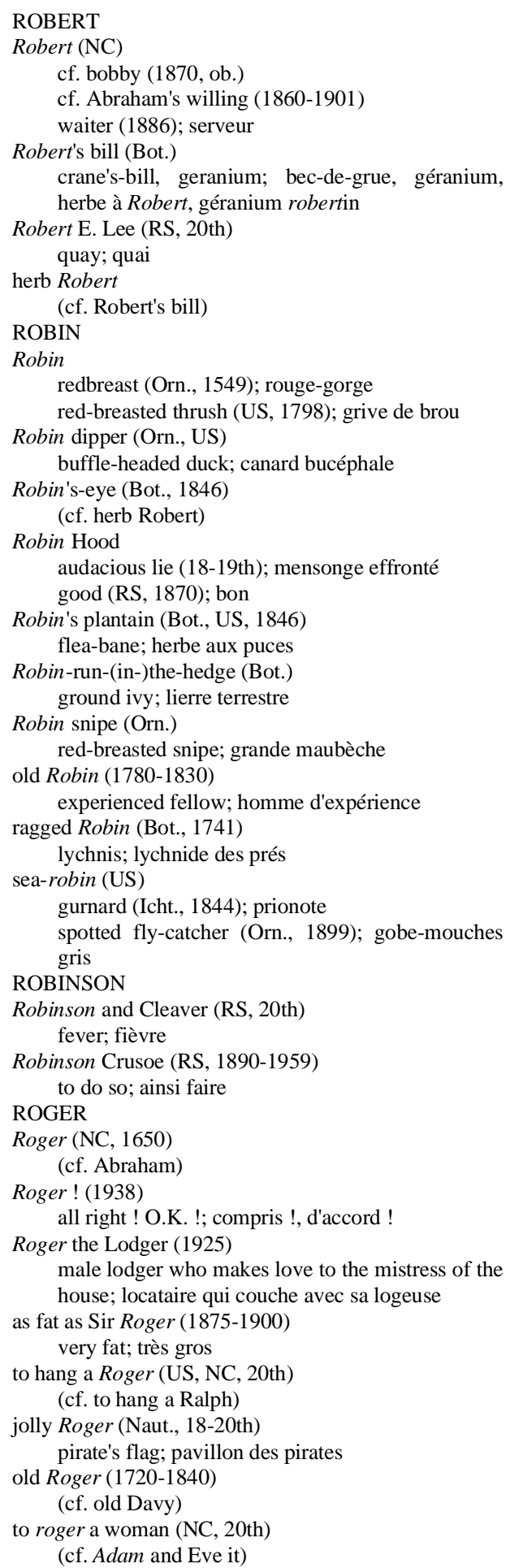

ROLAND

to give s.o. a Roland for an Oliver (cf. Oliver)

ROSALIE

Rosalie (Mil., 1914-1918) bayonet; baïonnette, Charlemagne, Rosalie

ROSEMARY

it's Rosemary Lane to a rag shop (1810-90) it's long odds; les chances sont minimes

ROSIE, ROSY

Rosy Lee (RS, 19-20th) (cf. Jenny Lea 1)

Rosie Loader (RS, 20th) whisky and soda; whisky-soda

RUBE

Rube (US, NC) cf. Billy Shears (1890) cf. silly Billy (1900)

RUFUS

Rufus (19-20th, ob.) (cf. Fanny 1)

RUPERT

Rupert (NC, 1908) (cf. Abraham)

Prince Rupert's ball/drop/tears (Phys.) glass beads; larmes bataviques

Prince Rupert's metal an alloy of copper and zinc; alliage de cuivre et de zinc, métal du prince Robert

RUTH

Ruth (US, NC, 20th) (cf. Jane 4)

to call/cry Ruth (NC, 20th) (cf. to call for Charlie)

SALLY

Sally Ann (cf. Ann)

Sally Lunn light tea-cake containing currants; petit pain aux raisins

Aunt Sally SAM a game played at fairs; jeu de massacre (à la foire)

Sam (1843) (cf. silly Billy)

Sam Brown (Mil.) cross-belt; baudrier officer (1940-45); officier

Sam Hill (1830-20th) hell; enfer cf. old Davy

to stand Sam

to pay for drinks (1823); payer la tournée to promise a person sthg (20th); promettre qqch à qqn

Uncle Sam (1812) (cf. Brother Jonathan)

Uncle Sam's party (US, NC, 1945) payday for federal employees; jour de paie des fonctionnaires upon my Sam ! (1879) 
upon my word !; parole d'honneur ! what the Sam Hill !

SAMMY

(cf. Frigging Ada!)

Sammy

cf. silly Billy $(1830$, ob.

cf. Abie (US, 20th)

US soldier (1917); soldat américain

Sammy Soft $(1840$, ob.)

(cf. silly Billy)

simple Sammy

(cf. silly Billy)

SAMSON

Samson's post (Naut., 16th) stranchion; épontille (à manche)

a veritable Samson (16th)

a very strong man; homme d'une grande force, un vrai Hercule

SANDY

Sandy (15th)

(cf. Jock)

Sandy McNab

taxicab (RS, 1946); taxi

strike breaker (RS, 1920); briseur de grève

SARAH

Sarah Gamp (RS, 20th)

lamp; lampe

Sarah Soo (RS, 20th)

(cf. clipped Dick)

SHEILA

Sheila, sheila (NC, 19-20th)

(cf. Jane 2)

SIDNEY

Kate and Sidney (cf. Kate)

SILAS

Silas Hocking (RS, 19-20th) stocking; bas

SIMON

Simon

sixpence (NC, 17-19th); pièce de six pence dollar (US, 1859, ob.); pièce d'un dollar

simple Simon

cf. silly Billy

diamond (US, NC, 1932); diamant

the real Simon Pure (1859)

the real, genuine person or thing; le vrai, l'authen-

SIS

tique (personne ou chose)

cousin Sis (RS, 20th)

(cf. Dicky Diddle)

SOLOMON

Solomon's seal (Bot., 1543)

Polygonatum vulgare; muguet anguleux, sceau de Salomon

the British Solomon

James I; Jacques $\mathrm{I}^{\mathrm{er}}$ d'Angleterre

justice of Solomon (1554)

fair judgment; jugement équitable, jugement de Salomon

wisdom of Solomon (1554) great wisdom; grande sagesse, sagesse de Salomon

to be no Solomon not to be very intelligent; n'être pas très intelligent to do a Solomon (20th)

to pretend to be very wise; jouer au sage

SOPHIA

herb Sophia (Bot.)

Descuraina sophia

STEPHEN, STEVEN

Stephen, Steven (NC, 1810-50)

(cf. Jack 7)

even-Steven (1942)

share and share alike; en parts égales, équitablement

to call it even-Steven (20th)

to call it quits; faire quitte

STEVE

believe me, Steve! (20th)

you can take it from me !; tu peux me croire !

come on, Steve ! (1925)

hurry up !; dépêche !

got me, Steve ? (1914)

(cf. do you Oliver?)

STEVIE

take it easy, Stevie !

easy does it !, relax !; doucement !, du calme !, à l'aise Blaise !, relax Max !, cool Raoul !

\section{SUSAN}

black-eyed Susan (US)

yellow daisy; marguerite américaine

Black-eyed Susan State (US)

the State of Maryland; le Maryland

lazy Susan

revolving board; plateau tournant

silent Susan (Mil., 1914-18)

SUSIE

(cf. silent Percy)

Susie

cf. Simon 1 (NC, 1932)

woman doing Army work (Mil., 1915-18); auxiliaire féminine

Sister Susie

(cf. Susie 2)

TAFFY (17-20th)

Taffy (1682)

Welshman; Gallois

TED

Ted

cf. Freddy 1 (Mil., 1943-45)

cf. Teddy boy

cf. Teddy bear 1 et 2

TEDDY

Teddy (US, NC)

cf. Mick 1 (1900)

woman's undergarment (1920, ob.); sous-vêtement de femme

Teddy bear

stuffed plush bear (1907); ours en peluche, ours

Martin

fur coat (Mil., 1915); manteau de fourrure 
Teddy boy (1950) hooligan; jeune voyou

TERRENCE

to train Terrence on the terracotta (NC, 20th) (cf. Dicky Diddle)

\section{THOMAS}

Thomas doubting, sceptic fellow (1883); un incrédule, un sceptique, un saint-Thomas cf. Abraham (19-20th) cf. Jack 4 \& Robert 3

Thomas Tilling (RS, 20th) (cf. Abraham's willing)

John Thomas (cf. John)

man Thomas (17-19th) (cf. Abraham)

to sup with Thomas Gresham (17th) (cf. to dine with St. Anthony)

tickle-Thomas (19-20th)

TILLY

(cf. Fanny 1)

Tilly Bates (RS, 20th)

late; tard, en retard

TIMOTHY

Timothy (1847)

TOM

(cf. Abraham)

Tom

cf. old Tom $(1823, \mathrm{ob}$.

cf. Abraham (19th)

cf. Jane 2 (20th)

cf. anytime Annie (19-20th)

negro (US, 19-20th); négro

cf. Ellen Terry

cf. Amy-John

Tom and Jerry

(cf. Jerry)

Tom-a-Styles (1770, ob.)

(cf. John Citizen)

Tom and funny (RS, 1909, ob.)

(cf. Jack 7)

tomboy (16th)

(cf. Meg 2)

tom-cat (1791)

male cat; matou

Tom Cony (17-19th)

(cf. silly Billy)

Tom Cox (19-20th) shirker; carotteur, tire-au-flanc

Tom, Dick and Harry (cf. Dick)

Tom-doodle (18-20th, ob.) (cf. silly Billy)

Tom Farthing (cf. silly Billy)

tomfool, Tom Fool (1721) (cf. silly Billy)

Tom, Harry and Dick (cf. Dick)

Tom Long $(1630, \mathrm{ob}$. one long in a-coming or in telling a tale; personne qui met du temps à venir ou à raconter une histoire tom-noddy

cf. silly Billy (1828)

puffin (Orn., 1702); macareux

Tom Right (RS, 1857, ob.)

night; nuit, bernarde

Tom Sawyer (RS, 19-20th)

lawyer; avocat

Tom Tart

cf. Jane 3 (RS, 1942)

fart (RS, 20th); pet, louise

Tom Thacker (RS, 19-20th, ob.) tobacco; tabac

Tom Thumb

hero of a fairy tale (16th); le petit Poucet cf. Jack Sprat (16th)

cf. Jack Straw 2 rum (RS, 19-20th, ob.); rhum

Tom Thumb nasturtium (Bot., 1876) dwarf nasturtium; capucine naine

Tom Thumb umbrella short umbrella; parapluie court, tom-pouce tom-tit

blue titmouse (Orn., 1709); mésange bleue cf. Tom Thumb 2 (1741)

to tom-tit (RS, 19-20th)

(cf. to george)

Tom Tripe (RS, 1859-1900) pipe; pipe

Tom Tug (RS)

mug (cf. Alec) (1874)

bed-bug (1909); punaise

Tom Tyler (cf. Job)

any Tom, Dick or Harry (cf. Dick)

blind Tom (US, NC) baseball referee; arbitre de base-ball

Long Tom long-range gun (1865); canon à longue portée cf. Abraham (1898)

old Tom (1820, ob.)

(cf. Jacky 1)

peeping Tom (1837) voyeur; voyeur

there was nobody there but Tom, Dick and Harry there were only few people; il n'y avait que trois pelés et deux tondus

to be hail-fellow-well-met with Tom, Dick and Harry to consort with everybody; se lier avec tout le monde, frayer avec Pierre, Paul et Jacques

Uncle Tom (US, NC, 1956)

a Negro who defers to or imitates white people; Noir servile envers les Blancs ou qui les imite

TOMMY

Tommy

cf. silly Billy (1829)

British soldier (1893); soldat britannique, Tommy

cf. Abraham (19-20th)

cf. curse of Eve (19-20th) 


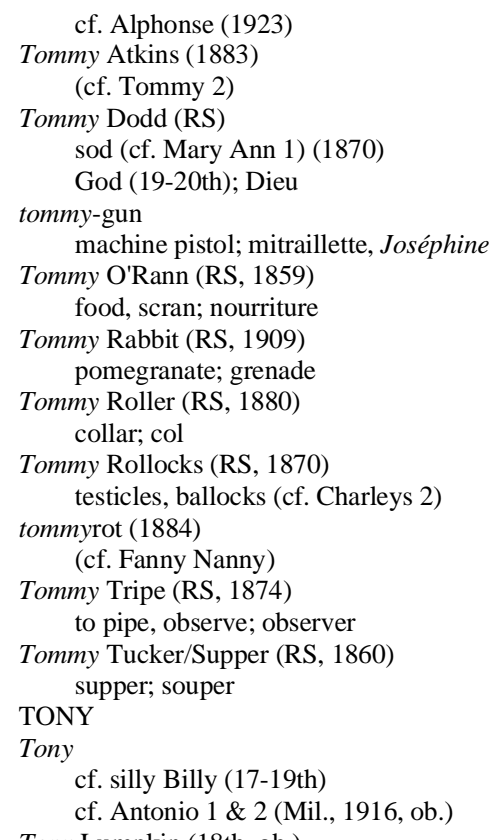

Tony Lumpkin (18th, ob.) clumsy, blundering fellow; empoté, maladroit, Fiacre

TOTTIE

tottie (1880)

high-class whore; demi-mondaine

Tottie fie (NC, 20th) young woman given to throwing her weight about; VERA poseuse, Marie-Chantal

Vera Lynn (RS, 1940)

(glass of) gin; (verre de) gin

(cf. Jacky 1)

VERONICA

Veronica

shroud (13th); suaire, véronique

VICTOR

cf. Paul's betony (Bot., 1527)

Victor Trumper (RS, 1905)

cigarette-end, bumper; mégot

VICTORIA

Victoria

a variety of plum (Bot., 1860); variété de prune four-wheeled carriage with collapsible hood (1870); voiture découverte à quatre roues, victoria a variety of domestic pigeon (1879); variété de pigeon domestique, Victoria

Victoria Monk (RS, 19-20th)

(cf. Maria Monk 2)

Victoria regia (Bot., 1846)

water-maize; maïs d'eau, Victoria regia

VIOLET

violet (Bot., 14th)

a flower of the genus Viola; fleur de l'espèce Viola, violette to play the shrinking violet to affect an excessive modesty; affecter une modestie exagérée, faire sa violette

VITUS

St. Vitus's dance

(cf. St Guy's dance)

WALTER

Sir Walter Scott (RS, 1857, ob.) pot (of beer); chope (de bière)

WILFRED

Wilfred (1865-1910)

teetotaller; abstinent anti-alcoolique

WILKIE

Wilkie Bards (RS, 20th)

playing cards; cartes à jouer

WILLIAM

William

any banknote (1887, ob.); billet de banque quelconque

weak tea (1923); thé très léger, café de sainte Claire

William pear (Bot., 1814)

a variety of pear; variété de poire, bon-chrétien William

sweet-William (Bot., 1562)

a species of pink; sorte d'œillet

WILLIE

Willie

(child's) penis (1905); pénis (d'enfant)

cf. Louie 2 (US, 1920)

cf. Fanny Adams 1 (US, 1926)

cf. Mary Ann 1 (US, 1930)

cf. jimmy bag

John Willie (NC, 1910)

(cf. Abraham)

painted Willie(US, NC, 20th)

(cf. Mary Ann 1)

pee-Willie, piss-Willie (1925)

cf. Ethel 2

cf. Jack Straw 2

Uncle Willie (RS, 1870)

silly (adj.); niais, stupide

weary Willie

long-range shell (Mil., 1915-18); obus à longue portée

cf. Lawrence (1935)

WILLY

Willy-boy (US, NC, 1914)

(cf. Molly 2)

Willy Lee (RS, 1925)

(cf. Jenny Lea 2)

pee-Willy (1925)

(cf. Ethel 2)

silly Willy (17-20th)

(cf. silly Billy) 